Renata Lopes Rosa

Análise da associação dos protocolos de roteamento AODV e DSR com o algoritmo Gossip, Sistema de Quorum e com um novo algoritmo de economia de energia, PWSave

Dissertação apresentada à Escola Politécnica da Universidade de São Paulo para obtenção do Título de Mestre em Engenharia Elétrica. 
Renata Lopes Rosa

\title{
Análise da associação dos protocolos de roteamento AODV e DSR com o algoritmo Gossip, Sistema de Quorum e com um novo algoritmo de economia de energia, PWSave
}

\author{
Dissertação apresentada à Escola \\ Politécnica da Universidade de São \\ Paulo para obtenção do Título de \\ Mestre em Engenharia Elétrica. \\ Área de concentração: \\ Sistemas Eletrônicos \\ Orientador: \\ Prof. Dr. José Roberto de \\ Almeida Amazonas
}




\section{Ficha Catalográfica}

Rosa, Renata Lopes

Análise da associação dos protocolos de roteamento AODV e DSR com o algoritmo Gossip, Sistema de Quorum e com um novo algoritmo de economia de energia, PWSave. São Paulo, 2009. 124 p.

Dissertação (Mestrado) - Escola Politécnica da Universidade de São Paulo. Departamento de Telecomunicações e Controle.

1. Gossip. 2. Sistema de Quorum. 3. Tolerância a falha. 4. Rede ad hoc. 5. Protocolo de Roteamento. I. Universidade de São Paulo. Escola Politécnica. Departamento de Telecomunicações e Controle. II. Título. 
aos meus pais Lourdes e Samuel 


\section{Agradecimentos}

À Sabedoria Infinita (Deus), por ter me guiado ao longo dessa caminhada.

Aos meus pais e irmã pela paciência, compreensão e amor.

Ao meu namorado pelo incentivo e amor.

Ao INFOFAU, amigos do trabalho.

Ao meu orientador, Prof. Dr. Amazonas, pelo grande aprendizado.

A todos os demais amigos que me apoiaram e ajudaram na realização deste trabalho. 


\section{Resumo}

Este trabalho estuda a implementação do sistema de Quorum associado ao algoritmo epidêmico Gossip, a implementação de um novo algoritmo de economia de energia - o PWSave - e o protocolo de roteamento AODV em um cenário com e sem falhas de uma rede ad hoc com mobilidade.

Optou-se por implementar este trabalho em um ambiente de simulação, dado que a modelagem matemática da associação do Gossip, Quorum e PWSave com os 80 nós - quantidade de nós escolhida para o ambiente de simulação - apresentaria maior complexidade e demora ao abranger todas as variáveis de ambiente desse conjunto de soluções para cada nó presente na rede. A rotina de programação - com o uso de loops para os trabalhos repetitivos - presente no ambiente de simulação permite que os experimentos sejam efetuados mais rapidamente e com menor probabilidade de erros.

Os estudos [1], [2] demonstraram, respectivamente, que soluções abrangendo o algoritmo epidêmico Gossip e o sistema de compartilhamento de dados Quorum apresentam resultados favoráveis para uma rede ad hoc com alta mobilidade.

Em [1] é apresentado um cenário muito próximo ao implementado neste trabalho, com a utilização do algoritmo Gossip ao protocolo de roteamento Ad-Hoc On-Demand Distance Vector (AODV). Os parâmetros analisados foram os mesmos, a saber: routes requests (RREQ), perda de pacote, vazão e latência. Os resultados do cenário simulado mostram uma diminuição no número de RREQs em uma rede ad hoc, e os demais parâmetros, medidos no ambiente de simulação, são pouco afetados.

De acordo com [2] constata-se que há um aumento da resiliência e da vazão da rede e uma menor sobrecarga causada pela distribuição da informação na rede ad hoc pelo sistema de Quorum.

A associação do algoritmo Gossip com o sistema de Quorum resultou em uma diminuição considerável de RREQs e perda de pacotes, mas o parâmetro de consumo de energia, que deve ser um fator importante em uma rede ad hoc e/ou uma rede sensor, não apresentou nenhuma melhora. Portanto, foi implementada uma solução adicional ao Gossip e ao Quorum, com o desenvolvimento de um novo algoritmo de economia de energia denominado de PWSave, no simulador Glomosim com o protocolo de roteamento AODV. O PWSave é responsável pelo adormecimento dos nós da rede que não estejam processando informações, ou seja, os nós, no momento do adormecimento, não poderão trocar dados ou auxiliar na formação de rotas da rede.

O PWSave associado ao Gossip e ao sistema de Quorum apresenta resultados que refletem uma diminuição no consumo de energia próxima a $10 \%$ em comparação com a solução da associação do Gossip com o sistema de Quorum sem a implementação de PWSave. 
Os resultados da simulação mostram que a associação de Gossip, Quorum e PWSave acarreta uma redução no número de RREQs e na taxa de perda de pacotes sem degradar muito características de fluxo e latência, além de propiciar uma considerável economia no consumo de energia. 


\section{Abstract}

This work studies the implementation of the Quorum system associated with the Gossip algorithm, the implementation of a new power saving algorithm - the $P W S a v e$ - and the routing protocol AODV in a scenario with and without failures of an ad hoc network with mobility.

It has been chosen to implement this work in an environment of simulation, because the mathematical modeling of the association of Gossip, Quorum and PWSave with 80 nodes - number of nodes that has been chosen for the simulation environment - would present a higher complexity and delay to address all environment variables of the solutions set for each node present in the network. The programming routine - with the use of loops for the repetitive works - present in the simulation environment allows the experiments to be performed faster and with less probability of errors.

The studies [1], [2] have shown, respectively, that solutions covering the Gossip epidemic algorithm and the system for sharing data Quorum show favorable results for an ad hoc network with high mobility.

In [1] is presented a scenario very close to that implemented in this work, using the Gossip algorithm associated to the routing protocol Ad-Hoc On-Demand Distance Vector (AODV). The parameters analyzed were the same: routes requests (RREQ), packet loss, throughput and latency. The simulated scenario results show a decrease in the number of RREQs in an ad hoc network, and the other parameters, measured in the simulation environment, are little affected.

According to [2] it is noted that there is an increase in the resilience and throughput of the network and a lower overload caused by the distribution of the information in the ad hoc network by the Quorum system.

The association of the Gossip algorithm with the Quorum system resulted in a considerable decrease of RREQs and packet loss, but the parameter of energy consumption, which is an important factor in an ad hoc network and/or a sensor network, shows no improvement. Therefore, an additional solution was associated to the Gossip and to the Quorum, with the development of a new power saving algorithm named PWSave, in the simulator Glomosim with the routing protocol AODV. The PWSave is responsable for the sleeping state of the network nodes when they are not processing information: the nodes at the time of sleep cannot exchange data or assist in the building of network routes.

The PWSave associated with the Gossip and Quorum system provides a decrease of the energy consumption close to $10 \%$ compared to the association solution of the Gossip with the Quorum system without the PWSave implementation.

The results of simulation show that the association of the Gossip, Quorum and PWSave produces a reduction in the number of RREQs and in the rate of 
packets loss without degrading much the throughput and latency characteristics, providing a considerable energy consumption economy. 


\title{
Sumário
}

\section{Lista de Figuras}

\author{
Lista de Tabelas
}

\section{Lista de Abreviaturas}

1 Introdução 1

1.1 Contexto e Motivação . . . . . . . . . . . . . . . . . . . 1

1.2 Descrição do Mapa Conceitual . . . . . . . . . . . . . . . . 5

1.3 Objetivo ............................. 6

1.4 Organização do Trabalho . . . . . . . . . . . . . . . . . . 7

2 Revisão Teórica $\quad 8$

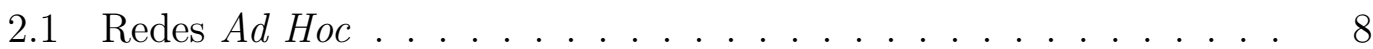

2.2 Protocolos de Roteamento . . . . . . . . . . . . . . . . . . . . . 9

2.2.1 Características Importantes de um Protocolo de Roteamento

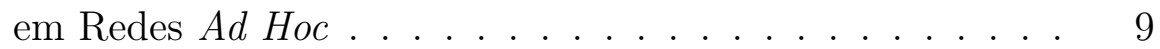

2.2.2 Classificação dos Protocolos de Roteamento . . . . . . . . 11

2.2.3 Protocolos de Roteamento On-Demand . . . . . . . . . . 12

2.3 Algoritmo Gossip . . . . . . . . . . . . . . . . . . . . 16

2.4 Sistema de Quorum . . . . . . . . . . . . . . . . . 20

2.5 Parâmetros de Desempenho . . . . . . . . . . . . . . . . . 21

3 Desenvolvimento de Novo Algoritmo para Economia de Energia em Redes Ad-hoc

3.1 Algoritmos Power Aware já existentes . . . . . . . . . . . . . 23 
3.2 Algoritmo PWSave . . . . . . . . . . . . . . . . . . 24

3.2.1 Fase de Associação dos Nós ao Sistema Servidor do Quorum 25

3.2.2 Fase de Adormecimento dos Nós que não pertencem ao conjunto do servidor do Quorum . . . . . . . . . . . 27

3.2 .3 Validação do Algoritmo PWSave . . . . . . . . . . . 29

4 Resultados Experimentais $\quad 34$

4.1 Simulador . . . . . . . . . . . . . . . . . . . . . 34

4.2 Cenário de Simulação . . . . . . . . . . . . . . . . . . . 35

4.2.1 Cenário de Implementação do algoritmo Gossip e Sistema de Quorum, sem falhas .............. . 35

4.2 .2 Cenário de Implementação de Falhas . . . . . . . . . . 36

4.2.3 Cenário de Implementação do algoritmo PWSave . . . . . 38

4.3 Resultados de Simulação . . . . . . . . . . . . . . . 40

4.3.1 Resultados da Implementação do algoritmo Gossip nos protocolos de roteamento Dynamic Source Routing (DSR) e Ad-Hoc On-Demand Distance-Vector (AODV) . . . . . . 41

4.3.2 Resultados da Implementação do algoritmo Gossip e Sistema de Quorum . . . . . . . . . . . . . . 45

4.3.3 Resultados da Implementação de Falhas . . . . . . . . 47

4.3.4 Resultados da Implementação do algoritmo PWSave . . . 53

5 Conclusão $\quad 61$

5.1 Sumário e Contribuições . . . . . . . . . . . . . . . . . 61

5.2 Sugestões para Trabalhos Futuros . . . . . . . . . . . . 62

$\begin{array}{ll}\text { Referências } & 63\end{array}$

$\begin{array}{ll}\text { Anexo A } & 66\end{array}$

Mapa Conceitual . . . . . . . . . . . . . . . . . 66 
Código original do AODV no Glomosim adaptado para esta dissertação 86 aodv.h .......................... 86

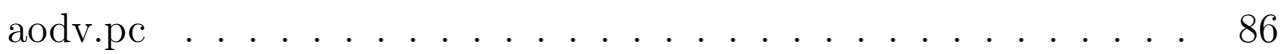

Código original do DSR no Glomosim adaptado para esta dissertação 90

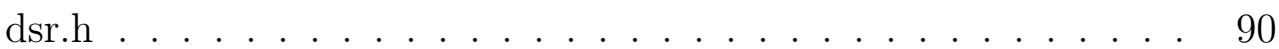

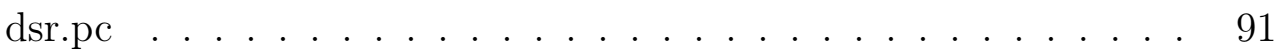

Anexo D - Código fonte do sistema de Quorum 96

Código original do AODV adaptado para esta dissertação . . . . . . . . 96

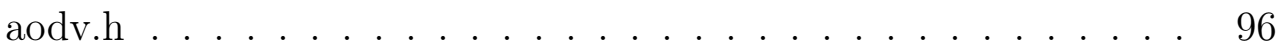
aodv.pc ................................. 97

$\begin{array}{ll}\text { Anexo E - Código fonte do sistema de Falhas } & 103\end{array}$

Código original do AODV adaptado para esta dissertação - Cenário A . 103

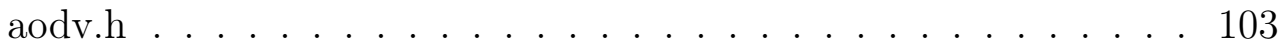

Código original do AODV adaptado para esta dissertação - Cenário B . 107 aodv.h ......................... 107 aodv.pc . . . . . . . . . . . . . . . 107

\section{Anexo F - Código fonte do algoritmo PWSave 112}

Código original do AODV adaptado para esta dissertação . . . . . . . . 112 aodv.h ........................... 112 aodv.pc . . . . . . . . . . . . . . . . 112 


\section{Lista de Figuras}

2.1 Problema do terminal oculto. . . . . . . . . . . . . . . 10

2.2 Problema do terminal exposto. . . . . . . . . . . . . . . . . . 10

2.3 Categorias de protocolos de roteamento [3] . . . . . . . . . . . 11

2.4 Mensagens RREQ e RREP no AODV. . . . . . . . . . . . . . . 14

2.5 Passos da disseminação da informação no Gossip. . . . . . . . . 19

2.6 Sistema de leitura e escrita no Quorum . . . . . . . . . . 21

3.1 Diagrama de estados da fase de associação do algoritmo PWSave implementado no lado do servidor do Quorum. . . . . . . . . . 25

3.2 Diagrama de tempo de associação do nó com o servidor do Quorum. 26

3.3 Diagrama de estados da fase de associação do algoritmo PWSave implementado no lado do nó não pertencente ao conjunto do servidor do Quorum. .................... . 27

3.4 Diagrama de estados da fase de adormecimento do algoritmo PWSave implementado no lado do servidor do Quorum. . . . . . . . . . . . 28

3.5 Diagrama de estados da fase de adormecimento do algoritmo PWSave implementado no lado do nó não pertencente ao conjunto do servidor do Quorum. . . . . . . . . . . . . . . . . 29

3.6 Cenário inicial de testes de 6 nós . . . . . . . . . . . . . . . . 30

3.7 Momento de associação do nó 1 ao nó 0 . . . . . . . . . . . . 30

3.8 Mobilidade dos nós . . . . . . . . . . . . . . . . . . . . . 31

3.9 Nó 3 recebendo pacotes no nó $1 \ldots$. . . . . . . . . . . . . 31

3.10 Estado de dorme/acorda do nó 2 e envio de pacotes do nó 1 para

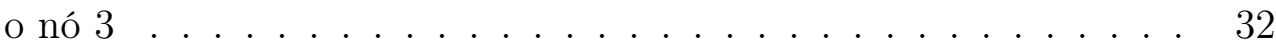

3.11 Estado de dorme/acorda do nó 3 e envio de pacotes do nó 1 para o nó $3 \ldots \ldots \ldots \ldots \ldots \ldots \ldots$ 
3.12 Estado de dorme/acorda do nó 4 e envio de pacotes do nó 1 ao nó 333

4.1 Histograma das simulações para o parâmetro RREQ do DSR, com e sem Gossip, com um tempo de pausa entre os deslocamentos de 50 segundos. . . . . . . . . . . . . . . . . . 36

4.2 Posição dos nós servidores do Quorum . . . . . . . . . . . . . 38

4.3 Perda de pacotes nos diferentes tempos de adormecimento dos nós 39

4.4 Consumo de energia nos diferentes tempos de adormecimento dos

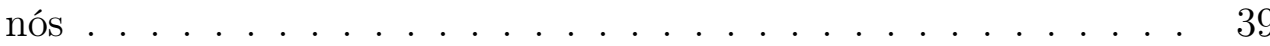

4.5 Distribuição Normal do DSR com Gossip com tempo de pausa de mobilidade de 50 segundos. . . . . . . . . . . . . . . .

4.6 Mensagens RREQs para AODV e DSR com e sem a aplicação do algoritmo Gossip em diferentes tempos de pausa. . . . . . . . .

4.7 Vazão para AODV e DSR com e sem a aplicação do algoritmo Gossip em diferentes tempos de pausa . . . . . . . . . .

4.8 Latência para AODV e DSR com e sem a aplicação do algoritmo Gossip em diferentes tempos de pausa . . . . . . . . . .

4.9 Perda de pacotes para AODV e DSR com e sem a aplicação do algoritmo Gossip em diferentes tempos de pausa . . . . . . . . .

4.10 Consumo de energia para AODV e DSR com e sem a aplicação do algoritmo Gossip em diferentes tempos de pausa . . . . . . . . .

4.11 Perda de pacotes para AODV com algoritmo Gossip e sistema de Quorum em diferentes tempos de pausa . . . . . . . . . .

4.12 Perda de pacotes para DSR com algoritmo Gossip e sistema de Quorum em diferentes tempos de pausa . . . . . . . . . .

4.13 Distribuição normal - AODV + Gossip + Quorum com tempo de pausa de 150 seg e falha de $30 \%$.

4.14 Histograma - AODV + Gossip + Quorum com tempo de pausa de 150 segundos e falha de $30 \%$. . . . . . . . . . . . . . . . 47

4.15 Latência - Cenário A . . . . . . . . . . . . . . . . . . . . . . 48

4.16 Latência - Cenário B . . . . . . . . . . . . . . . . . . . . 49

4.17 Vazão - Cenário A . . . . . . . . . . . . . . . . 50 
4.18 Vazão - Cenário B . . . . . . . . . . . . . . . . . . . 51

4.19 RREQ - Cenário A . . . . . . . . . . . . . 51

4.20 RREQ - Cenário B . . . . . . . . . . . . . . . . . 52

4.21 Consumo de Energia - Cenário A . . . . . . . . . . . . . . . 52

4.22 Consumo de Energia - Cenário B . . . . . . . . . . . . . . 53

4.23 Perda de pacotes - Cenário A . . . . . . . . . . . . 54

4.24 Perda de pacotes - Cenário B . . . . . . . . . . . . . 54

4.25 Perda de pacotes . . . . . . . . . . . . . . . 55

4.26 Índices de mérito de perda de pacotes a 7,5\% de falha com porcentagens de 50, 75 e 25\% no índice de mérito - Cenário B . . . . 56

4.27 Latência . . . . . . . . . . . . . . . . . . . . 56 56

4.28 Vazão . . . . . . . . . . . . . . . . . . . . . . . 5 57

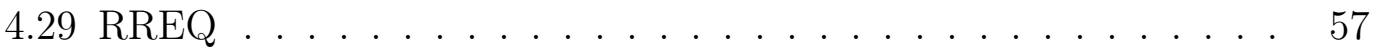

4.30 Índices de mérito para os parâmetros a 7,5\% de falha - Cenário B 58

4.31 Índices de mérito de perda de pacotes a 15\% de falha - Cenário B 59

4.32 Índices de mérito de perda de pacotes a 22,5\% de falha - Cenário B 59

4.33 Índices de mérito de perda de pacotes a 30\% de falha - Cenário B 60

4.34 Consumo de Energia . . . . . . . . . . . . . . . 60 


\section{Lista de Tabelas}

1.1 Caracterização das falhas segundo suas causas . . . . . . . . . . 2

2.1 Diferenças entre AODV e DSR . . . . . . . . . . . . . . 15

4.1 Falhas do Cenário A . . . . . . . . . . . . . . . . 38

4.2 Valores médios e desvios padrões para latência do Cenário A em

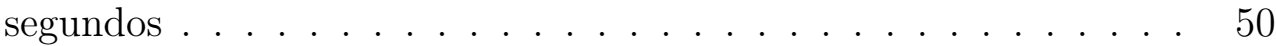

5.1 Valores médios e desvios padrões para latência do Cenário B em

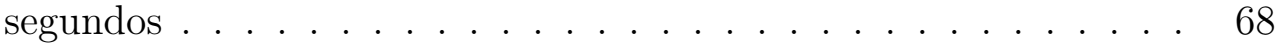

5.2 Valores médios e desvios padrões para vazão do Cenário A em bits $/$ seg. . . . . . . . . . . . . . . . . .

5.3 Valores médios e desvios padrões para vazão do Cenário B em bits $/$ seg. . . . . . . . . . . . . . . . . . 70

5.4 Valores médios e desvios padrões para RREQ do Cenário A . . . . 71

5.5 Valores médios e desvios padrões para RREQ do Cenário B . . . . 72

5.6 Valores médios e desvios padrões para o consumo de energia do Cenário A em mWhr . . . . . . . . . . . . . . . . . . 73

5.7 Valores médios e desvios padrões para o consumo de energia do Cenário B em mWhr . . . . . . . . . . . . . . . . . . . . . . 74

5.8 Valores médios e desvios padrões para a taxa de perda de pacotes do Cenário A em \% . . . . . . . . . . . . . . . 75

5.9 Valores médios e desvios padrões para a taxa de perda de pacotes do Cenário B em \% . . . . . . . . . . . . . . . . 76

5.10 Valores médios e desvios padrões para a taxa de perda de pacotes do Cenário A em \% . . . . . . . . . . . . . . . 77

5.11 Valores médios e desvios padrões para a taxa de perda de pacotes do Cenário B em \% . . . . . . . . . . . . . . . . . . . 78 
5.12 Valores médios e desvios padrões para latência do Cenário A em segundos . . . . . . . . . . . . . . . . . . . . . 79

5.13 Valores médios e desvios padrões para latência do Cenário B em

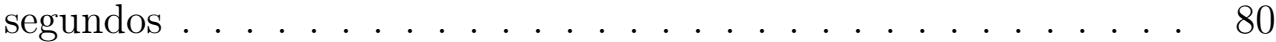

5.14 Valores médios e desvios padrões para vazão do Cenário A em bits $/$ seg. . . . . . . . . . . . . . . . . . . . 81

5.15 Valores médios e desvios padrões para vazão do Cenário B em bits/seg. . . . . . . . . . . . . . . . . . 82

5.16 Valores médios e desvios padrões para RREQ do Cenário A . . . . 83

5.17 Valores médios e desvios padrões para RREQ do Cenário B . . . . 84

5.18 Valores médios e desvios padrões para consumo de energia do Cenário A em mWhr . . . . . . . . . . . . . . . . . . 85 


\section{Lista de Abreviaturas}

ACK Acknowledgement

AODV Ad-Hoc On-Demand Distance-Vector

BcastID Identificador de broadcast

CBR Constant Bit Rate

CSMA/CD Carrier Sense Multiple Access with Collision Detection

DestID Identificador do destino

DestSeqNum Número de seqüência do destino

DSR Dynamic Source Routing

GCC GNU C Compiler

I Corrente

IEEE Institute of Electrical and Electronics Engineers

MAC Media Access Control

P Potência

Q Quorum

QoS Quality of Service

Qr Quorum de Leitura

QS Quorum Server

Qw Quorum de Escrita

RREQ Route Request

RREP Route Reply

RRER Route Error 
Rx Recepção de Dados

SrcID Identificador fonte

SrcSeqNum Número de sequência da fonte

TTL Tempo de vida das mensagens

Tx Transmissão de Dados

V Tensão 


\section{Introdução}

Neste capítulo serão abordados a importância deste trabalho nos dias atuais, a descrição do mapa conceitual que determinou o tema deste trabalho, seu objetivo e a organização dos capítulos.

\subsection{Contexto e Motivação}

As redes ad hoc são redes não infra-estruturadas de transmissões de dados, formadas por dispositivos móveis capazes de trocar informações entre si de maneira eficaz e não posssuem uma administração central como nas redes infra-estruturadas de telefonia celular e nas redes locais sem fio com pontos de acesso.

Elas apresentam uma grande importância nos dias atuais, pois podem ser utilizadas, na forma de sensores, para auxiliar em diversas atividades, tais como: conhecimento da localização de rebanhos no pasto, determinação de temperaturas em estufas de plantações, geoprocessamento, aplicações militares, computação distribuída e colaborativa e auxílio em tráfego rodoviário e/ou ferroviário para diminuição de acidentes.

Em uma rede ad hoc cada dispositivo serve como roteador por possuir alcance limitado de transmissão.

Em uma rede convencional os pontos de acesso não apenas fornecem a comunicação entre os nós da rede, como também intermedeiam o tráfego com os pontos vizinhos de acesso. Os ambientes desprovidos de uma infra-estrutura prévia são os mais beneficiados com as rede móveis ad hoc.

O fato dos dispositivos ad hoc possuírem uma grande mobilidade faz com que seja necessária uma permanente adaptação e reconfiguração das rotas. Os dispositivos ad hoc também estão submetidos a danos que podem torná-los inoperantes. Por tais motivos, é necessário que as redes ad hoc sejam tolerantes a falhas, ou que, ao apresentar falhas, parâmetros importantes como perda de pacote, latên- 
cia, route requests, vazão e consumo de energia não sejam drasticamente afetados.

Portanto, o protocolo de roteamento é um fator muito importante a ser estudado nas redes ad hoc.

Em [4] são definidas algumas características de uma rede ad hoc móvel 802.11b como: o número de dispositivos alcançados, grau do nó, comprimento médio do trajeto, tempo de vida do enlace e tempo de vida de uma rota para servir como parâmetros para validação protocolos de rede novos ou já existentes.

Tabela 1.1: Caracterização das falhas segundo suas causas

\begin{tabular}{ccc}
\hline Causa da & Persistência & Extensão \\
falha & & \\
\hline Fenômenos atmosféricos & permanente & agrupadas \\
Fontes móveis de interferência & transiente & isolada \\
Desastres naturais & permanente & agrupadas \\
Quebra acidental & permanente & isolada \\
Bloqueio do processador & transiente & isolada \\
Ataques de interferência & permanente & agrupadas \\
Ataques de colisão & ambos & isolada \\
Ataques de sinkhole & ambos & isolada \\
\hline
\end{tabular}

A Tabela 1.1 classifica as causas de falhas que ocorrem nos dispositivos móveis quanto à persitência e extensão. As falhas podem ser momentâneas (transientes) ou permanentes, de acordo com o tempo de inatividade do dispositivo. As falhas ocorrem de maneira isolada, como no caso de ataques sinkhole ${ }^{1}$, ou em grupo.

As novas redes ad hoc devem ser projetadas para prover serviços contínuos e confiáveis. Em [5] é apresentado um serviço de detecção de falhas em uma rede de sensores de grande escala, baseado no algoritmo Gossip e em um sistema heartbeat, responsável por enviar periodicamente mensagens de monitoramento aos dispositivos da rede notificando quais os dispositivos ainda continuam ativos.

Um sistema tolerante a falhas deve manter um desempenho adequado em relação ao atraso, à carga e ainda manter conectividade entre os nós no caso da perda de um ou mais caminhos entre um dispositivo falho e os seus vizinhos. As mensagens de atualização dos paths não devem sobrecarregar o sistema a ponto de torná-lo inoperante, devendo haver uma solução distribuída com o balanceamento dessas informações.

\footnotetext{
${ }^{1} \mathrm{O}$ ataque de sinkhole citado na Tabela 1.1 é um ataque em que todo o tráfego de informação se dirige para um determinado nó, inutilizando-o
} 
O algoritmo de características probabilísticas, Gossip, foi escolhido para este trabalho por ser tolerante a falhas, mantendo uma carga balanceada entre os dispositivos de rede e por apresentar uma quantidade menor de mensagens de atualização de rota na rede. Em [1] apresenta-se uma diminuição de 35\% de mensagens Route Request (RREQ) comparando-se com a técnica de flooding (informação repassada a todos os nós da rede), acarretando em uma menor sobrecarga da rede. Porém, no artigo referido, as análises do Gossip foram feitas somente com o protocolo AODV, não comparando com demais protocolos de roteamento.

Uma outra solução para o problema foi apresentada por [2] a partir do estudo das comunicações em grupo e do compartilhamento de dados, sistema de Quorum, e do algoritmo Gossip.

No entanto, a associação do algoritmo Gossip com o sistema de Quorum não aborda um parâmetro muito importante nos dias atuais, o consumo de energia.

O consumo de energia dos nós é um fator crítico na operação das redes ad hoc. Tipicamente, nós ad hoc são dispositivos sem fio alimentados por baterias cujas capacidades de armazenamento são limitadas por restrições de volume e peso dos equipamentos. Por isso, é importante reduzir o consumo de energia dos nós.

O problema da conservação de energia em redes ad hoc vem sendo abordado de diversas formas. Uma delas é utilizar a energia restante dos nós como métrica de roteamento, técnica que foi discutida inicialmente por [6]. O objetivo da energia como métrica de roteamento é evitar a utilização de uma mesma rota repetidas vezes, o que levaria ao rápido esgotamento da energia dos nós. O uso não repetitivo de uma mesma rota procura balancear os fluxos de dados, levando em consideração a energia restante nos nós da rede. O balanceamento de tráfego é mais simples em redes estáticas onde a topologia pode ser seguramente obtida, porém é mais complexo em redes móveis que possuem topologias dinâmicas.

Em [7] são apresentados algoritmos para balancear fluxos de dados com o objetivo de maximizar o tempo de vida dos nós da rede. De acordo com [8] propõe-se duas novas versões do protocolo Dynamic Source Routing que visam a maximização do tempo até que o primeiro nó da rede fique sem energia, porém é adicionado um determinado atraso na descoberta de rotas.

Em [9] é desenvolvido um algoritmo de roteamento capaz de alterar as rotas durante as conexões, protegendo nós próximos à exaustão.

Em [10] é analisada a melhor rota numa determinada topologia considerando 
a potência de transmissão dos nós variável. A abordagem de [6] busca o balanceamento do consumo de energia dos nós dividindo as conexões em dois segmentos, um de comunicação com múltiplos saltos e outro com comunicação direta.

Neste trabalho é analisado o consumo dos nós ad hoc com a utilização do algoritmo Gossip com o sistema de Quorum e, para o aproveitamento dessa associação que apresenta resultados satisfatórios em relação à perda de pacotes, vazão e routes requests, conclui-se que uma boa solução seria o adormecimento dos nós quando estes não estiverem recebendo e enviando dados e/ou auxiliando na construção de rotas. Decidiu-se, então, pela implementação de um novo algoritmo de economia de energia, denominado $P$ WSave, que apresentou resultados bastante satisfatórios na economia de energia juntamente com os bons resultados já obtidos nos demais parâmetros.

Associando os estudos do algoritmo Gossip, o sistema de Quorum e o algoritmo PWSave chegou-se ao tema deste trabalho, que será diferente de [2] por abranger mais de um protocolo de roteamento para comparações e validações de desempenho da rede e por estudar o parâmetro de consumo de energia. Diferenciase dos demais trabalhos citados por propor um algoritmo de economia de energia que não interfira no desempenho do conjunto de parâmetros (latência, vazão, perda de pacotes e RREQ), implicando em um menor consumo de energia em um cenário móvel, com o uso do protocolo AODV, e sem o uso do agendamento para adormecer os nós que seria um cenário mais limitado de uso.

A solução proposta neste trabalho possui aplicações em: redes de sensores sem fio na monitoração fisiológica de bovinos, onde há a necessidade de experimentar técnicas de coleta de dados, que causem menos perturbações no comportamento natural dos animais de interesse de zootecnia de precisão; redes de sensores sem fio presos a galhos de árvores formando um tipo de instrumento científico macroscópico capaz de registrar o microclima ao redor de determinadas áreas para fins de prevenção ao desmatamento, auxílio biológico e ecológico para compreender o comportamento da fauna e flora de determinada região, e auxílio na monitoração da qualidade do ar em áreas urbanas.

O tema deste trabalho foi escolhido utilizando uma ferramenta de construção de mapas conceituais chamada IHMC CMAP Tools que organiza as referências bibliográficas de uma maneira fácil e bem didática, auxiliando na escolha do tema, o que é explicado em detalhes em 1.2. Conforme visto no mapa conceitual, no Anexo $\mathrm{A}^{2}$, são apresentadas soluções relacionadas a: somente exclusão de dis-

\footnotetext{
${ }^{2}$ o mapa conceitual foi colocado em anexo devido ao tamanho usado para a impressão
} 
positivo, somente detecção de dispositivos vizinhos estudando a sobrecarga e/ou atraso da rede e/ou largura de banda, e que, por sua vez, propõem modificações ou criação de novos protocolos de atualização de rotas ou soluções distribuídas.

\subsection{Descrição do Mapa Conceitual}

A ferramenta de construção de mapas conceituais chamada IHMC CMAP Tools, como já foi mencionada, auxiliou na escolha do tema deste trabalho, mostrando-se útil na separação dos temas relacionados às redes móveis ad hoc.

Os conceitos aparecem dentro de caixas enquanto que as relações entre os conceitos são especificadas através de frases de ligação nos arcos que unem os conceitos. As frases de ligação têm funções estruturantes e exercem papel fundamental na representação de uma relação entre dois conceitos.

O mapa conceitual, mostrado no anexo $A$, tem início com as redes móveis ad hoc que foram escolhidas devido o seu crescente uso. Na seqüência são abordadas suas características de autoconfiguração, conectividade, mobilidade e tolerância a falha. A característica de tolerância a falha é de suma importância na sustentação de uma boa comunicação entre seus dispositivos. Os tipos de falhas são abordados e os vários problemas e soluções são citados com a utilização de referências bibliográficas compostas por artigos de revistas publicadas pelo Institute of Electrical and Electronics Engineers (IEEE).

O mapa aborda problemas e soluções relacionados a:

1. escolha dos novos líderes da rede ad hoc para servir como coordenador que auxilia na definição da ordem de processos no sistema;

2. soluções relacionadas a multicast como em [11], [12], [13], [14], [15] e [2]. Descoberta da localização geográfica dos nós de [16] e [17];

3. exclusões de nós abordando a parte algorítmica em [18] e [19];

4. protocolos de roteamento de Multipath [20] e [21];

5. problemas abordando Quality of Service (QoS) como [22] e [23];

6. redução de interferência de canais em [24]. 


\subsection{Objetivo}

O objetivo desta pesquisa é estudar como obter uma melhora dos principais parâmetros (RREQ, perda de pacote, vazão, latência e consumo de energia) em uma rede ad hoc móvel, com a utilização dos protocolos de roteamento AODV e DSR, associados com o algoritmo probabilístico Gossip e sistema de Quorum e a utilização do AODV com um novo algoritmo de economia de energia implementado, o PWSave. O trabalho proposto utiliza o simulador Glomosim, escolhido por já ter sido utilizado em [1], uma importante referência para análise dos resultados de simulação.

Neste trabalho, será apresentado como as rede móveis ad hoc se comportam diante de um ambiente com e sem falhas e propor melhorias para torná-las mais robustas. Para esse fim, serão abordados os protocolos de roteamento mais utilizados, um algoritmo probabilístico de disseminação de informação, um sistema de replicação de dados e um algoritmo de economia de energia.

As principais contribuições desta pesquisa são:

1. Análise, em um ambiente sem falhas, da associação do algoritmo probabilístico de disseminação de informação, chamado Gossip, com os protocolos de roteamento AODV e DSR. O desempenho da rede será avaliado pela medida de entrega de pacotes, RREQ, vazão, latência e consumo de energia.

2. Implementação específica de um sistema de Quorum, para uma rede de sensores ad hoc sem fio.

3. Análise, em um ambiente sem falhas, da associação do Gossip com os protocolos AODV e DSR com o sistema de replicação de dados, nomeado de sistema de Quorum. O desempenho da rede será avaliado pela medida dos mesmos parâmetros já anteriormente citados.

4. Análise, em um ambiente com falhas, da associação do Gossip com o protocolo AODV juntamente com o sistema de Quorum. O desempenho da rede será avaliado pela medida dos mesmo parâmetros já anteriormente citados.

5. Implementação de um novo algoritmo de economia de energia, (PWSave) e análise do seu impacto, com o algoritmo Gossip e sistema de Quorum utilizando o protocolo AODV, no consumo de energia em um ambiente com e sem falhas e comparação com protocolos power aware já existentes. 


\subsection{Organização do Trabalho}

Neste Capítulo, apresenta-se uma seção introdutória expondo o contexto e motivação para associação e implementação do algoritmo Gossip, sistema de Quorum e o algoritmo PWSave em redes ad hoc, a descrição do Mapa conceitual e os objetivos desta pesquisa.

O Capítulo 2 constitui uma revisão teórica dos tópicos: protocolos de roteamento (AODV e DSR), algoritmo Gossip, sistema de Quorum e parâmetros de desempenho medidos no ambiente de simulação.

No Capítulo 3 é abordado a descrição da implementação do novo algoritmo de economia de energia, PWSave, pelos diagramas de estado.

Em seguida, apresentam-se os resultados experimentais no Capítulo 4, dividido em três subseções que discutem o simulador, os cenários implementados e os resultados obtidos em relação aos cenários de simulação.

Conclusões e sugestões para trabalhos futuros são discutidos no Capítulo 5.

No Anexo A é apresentado o mapa conceitual que auxiliou na escolha do tema deste trabalho, no Anexo B são apresentadas as tabelas referentes aos resultados das simulações efetuadas e os demais anexos (C, D, E e F) apresentam os códigos implementados, na linguagem Parsec, do algoritmo Gossip, sistema de Quorum, sistema de falhas no cenário A e B e do algoritmo PWSave, respectivamente. 


\section{Revisão Teórica}

Neste capítulo será feita a revisão teórica dos principais tópicos abordados neste trabalho.

\subsection{Redes $\mathrm{Ad}$ Hoc}

O termo ad hoc é, entendido como algo que é criado ou usado para um problema específico ou imediato. Contudo, ad hoc em termos de redes ad hoc sem fio significa mais que isso. Em uma rede ad hoc não há uma topologia pré-determinada e nem um controle centralizado. Redes ad hoc não requerem uma infra-estrutura tal como um backbone, ou pontos de acesso configurados antecipadamente. Os nós ou nodos se comunicam sem o uso de uma conexão física entre eles criando uma rede on the fly, na qual, alguns dos dispositivos da rede fazem parte desta. De fato, apenas durante a sessão de comunicação ou, no caso de dispositivos móveis ou portáteis, no momento em que estão a uma certa proximidade dos demais nós da rede e é necessário a troca de informações entre eles.

Uma rede ad hoc móvel (MANET) é um conjunto de nós móveis formando redes dinâmicas autônomas e independentes de qualquer infra-estrutura. Os nós sendo móveis, a topologia da rede pode mudar rapidamente e de forma inesperada a qualquer instante.

Devido ao alcance dos nós de transmissão das redes sem fio, múltiplos saltos (hops) podem ser necessários para efetuar a troca de dados entre os nós da rede, daí o termo rede multi-hop. Nessa rede, cada nó atua tanto como roteador quanto como um host, participando da descoberta e manutenção de rotas para outros nós. 


\subsection{Protocolos de Roteamento}

Em uma rede ad hoc móvel, as rotas entre os nós estão sempre sendo refeitas, portanto, o protocolo de roteamento é muito importante para a interconexão dos nós.

Protocolos de roteamento, já existentes para as redes cabeadas, não podem ser utilizados para as redes ad hoc, pois estas apresentam uma estrutura diferente, ou seja, as redes ad hoc possuem: uma topologia mais dinâmica, não infra-estruturada, largura de banda mais restrita e limitação do tempo de vida da bateria.

\subsubsection{Características Importantes de um Protocolo de Rotea- mento em Redes Ad Hoc}

Os principais desafios ao se projetar um protocolo de roteamento de uma rede ad hoc, segundo [3], estão relacionados à: mobilidade dos nós, contenção de recursos, transmissão broadcast e problemas do terminal oculto e exposto.

Os problemas de mobilidade ocorrem devido à rede ad hoc ser altamente dinâmica.

Na utilização de recursos, a largura de banda de uma rede ad hoc é menor se for comparada à rede cabeada porque possui a limitação da banda de rádio e constantemente, existem as trocas de mensagens de atualização de rotas ocasionando sobrecarga na rede, havendo o maior consumo da largura de banda.

Os canais de rádio possuem características variáveis no tempo em termos de capacidade do enlace e de probabilidade de erro de bit do enlace. Além disso, nas redes ad hoc o protocolo de roteamento tem que interagir com a camada Media Access Control (MAC) para encontrar rotas alternativas por meio de um enlace de melhor qualidade. As transmissões neste tipo de rede resultam em colisões de dados e de pacotes de controle. Isto pode ocorrer devido a dois problemas: terminal oculto e terminal exposto. A Figura 2.1 ilustra o problema do terminal oculto. Neste caso, dois (ou mais) transmissores estão afastados e não conseguem detectar a colisão. O receptor comum recebe os sinais simultaneamente e é capaz de filtrar apenas 1 sinal. No caso da Figura 2.1, o terminal A está escondido do terminal C e suas transmissões colidem sobre o terminal B. A Figura 2.2 ilustra o problema do terminal exposto, onde B transmite para A e C quer enviar mensagens para D. Utilizando Carrier Sense Multiple Access with Collision De- 
tection (CSMA/CD), C escuta o meio ocupado, então C espera. Mas A está fora do alcance de C, então a espera de C é desnecessária e dessa forma, C está exposto a B.

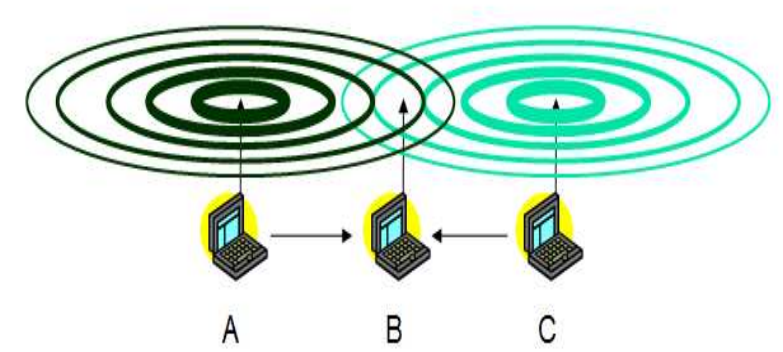

Figura 2.1: Problema do terminal oculto.

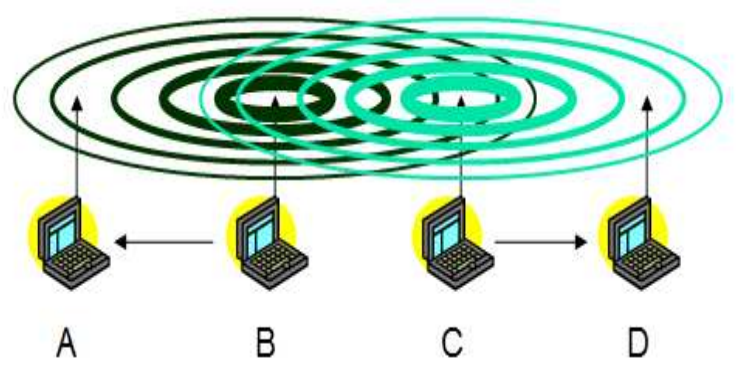

Figura 2.2: Problema do terminal exposto.

No problema da escassez de recursos incluem-se: tempo de vida da bateria e capacidade de processamento do nó.

Assim, conclui-se que um protocolo de roteamento para uma rede ad hoc deve:

1. Ser distribuído, pois o roteamento centralizado envolve um overhead de controle alto e não possui a característica de ser escalável. Roteamentos distribuídos são mais tolerantes a falhas.

2. Deve ser adaptativo, devido à mudança constante de topologia.

3. A manutenção de uma rota deve envolver o menor número possível de nós, com um tempo mínimo para efetuar as configurações.

4. O número de colisões de pacotes deve ser o mínimo possível, limitando o número de broadcasts na rede.

5. A procura das rotas deve convergir e isso deve ser rápido.

6. Deve economizar recursos como largura de banda, processamento, memória e bateria. 
7. Cada dispositivo deve armazenar informações das topologias locais estáveis e não quando ainda estiverem em fase de mudança de localização.

8. Deve ser capaz de manipular parâmetros de QoS.

\subsubsection{Classificação dos Protocolos de Roteamento}

Os protocolos de roteamento são divididos nas categorias ilustradas na Figura 2.3 e detalhadas a seguir:

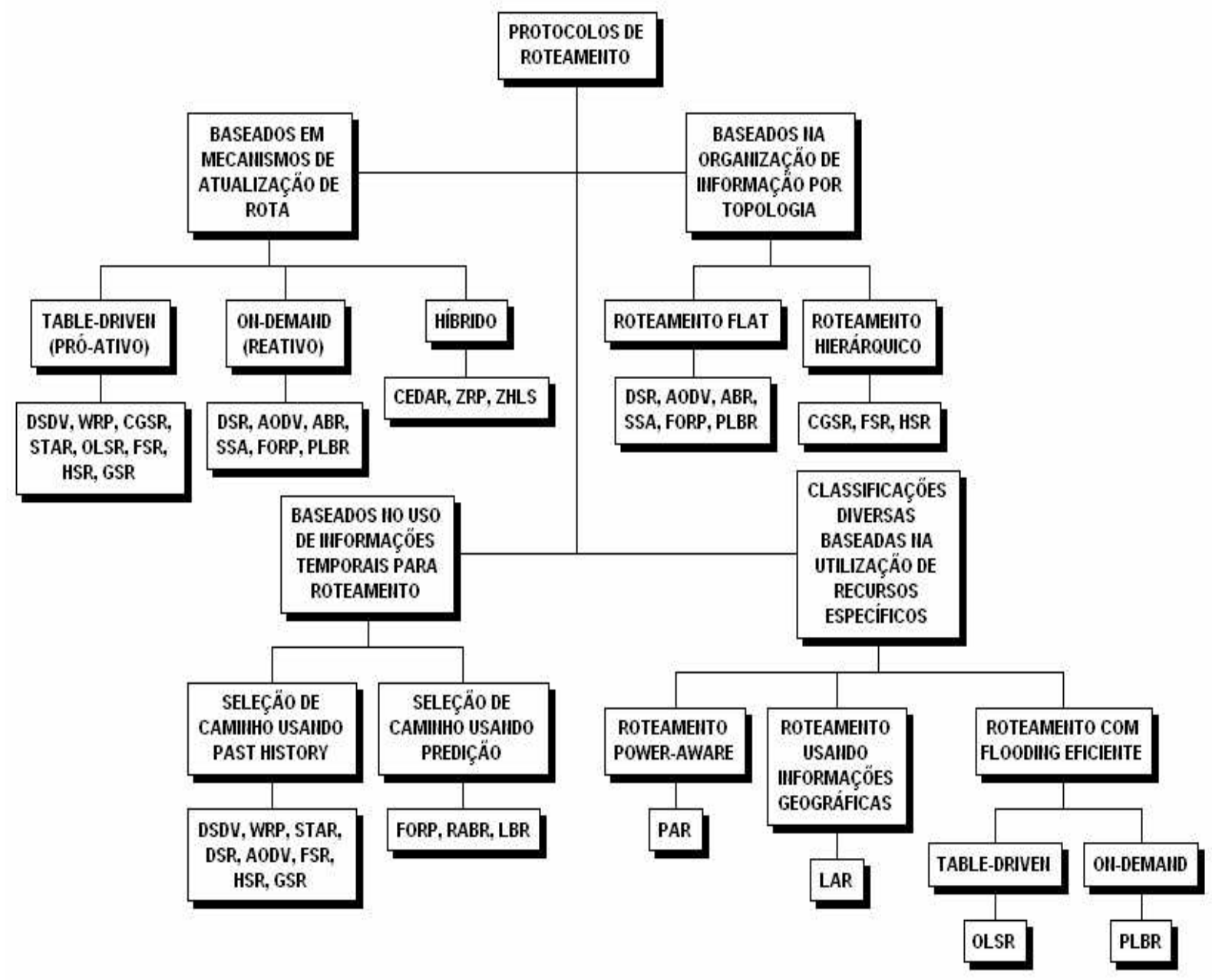

Figura 2.3: Categorias de protocolos de roteamento [3].

- Baseados em mecanismos de atualização de informações:

- Pró-ativos (ou table-driven): cada nó mantém informações atualizadas sobre a topologia da rede, todos os nós possuem completo conhecimento dos caminhos para todos os outros nós, por meio da troca regular de informações de roteamento. Estas informações são, geralmente, difundidas utilizando flooding por toda a rede. Sempre que um nó precisar de um caminho até o destino, é utilizado um algoritmo 
apropriado para determinação de rota, com base nas informações de topologia ou no melhor caminho mantido pelo próprio nó;

- Reativos (ou on-demand): não trocam informações de roteamento periodicamente, mas sim obtêm o caminho, quando necessário, por meio do processo de descoberta de rotas entre os nós de interesse;

- Híbridos: combinam características das duas categorias anteriores: pró-ativos e reativos.

- Baseados no uso de informações temporais:

- Usando informações temporais passadas: usa informações sobre o estado passado do enlace na hora de tomar decisões sobre o roteamento;

- Usando informações temporais futuras: possui uma expectativa futura sobre o estado do enlace tomando decisões aproximadas do roteamento.

- Baseados na topologia da rede:

- Topologia flat: usam um esquema de endereçamento similar ao utilizado nas redes IEEE 802.3 [25];

- Topologia hierárquica: fazem uso de uma hierarquia lógica e um esquema de endereçamento associado.

- Baseados na utilização de recursos específicos:

- Power-aware: objetivam minimizar o consumo de energia de um nó ou de toda a rede;

- Segundo informações geográficas: tendem a melhorar o desempenho de roteamento e reduzir o overhead de controle, utilizando informações geográficas da rede.

\subsubsection{Protocolos de Roteamento On-Demand}

São os protocolos mais utilizados em redes ad hoc [26], [27], [28] e [29].

Protocolos de roteamento on-demand trocam informações de roteamento somente quando um determinado caminho é solicitado. 


\subsubsection{Protocolo Ad-Hoc On-Demand Distance-Vector (AODV)}

Este protocolo utiliza as seguintes variáveis:

- Número de sequência da fonte (SrcSeqNum): número de seqüência gerado pelo nó fonte;

- Número de seqüência do destino (DestSeqNum): número de seqüência crescente usado como forma de se verificar o quão recente é uma rota, pois o DestSeqNum com o maior valor é que representará a rota mais atual;

- Identificador fonte (SrcID): especifica o nó fonte que pretende enviar pacotes a um determinado nó da rede;

- Identificador do destino (DestID): especifica o nó destino que deverá receber pacotes de um determinado nó fonte;

- Identificador de broadcast (BcastID): quando vários broadcasts são enviados à rede, cada um recebe um identificador que é relacionado a uma determinada mensagem RREQ, podendo assim identificá-la. É incrementado a cada nova requisição de rota enviada.

- Tempo de vida das mensagens (TTL): contador que representa o tempo que se considera até que uma mensagem possa ser descartada;

- Número de hops: número de saltos necessários para se alcançar um determinado nó a partir de um nó origem.

O protocolo possui as seguintes mensagens:

- Mensagens HELLO: enviadas periodicamente para se confirmar a conectividade local entre vizinhos;

- RREQ: mensagens de requisição de rota enviadas a toda a rede para encontrar o nó destino, quando a rota já não é conhecida;

- Route Reply (RREP): resposta à requisição de rota especificando como chegar até o nó destino;

- Route Error (RERR): aviso de queda de enlace. Quando um enlace deixa de existir entre dois nós é enviado uma RERR. 
Quando um nó quer enviar um pacote a outro nó e ainda não possui uma rota determinada, o nó fonte envia um RouteRequest (contendo SrcID, DestID, SrcSeqNum, DestSeqNum, BcastID e TTL a todos os seus vizinhos por difusão. Caso um vizinho não seja o destino ou não possua uma rota válida para o destino, ele repassa a RREQ aos seus vizinhos . Este processo se repete até a requisição atingir o destino ou um nó que conheça uma rota para este, inundando a rede.

Um nó de destino, ao responder a RREQ, envia pelo caminho reverso uma mensagem RREP, que contém o endereço da fonte e do destino, o número de seqüência do destino, o contador de saltos (incrementado de uma unidade a cada salto) e seu TTL. Antes de enviar a RREP, o nó de destino atualiza o seu número de seqüência para o máximo entre o valor atual e o valor que constava na RREQ. Em cada nó atravessado no caminho reverso pela RREP é armazenado, em uma entrada referente ao destino, o próximo salto para alcançar o destino, ou seja, o endereço do vizinho de quem se recebeu a resposta e o número de seqüência do destino.

A movimentação de um nó ativo pode provocar a queda de um dado enlace que estava sendo utilizado. Nesta situação uma mensagem de erro (RERR) é enviada a todos os nós afetados, avisando sobre a queda do enlace. Dessa forma, cada nó por onde passa a RERR deve decidir se continua ou não a repassar esta mensagem, além de ter que atualizar a sua tabela de roteamento, registrando que os destinos especificados na mensagem estão inalcançáveis e atualizando seus números de seqüência.

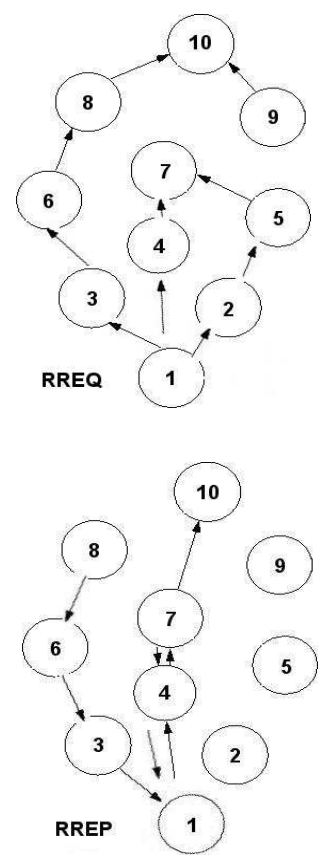

Figura 2.4: Mensagens RREQ e RREP no AODV. 
A Figura 2.4 ilustra as mensagens RREQ e RREP do AODV na rede. O broadcast de RREQ se inicia com o nó 1 enviando RREQ aos nós 2, 3 e 4, que por sua vez verificam se possuem a rota até o nó destino 10. Caso não possuam, eles enviam RREQ aos seus vizinhos diretamente conectados. Os nós 5, 6 e 7 são os vizinhos de 2, 3 e 4 respectivamente. Como o nó 7 possui uma rota até o nó destino 10, então ele envia uma RREP até o nó fonte. Caso outro nó possuísse também uma rota até o nó 10, este nó responderia com uma RREP ao nó fonte. Como, por exemplo, o nó 8.

\subsubsection{Protocolo Dynamic Source Routing (DSR)}

Utiliza as mesmas mensagens do protocolo AODV, possuindo um funcionamento similar a este, com a diferença que no DSR é usado um cache de rota que armazena todas as informações possíveis extraídas da rota, contida em um pacote de dados. No AODV somente a rota mais atual é armazenada, as demais são descartadas.

Ele foi projetado para consumir menos largura de banda, devido à eliminação de mensagens periódicas sobre o estado da rede para efeitos de atualização da sua tabela de rota. Esse protocolo se diferencia dos demais, on-demand, pelo fato dos nós não precisarem transmitir mensagens HELLO periódicas, com o propósito de notificar os vizinhos quando estão ativos.

Os protocolos AODV e DSR possuem algumas diferenças entre si, como as ilustradas na Tabela 2.1 e outras características que os distinguem como o fato de o DSR trabalhar com um número máximo de até 200 nós ou de 5 a 10 hops e o AODV trabalhar com um número superior a 200 nós.

Tabela 2.1: Diferenças entre AODV e DSR

\begin{tabular}{|c|c|c|c|c|}
\hline & $\begin{array}{l}\text { Suporta } \\
\text { múltiplos } \\
\text { caminhos }\end{array}$ & $\begin{array}{c}\text { Envia } \\
\text { mensagens } \\
\text { periódicas }\end{array}$ & $\begin{array}{c}\text { Nós } \\
\text { intermediários } \\
\text { necessitam } \\
\text { inteligência } \\
\text { para } \\
\text { encontrar } \\
\text { rota }\end{array}$ & $\begin{array}{c}\text { Informações } \\
\text { de roteamento } \\
\text { são trocadas } \\
\text { com mais de } \\
\text { um nível de } \\
\text { vizinho }\end{array}$ \\
\hline AODV & Sim & Sim & Sim & Não \\
\hline DSR & Não & Não & Não & Sim \\
\hline
\end{tabular}




\subsection{Algoritmo Gossip}

O objetivo deste protocolo é distribuir as informações para um grupo. O mecanismo do gossip consiste em que cada membro do grupo passa a informação periodicamente a um membro escolhido aleatoriamente. Uma de suas principais características é a escalabilidade e resilência a falhas de um processo ou mensagens perdidas.

Trata-se de um algoritmo multicast probabilístico, também chamado de algoritmo epidêmico. Compara-se o algoritmo a uma epidemia que é dificil de se erradicar (tolerante a falhas).

Nesta dissertação são empregados tanto o termo epidêmico como probabilístico para se referir ao algoritmo Gossip.

O algoritmo Gossip foi projetado inicialmente para melhorar o gerenciamento de base de dados replicados [30]. Posteriormente, foi utilizado em detecção de falhas e na detecção de memberships [14].

De acordo com [30], para um grupo de N máquinas, se em uma máquina tem início a disseminação da informação, esta máquina demora $O(\log N)$ rounds para alcançar cada máquina com uma determinada informação. Isso significa que o destino é alcançado rapidamente.

O algoritmo Gossip propaga as informações da mesma maneira que uma infecção viral, por isso é chamado de algoritmo epidêmico. Um nó repassa as informações somente a um determinado número de vizinhos, que é estabelecido por uma probabilidade especificada no algoritmo Gossip, evitando flooding da rede, a não ser que esta probabilidade seja de valor 1, que significa que $100 \%$ dos seus vizinhos devem receber as mensagens. Cada nó mantém apenas uma vista parcial de todo o sistema, a partir da qual efetua a seleção dos nós com os quais realizará a troca de informações.

A probabilidade de infecção dos membros de uma rede Gossip é expressa matematicamente por (2.1) [31]:

$$
p_{\text {connect }}=\frac{1}{e^{\frac{1}{e^{c}}}}
$$

O parâmetro $c$ se relaciona com o parâmetro $f$ (Fanout) através da equação $f=\log (n)+c$, utilizando o modelo contamina e morre (considera-se que cada 
nó contaminado só retransmite a mensagem uma vez), onde:

- $n$ : número de nós da rede;

- $f$ (Fanout): número de nós selecionados para receber a informação enviada pelo nó emissor. Valores altos de fanout garantem um nível de tolerância a falhas maior, porém há uma maior geração de tráfego redundante na rede.

Observa-se que quanto maior o valor de $c$, maior a probabilidade de infecção, mas por outro lado requer uma visão do sistema cada vez maior para cada nó $[31]$.

O fato de os emissores escolherem aleatoriamente a quem enviam as mensagens torna o Gossip tolerante a falhas e totalmente distribuído.

Os algoritmos Gossip podem ser classificados em:

- Gossip1 (p, k): efetua um broadcast para os primeiros k hops, permitindo que as primeiras mensagens sejam repassadas com uma probabilidade $\mathrm{p}=$ 1 (100\%). Depois de receber uma mensagem pela primeira vez, o nó a retransmite com $\mathrm{p}<1$.

- Gossip2 (p1, k, p2, n): efetua o repasse de mensagens para um nó, dependendo da sua localização com respeito ao nó fonte e o número de seus nós vizinhos (n).

Os parâmetros deste algoritmo serão explicados mais adiante.

- Gossip3 (p1, k, m): efetua um repasse de mensagens segundo um número suficiente de retransmissões (m). Se um nó decide não repassar uma mensagem e depois recebe menos que m cópias de mensagens, imediatamente faz um broadcast da mensagem.

A informação que é repassada de um nó para outro é chamada de Gossip.

Neste trabalho é utilizado o algoritmo Gossip2, que possui a vantagem de escolher o número de nós para repassar as informações, em função do tamanho da vizinhança. Assim o Gossip2 se mostra bem flexível dependendo da quantidade de nós da rede e como a vizinhança nas simulações deste trabalho sempre apresenta variações, o Gossip2 é o mais eficaz [1].

- p1 é a probabilidade de um nó repassar o Gossip, se o número de vizinhos é maior que $n$. 
- k é o número de hops iniciais para os quais o Gossip é repassado com probabilidade 1 .

- p2 (p2>p1) é a probabilidade de um nó repassar o Gossip, se o número de vizinhos é menor que $n$.

- n é o ponto inicial (threshold) de número de vizinhos.

O algoritmo do Gossip2(p1, $k, p \mathscr{2}, n)[1]$ utilizado no protocolo AODV é mostrado abaixo:

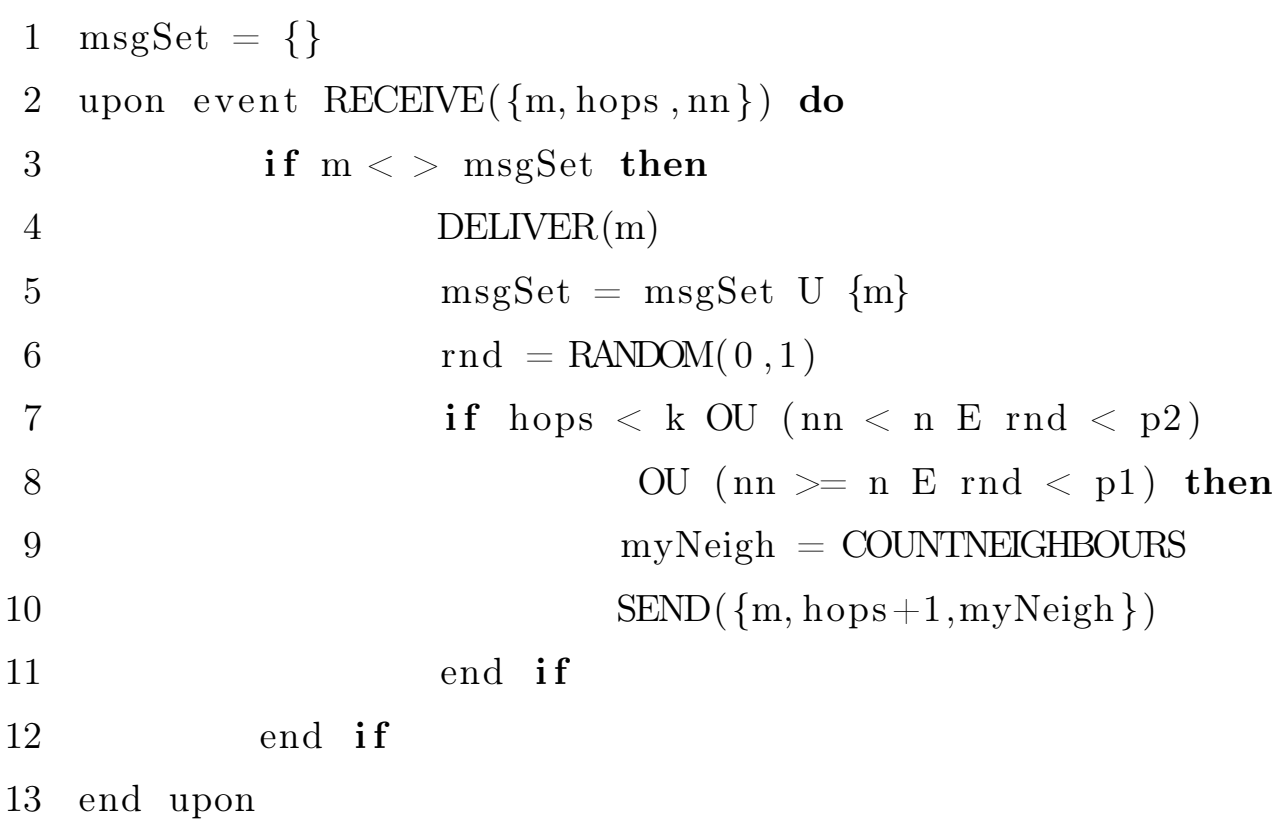

A implementação do Gossip2 com o protocolo DSR foi desenvolvida neste trabalho e tem como base o algoritmo acima.

Os valores das variáveis utilizados neste trabalho, para uma rede de tamanho médio de 80 nós e consequentemente um alcance médio de mensagens entre $50 \%$ e $80 \%$, foram:

- $\mathrm{p} 1=0,5$.

- $\mathrm{k}=3$.

- $\mathrm{p} 2=0,8$.

- $\mathrm{n}=4$.

Quando um nó recebe uma mensagem pela primeira vez, ele guarda a mensagem e a reenvia para um certo número de nós (fanout). Estes nós, por sua vez, 
selecionam alguns dos seus vizinhos e propagam a mensagem. Se um nó recebe uma mensagem que já conhece, ele a ignora.

A idéia básica do algoritmo Gossip é que todos participem da mesma maneira na disseminação da informação. Assim, se um nó deseja enviar uma mensagem broadcast, ele seleciona $f$ nós aleatoriamente e envia a mensagem a eles ( $f$ é o parâmetro fanout).
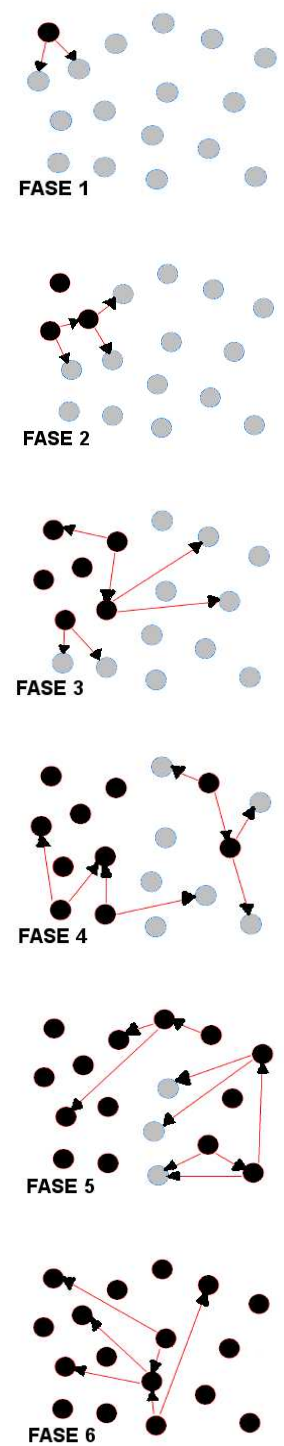

Figura 2.5: Passos da disseminação da informação no Gossip.

A Figura 2.5 ilustra como é feita a disseminação da informação pelos nós, passo a passo. Os nós pretos são os disseminadores e receptores das informações e os nós cinzas são os receptores. Os nós pretos selecionam um número médio de 2 vizinhos (inicialmente só os nós cinzas e posteriormente os nós cinzas e pretos). Esse número médio é determinado pela probabilidade de repasse de informação de 40-50\%. A informação enviada é representada pelos traços na figura. Os nós que recebem a informação, por sua vez, também selecionam mais 2 vizinhos para a disseminação da informação. O fato destes vizinhos já terem recebido a 
informação (nós pretos) não os impede de receber a mesma informação novamente. Isso é que faz do Gossip um algoritmo redundante. Por fim, todos os nós acabam recebendo a informação que são os nós pretos da fase 6 da Figura 2.3.

\subsection{Sistema de Quorum}

Um sistema de Quorum é uma coleção de subconjuntos de servidores proposta como uma ferramenta de replicação de dados ao longo dos anos.

Dado um conjunto de elementos $U$, um sistema de Quorum $Q$ é uma coleção de subconjuntos de $U$.

Um sistema de Quorum, basicamente, é constituído por ferramentas para se implementar alta disponibilidade de memória compartilhada distribuída. Possui como princípio que, se uma fonte de dados compartilhada é armazenada em um conjunto de servidores, então operações de leitura e escrita precisam ser implementadas somente em um subconjunto de seus servidores. A propriedade de intersecção de quorums assegura que cada leitor tenha acesso aos dados mais recentes. Qualquer prática de uso de sistema de Quorum deve contar com a possibilidade de que algum dos servidores possa falhar e que mesmo assim, eles devem trocar dados entre si e continuar funcionando.

Este trabalho apresenta um sistema composto de um número arbitrário de clientes e um conjunto $\mathrm{S}$ de servidores de dados. O número de servidores é fixo e em todos os cenários não são aplicadas falhas e nem adormecimento neste conjunto S de servidores.

Existe um sistema de Quorum Q, quando:

$$
Q=Q r+Q w
$$

Qr é um conjunto de quorums usado para operações de leitura (Quorum de leitura) e Qw é um conjunto de quorums usado para operações de escrita (Quorum de escrita). Qualquer par de quorums de leitura e escrita tem uma intersecção não nula.

Seu funcionamento se baseia em:

- para escrever dados em um servidor do Quorum, Figura 2.6, um cliente consulta os servidores do Quorum de leitura para escolher um timestamp 
que seja maior que os que já existam para qualquer dado, já salvo nos servidores, e envia os dados para os servidores do Quorum de escrita associado ao maior timestamp escolhido;

- para ler dados, um cliente faz a consulta nos servidores do Quorum de leitura, procura pelos dados mais recentes que são os que possuem maior timestamp e retorna os dados ao cliente. O sistema de Quorum utilizado neste trabalho é baseado no artigo [2].

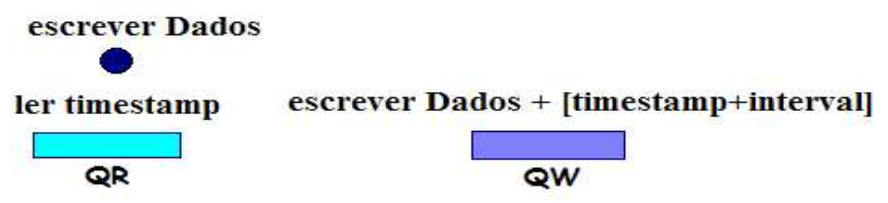

Figura 2.6: Sistema de leitura e escrita no Quorum

Em um sistema de Quorum, as operações de leitura são mais frequentes que as operações de escrita. Assim, o trabalho total do sistema de Quorum é medido pelo número total de acessos em disco ou pelo número total de mensagens.

Assumindo que um sistema de $n$ nós, na qual a escrita compreende uma fração $f_{w}$ de operações submetidas e a leitura compreende uma outra fração $f_{w}$. Então, o acesso médio de um sistema baseado em Quorum, com Quorum de leitura de $r$ nós e Quorum de escrita de $w$ nós, gera um trabalho total $(E)$ de [32]:

$$
E_{\text {Quorum }}=\left(1-f_{w}\right) \times r+f_{w} \times w
$$

\subsection{Parâmetros de Desempenho}

Os parâmetros de desempenho escolhidos, para avaliar um bom sistema de tolerância a falhas e medir as principais características que uma rede de dados deve possuir para o seu bom funcionamento, foram:

- RREQ: mensagens enviadas por um nó a um conjunto de nós da rede com o objetivo de encontrar uma rota. É medido o número total de RREQ transmitidos na rede.

- Vazão (bits/seg.): parâmetro que indica a taxa de transmissão efetiva dos dados em bits, medida em um determinado intervalo de tempo. 
- Latência (seg.): representa o tempo necessário para um pacote de dados ir de um dispositivo fonte até um dispositivo destino. É calculado pela soma dos tempos entre o envio e o recebimento dos fluxos Constant Bit Rate (CBR).

- Perda de pacotes (\%): representa a diferença entre o número de pacotes enviados e o número de pacotes recebidos, dado em valores percentuais.

- Consumo de energia (mWhr): dado pela soma da Energia consumida $\left(E_{y}\right)$ nos estados de Recepção de Dados (Rx), Transmissão de Dados (Tx), idle e sensing de informações.

O tempo gasto $(t)$ do sensor em certo estado depende do tamanho do pacote (PacketSize) e da taxa de transmissão (T $T_{x}$ Rate):

$$
t=\frac{\text { PacketSize }}{T_{x} \text { Rate }}
$$

Portanto, para cada estado, a energia consumida é calculada como:

$$
E_{y}=\alpha_{y} \times t_{y}
$$

$\alpha_{y}$ representa a potência dissipada pelo sensor no estado y, e $t_{y}$ representa o tempo gasto no estado y. O estado y pode ser: $\mathrm{Tx}, \mathrm{Rx}$, idle ou sensing. $\mathrm{O}$ valor de $\alpha_{y}$ é específico para cada sensor. 


\section{Desenvolvimento de Novo Algoritmo para Economia de Energia em Redes Ad-hoc}

Neste capítulo, serão descritos alguns algoritmos Power Aware existentes e o que é implementado neste trabalho, PWSave, juntamente com os seus testes de validação.

\subsection{Algoritmos Power Aware já existentes}

Devido à importância da economia de energia em dispositivos de rede, algoritmos que propiciem um menor consumo de bateria e que, ao mesmo tempo, não interfiram negativamente em parâmetros como latência, vazão e perda de pacotes são extremamente úteis e necessários nos dias atuais.

Abaixo serão abordados algoritmos Power Aware já implementados.

Em [6] é utilizada a energia restante dos nós como métrica de roteamento. A solução é desenvolvida na camada MAC por meio de um algoritmo que escolhe as rotas dependendo do maior nível de reservas energéticas dos nós. Foram feitas simulações com uma rede composta por 20 nós e foram apresentados resultados de uma redução máxima de custos (calculados após 300 segundos de simulação) de 5-45\%. Para cenários considerando baixa e alta carga na rede, o algoritmo não possui bons resultados de redução máxima de custos.

Em [8] são propostas duas novas versões do protocolo Dynamic Source Routing, mas há uma diminuição de 17\% em comparação com o DSR no cenário sem mobilidade; com o aumento da mobilidade dos nós da rede, o consumo de energia aumenta.

O DSR não se mostra um bom protocolo de roteamento para trabalhar com cenário móveis, como mostrado nesta dissertação. 
De acordo com [9] constata-se que a solução abrangendo um mecanismo de buffer duplo para economia de energia é válida, em que pacotes de broadcast e multicast podem ser entregues às estações com baixa carga da bateria. Há uma redução no consumo de energia de cerca de $50 \%$ em comparação com o mecanismo Power Saving implementado em [33]. Contudo, na avaliação da modalidade de baixa carga de bateria, apenas uma estação está com a bateria com baixa carga e no modo Power Saving; as outras estações estão em condições normais de carga de bateria.

Este trabalho distingue-se das soluções acima citadas por propor um algoritmo de economia de energia, PWSave, que não interfere no desempenho do conjunto de parâmetros (latência, vazão, perda de pacotes e RREQ), resultando em um menor consumo de energia em um cenário móvel, utilizando as características favoráveis do algoritmo Gossip no menor consumo de RREQ e do sistema de Quorum na diminuição de perdas de pacotes da rede.

\subsection{Algoritmo $P$ WSave}

Nesta dissertação foi implementado o algoritmo PWSave para trabalhar em conjunto com o protocolo de roteamento AODV no simulador Glomosim, em associação com o algoritmo Gossip e com o sistema de Quorum.

Para melhor compreensão do leitor, o algoritmo será representado por diagramas de estado (Figura 3.1, Figura 3.3, Figura 3.4 e Figura 3.5), os quais foram construídos utilizando a ferramenta freeware IDES 2.1 [34].

No diagrama de estados serão representados dois lados: o do servidor do Quorum e o do nó da rede. O nó da rede pode falhar ou não a qualquer momento e também pode entrar em estado de adormecimento ou permanecer acordado. O servidor do Quorum jamais adormece e tem o controle de todos os demais nós que não são servidores do Quorum. Portanto, se definindo o nó 8 como um servidor do Quorum, o algoritmo PWSave faz com que ele se comporte conforme os diagramas de estados das Figuras 3.1 e 3.4, caso contrário - ou seja, se o nó não for um servidor do Quorum - ele irá comportar-se conforme os diagramas de estados das Figuras 3.3 e 3.5 .

Existem duas fases no algoritmo PWSave: a associação do nó ao servidor do Quorum e o despertar ou adormecimento do nó se este, respectivamente, estiver ou não recebendo informações, sejam estas provenientes de dados ou de determinações de novas rotas. 


\subsubsection{Fase de Associação dos Nós ao Sistema Servidor do Quorum}

O funcionamento da fase de associação dos nós ao sistema servidor do Quorum é mostrado nas Figuras 3.1 e 3.3.

A Figura 3.1 mostra o diagrama de estados do algoritmo PWSave, referente à fase de associação, no lado do servidor do Quorum.

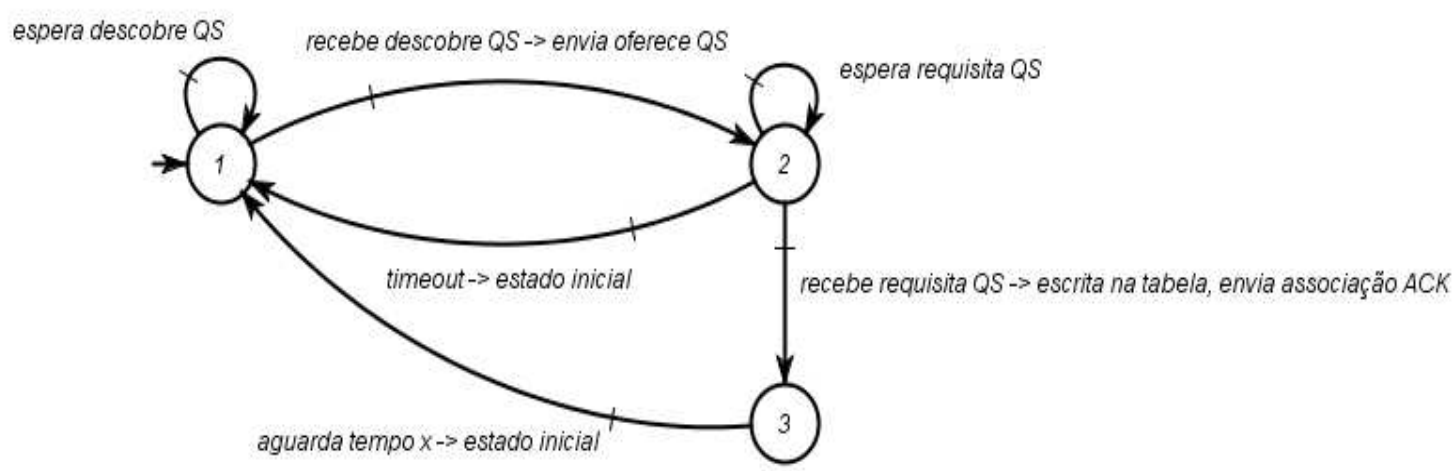

Figura 3.1: Diagrama de estados da fase de associação do algoritmo PWSave implementado no lado do servidor do Quorum.

No estado 1, o servidor do Quorum aguarda pelos pedidos de associação dos nós (evento espera descobre Qs). Neste momento, nenhum dos nós está associado e cada um deles precisa descobrir a qual servidor do Quorum deverá associarse. Após o recebimento do pedido de associação, mensagem descobre Qs enviada pelo nó, o servidor do Quorum envia uma mensagem de resposta ao nó chamada oferece Qs que indica que o nó pode se associar a ele. A seguir, o servidor do Quorum faz uma transição para o estado 2 onde fica aguardando a mensagem requisita $Q s$, que indica que o nó ainda não está associado e aceita associar-se a este servidor do Quorum. Recebendo a aceitação da associação do nó (mensagem requisita $Q s$ ), o servidor do Quorum escreve em uma tabela que esse determinado nó está associado a ele; essa tabela é útil na hora da notificação dos seus nós associados, para informar se estes podem adormecer ou não; também é enviada uma mensagem Acknowledgement (ACK) do servidor do Quorum para o nó, para informar que ele já está associado e não deve pedir uma nova associação. Ao enviar estas mensagens, chega-se ao estado 3, que após transcorrido um tempo de 51 segundos, retorna ao estado 1, estado de espera dos novos pedidos de associação dos nós.

As novas associações devem ser refeitas, dado que a cada certo tempo os nós se movimentam tornando necessária a associação a um novo servidor do 
Quorum. Este tempo foi escolhido conforme o cenário de mobilidade não muito alta, ocorrendo de 51 em 51 segundos.

No caso do timeout, tempo de esgotamento para que o estado 2 retorne ao estado 1, considera-se como sendo de $135 \mathrm{~ms}$, Figura 3.2, que é a soma dos tempos de chegada das mensagens, levando em consideração que o tempo máximo de chegada de cada mensagem até o nó seja de 40 ms; foi considerado um possível timeout. Portanto, o timeout pode ocorrer devido à mensagem requisita Qs ter se perdido ou se é momento de se fazer novas associações.

nó 0 se associa a QS1
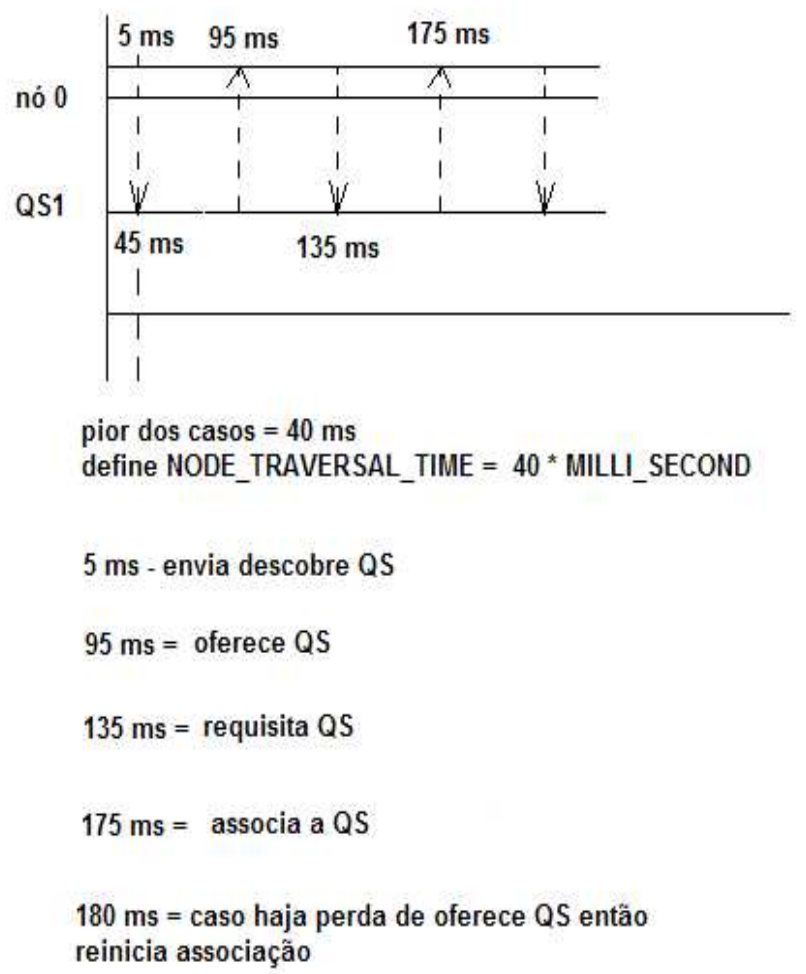

Figura 3.2: Diagrama de tempo de associação do nó com o servidor do Quorum.

Mais de um servidor do Quorum pode receber o pedido de associação de um mesmo nó, porém a associação ocorrerá de acordo com a menor distância entre o nó e o servidor do Quorum. Assim que um nó estiver associado, passará a descartar as respostas de associação dos outros servidores do Quorum.

Na Figura 3.3 é mostrado o diagrama de estados do algoritmo PWSave referente à fase de associação no lado do nó que não pertence ao conjunto dos servidores do Quorum. No estado 1, aguarda-se um determinado tempo (5 ms) para ir ao estado 2, tempo este que é necessário para a verificação das coorde- 
nadas x, y e z de cada nó, definidas aleatoriamente. O nó sai do estado 1 e envia uma mensagem descobre Qs aos nós servidores do Quorum, para descobrir com qual deverá associar-se. No estado 2, aguarda pela resposta/mensagem oferece $Q s$ do servidor do Quorum, que após ter recebido esta mensagem envia requisita Qs ao servidor do Quorum mudando para o estado 3. O nó permanecerá no estado 3 até receber uma mensagem ACK do servidor do Quorum, mensagem esta que significa que o nó acaba de associar-se ao servidor do Quorum mais próximo, indo para o estado 4. Caso receba outras associações seguidas, estas serão ignoradas.

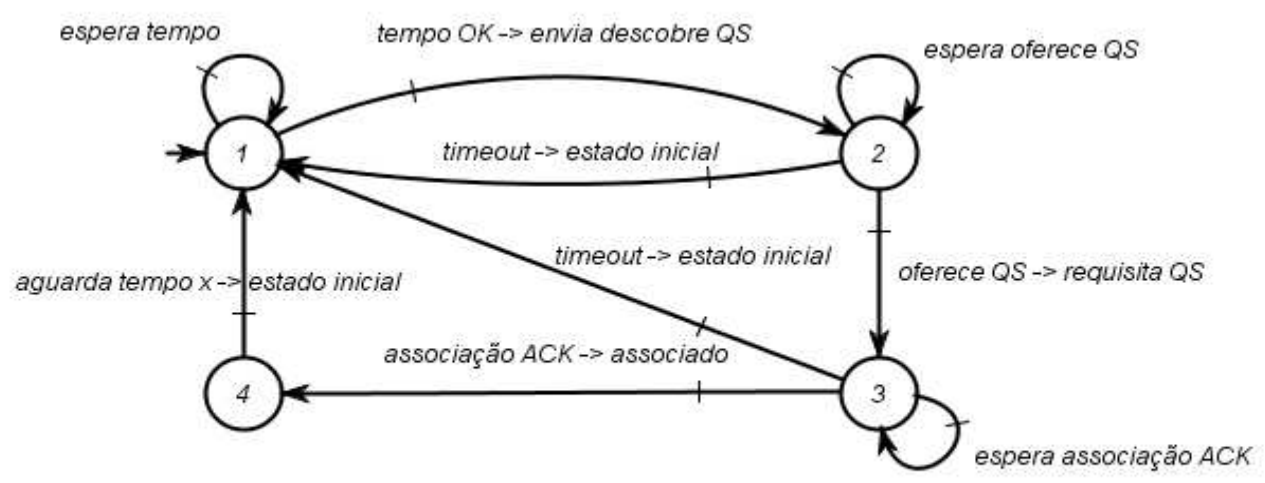

Figura 3.3: Diagrama de estados da fase de associação do algoritmo PWSave implementado no lado do nó não pertencente ao conjunto do servidor do Quorum.

Após, um determinado tempo (51 segundos)a máquina retorna para o estado 1, em que é feito um novo pedido de associação.

Os timeout para que os estados 2 e 3 retornem ao estado 1 são, respectivamente, de 95 e 180 ms, definidos na Figura 3.2.

Após a Fase de Associação, a máquina segue para a Fase de Adormecimentos dos nós.

\subsubsection{Fase de Adormecimento dos Nós que não pertencem ao conjunto do servidor do Quorum}

O funcionamento desta fase é mostrado nas Figuras 3.4 e 3.5.

A Figura 3.4 mostra o diagrama de estados do algoritmo PWSave referente à fase de adormecimento dos nós, no lado do servidor do Quorum. O estado 1 é quando o servidor do Quorum aguarda pelos pedidos de RREQ ou RREP, que também são enviados ao Quorum, indicando que um determinado nó vai receber 
informações ou fará parte da determinação de uma nova rota e, portanto, o nó não deve adormecer. Ao receber as mensagens RREQ ou RREP a máquina passa ao estado 2, atualizando a tabela de nós ativos, significando que os nós envolvidos com as mensagens RREQ ou RREP devem ficar acordados. Após a atualização da tabela, os nós que devem permanecer acordados são notificados pelo servidor do Quorum, passando para o estado 3 (nó deve permanecer acordado), que após um determinado timeout, $105 \mathrm{~ms}$, retorna ao estado 1 . O timeout do estado 1 para o estado 3 é de $95 \mathrm{~ms}$. Estes valores, 105 e $95 \mathrm{~ms}$, foram escolhidos pelos testes de simulação, conforme Figuras 4.3 e 4.4, considerando um tempo máximo para que um nó permaneça acordado ou adormecido, respectivamente, sem prejudicar parâmetros como latência, perda de pacotes e vazão.

Caso um determinado nó decida receber ou enviar dados na rede, este nó deve aguardar $500 \mathrm{~ms}$ até que esta requisição chegue ao fim. Este tempo foi escolhido segundo o tamanho do pacote enviado na rede definido como sendo de 546 Bytes (512 de dados +34 de header), utilizando uma taxa de $2 \mathrm{MB} / \mathrm{s}$. Assim, como um pacote de 546 Bytes deve chegar ao seu destino em um tempo de $273 \mathrm{~ms}$, o tempo escolhido de $500 \mathrm{~ms}$ é suficiente para que o pacote enviado seja entregue ao seu nó destino.

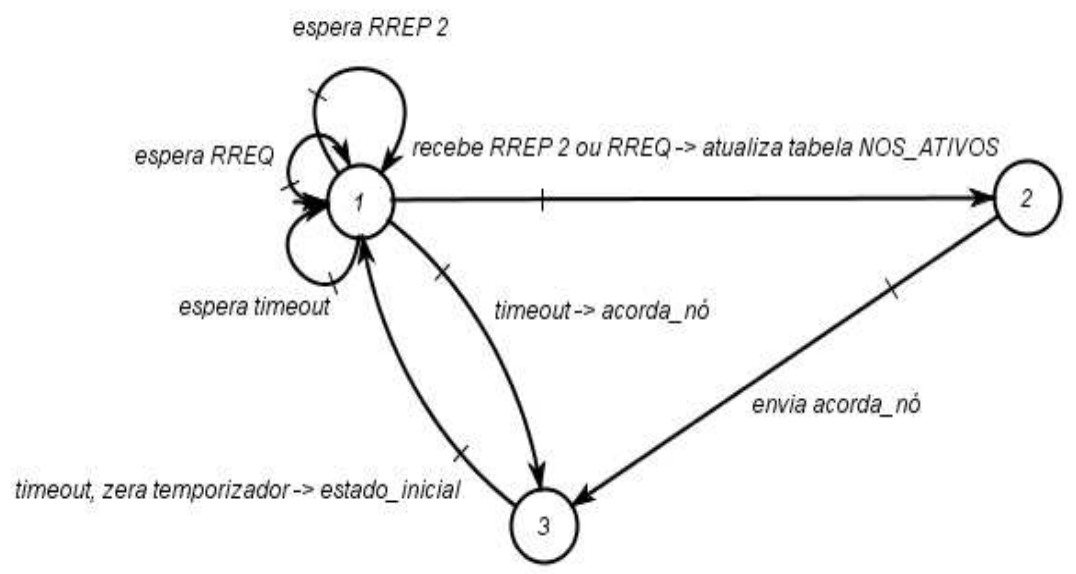

Figura 3.4: Diagrama de estados da fase de adormecimento do algoritmo PWSave implementado no lado do servidor do Quorum.

Na Figura 3.5, mostra-se o diagrama de estados do algoritmo PWSave referente à fase de adormecimento no lado do nó que não pertence ao conjunto dos servidores do Quorum. O estado 1 representa o nó da rede acordado, aguardando por requisições de RREQ, RREP e por dados. Caso estes não cheguem, após um determinado timeout, $105 \mathrm{~ms}$, o nó pode ir para o estado 3 de adormecimento, mas se o estado 1 recebe alguma requisição, o nó deve aguardar 500 ms até que 
esta requisição chegue ao fim, dando tempo para que a informação se dissemine ou para a recepção dos dados. No estado 3, caso o servidor do Quorum ao qual o nó está associado receba informação de que este determinado nó fará parte de alguma próxima requisição, o servidor do Quorum envia para o nó a informação de que ele deve se manter acordado, retornando ao estado 1 . No estado 2, depois que o nó recebe uma RREQ deverá gerar, duplicar (RREP1) e enviar para o servidor do Quorum um RREP2, para que este saiba se os nós associados a ele farão parte de novas rotas e/ou receberão dados, caso em que este(s) nó(s) deverá(ão) permanecer acordado(s). No estado 4, caso a RREQ seja perdida, um RREQ, após 330 ms, será gerada. Neste estado, a duplicação da RREQ já vêm implementada pelo protocolo padrão do AODV.

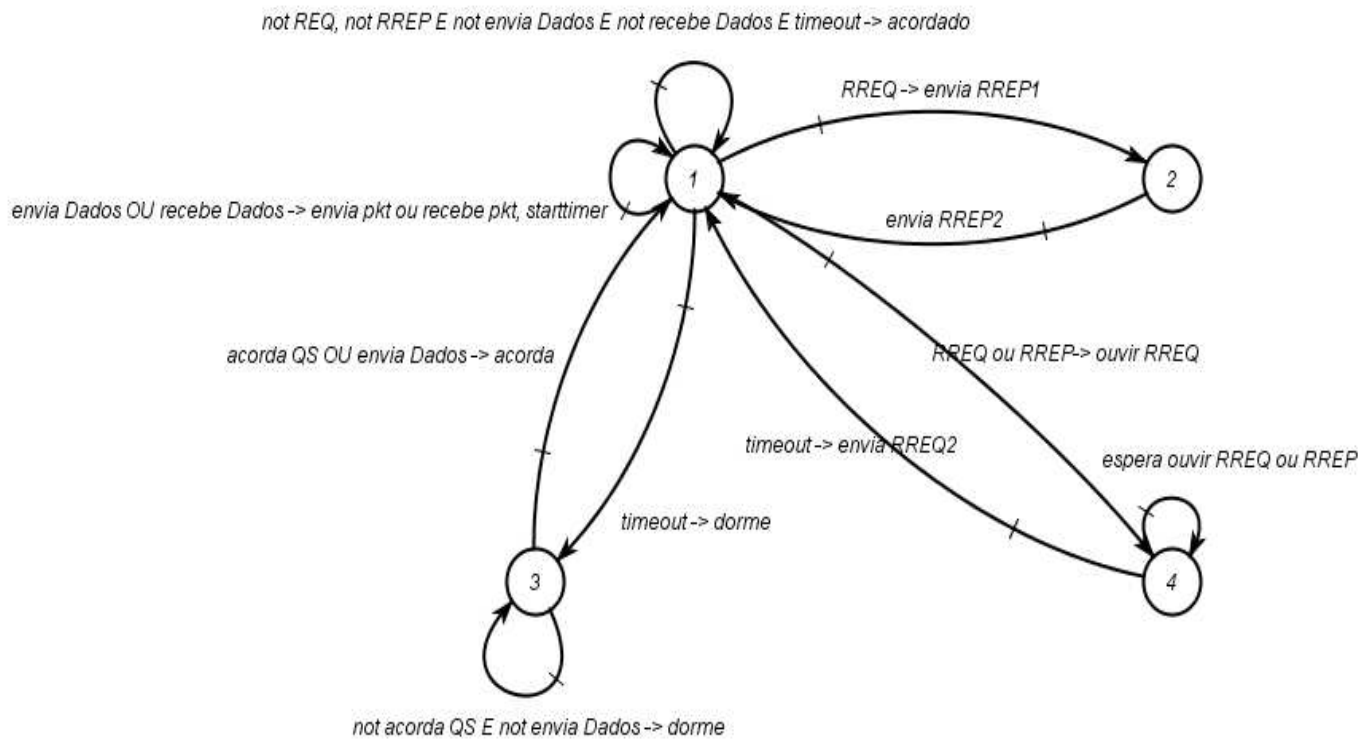

Figura 3.5: Diagrama de estados da fase de adormecimento do algoritmo $P W S a v e$ implementado no lado do nó não pertencente ao conjunto do servidor do Quorum.

\subsubsection{Validação do Algoritmo PWSave}

Para validação do algoritmo PWSave foram feitos testes com um número inicial de 6 nós, sendo que dois destes nós (nó 0 e nó 5) são definidos como servidores do Quorum. No cenário de teste, o nó 1 envia um tráfego CBR ao nó 3, sendo que o nó 1 está associado ao nó 0 (servidor do Quorum) e o nó 3 está associado ao nó 5 (servidor do Quorum). Este cenário é representado pela Figura 3.6 .

Inicialmente, tem-se um cenário com mobilidade pré-definida que ocorre após 


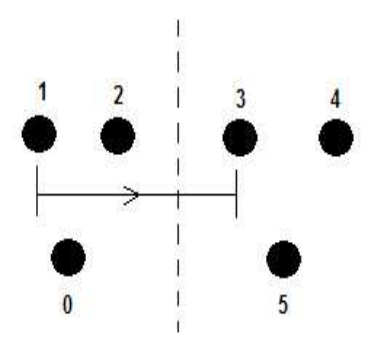

Figura 3.6: Cenário inicial de testes de 6 nós

0, 50 e 200 segundos. A associação deve, portanto, ocorrer após esses valores.

Posteriormente, neste trabalho, foi modificado o tempo de reassociação para que ocorresse a cada 51 segundos.

O gráfico da Figura 3.7 valida o momento de associação do nó 1 ao nó 0 , servidor do Quorum, que ocorre um pouco depois de 0, 50 e 200 segundos. O mesmo ocorre com o nó 3, que se associa com o nó 5, servidor do Quorum.

A reassociação é necessária, uma vez que se trata de um cenário de mobilidade e os nós podem deslocar-se e sempre devem associar-se aos Quorums mais próximos.

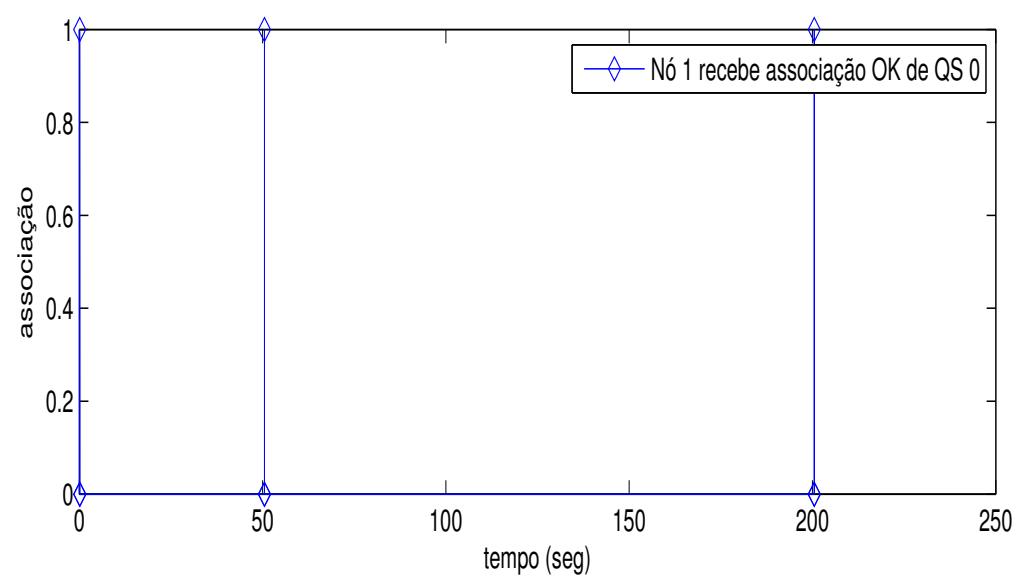

Figura 3.7: Momento de associação do nó 1 ao nó 0

O gráfico da Figura 3.8 mostra os testes de associação dos nós com os servidores do Quorum, nó 0 definido como QS0 ( Quorum 0) e nó 5 como QS5 (Quorum 5), utilizando a mobilidade que é representada pelas setas, indicando a posição anterior e posterior do nó ao ocorrer a mobilidade. Os nós 1 e nó 2 estão mais próximos a QS0 e, portanto, associam-se a este servidor do Quorum. Quando se executa o simulador, uma lista que mostra a associação é impressa e é verificada a associação dos nós 1 e 2 ao QS0. O mesmo ocorre com os nós 3 e 4, que por estarem mais próximos de QS5 associam-se a ele. 


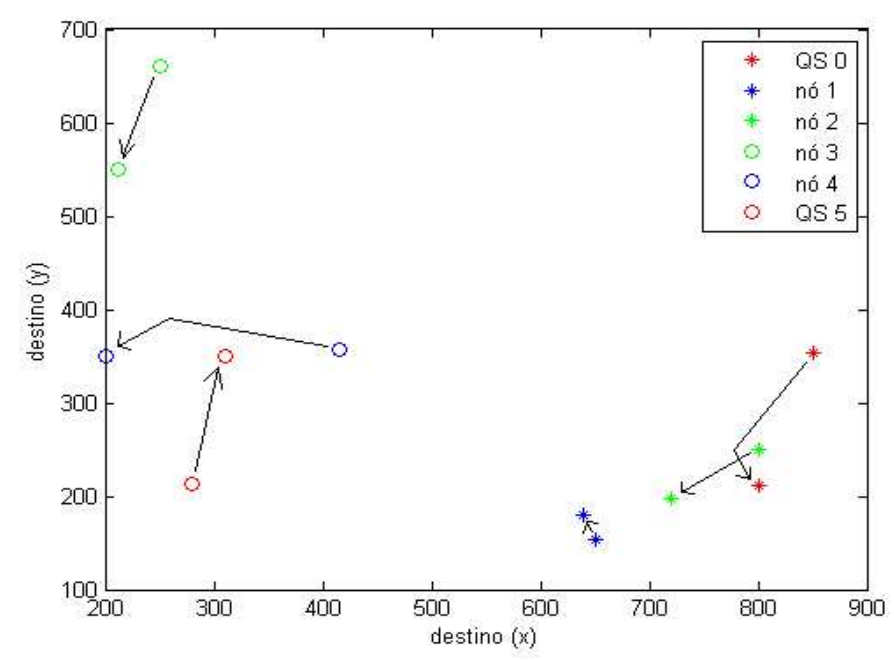

Figura 3.8: Mobilidade dos nós

A Figura 3.9 mostra os pacotes CBR enviados do nó 1 para o nó 3. Quando a coordenada y é igual a 0 significa que não há tráfego de dados na rede, porém se há tráfego na rede a coordenada y é igual a 1. Como o tráfego de dados, neste cenário, foi enviado de 10 em 10 segundos, com início a partir de 11 segundos, o nó 3 recebe os dados após 11 e 21 segundos. Na Figura 3.9 comprova-se que apesar dos nós entrarem em estado de adormecimento, os pacotes são recebidos pelo nó 3, o que indica que os nós estão acordados nos momentos de envio e recepção dos pacotes.

O tempo de simulação mostrado nas Figuras 3.9, 3.10, 3.11 e 3.12 vai até 22 segundos, uma vez que após este tempo o processo de dorme/acorda e recepção de pacotes se repete com um comportamento similar ao tempo anterior aos 22 segundos.

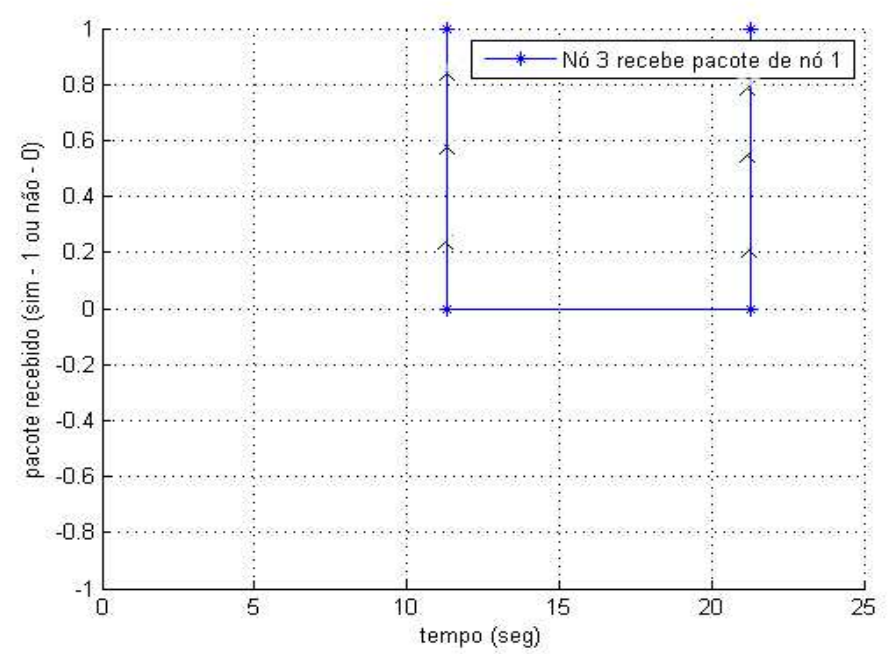

Figura 3.9: Nó 3 recebendo pacotes no nó 1 


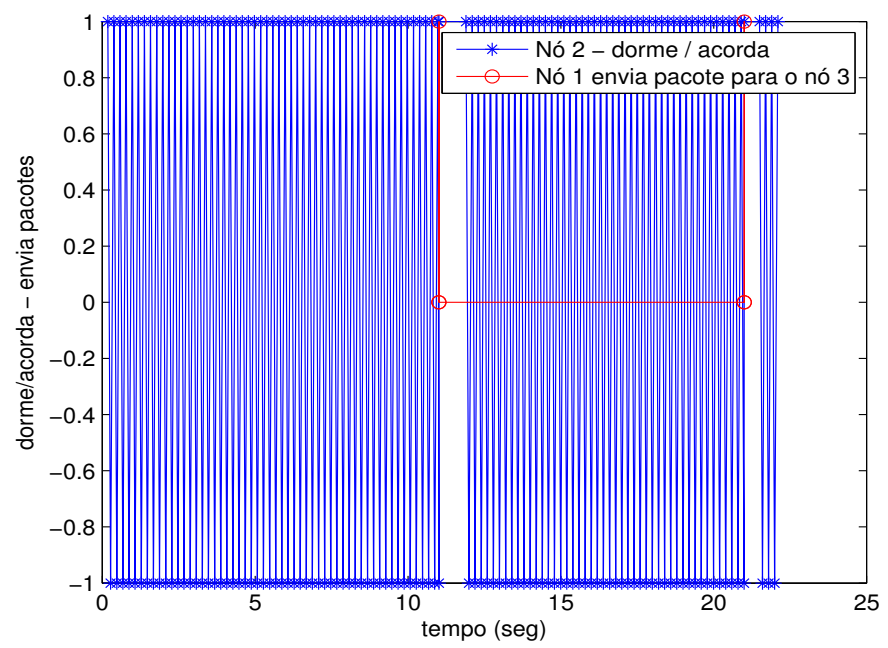

Figura 3.10: Estado de dorme/acorda do nó 2 e envio de pacotes do nó 1 para o nó 3

Na Figura 3.10 é mostrado o estado de dorme e acorda do nó 2, como ele está entre o nó 1 e o nó 3 no momento em que o pacote é enviado ao nó 3, o nó 2 deve estar no estado acordado, dado que ele faz parte da rota para se chegar até o nó 3. As linhas azuis da Figura 3.10 indicam que o nó 2 dorme $(\mathrm{y}=-1)$ durante $95 \mathrm{~ms}$ e mantém-se acordado ( $\mathrm{y}=1$ ) durante $105 \mathrm{~ms}$, periodicamente, caso nenhuma mensagem de RREQ, RREP ou nenhum dado seja enviado. No momento em que o nó 1 envia o pacote $(\mathrm{y}=1)$ para o nó 3 , o nó 2 está no estado acordado e permanece até um determinado tempo, $500 \mathrm{~ms}$, para garantir que a mensagem tenha sido realmente enviada.

A Figura 3.11 mostra que durante a recepção dos dados pelo nó 3, este mantém-se acordado, garantindo a entrega de pacotes.

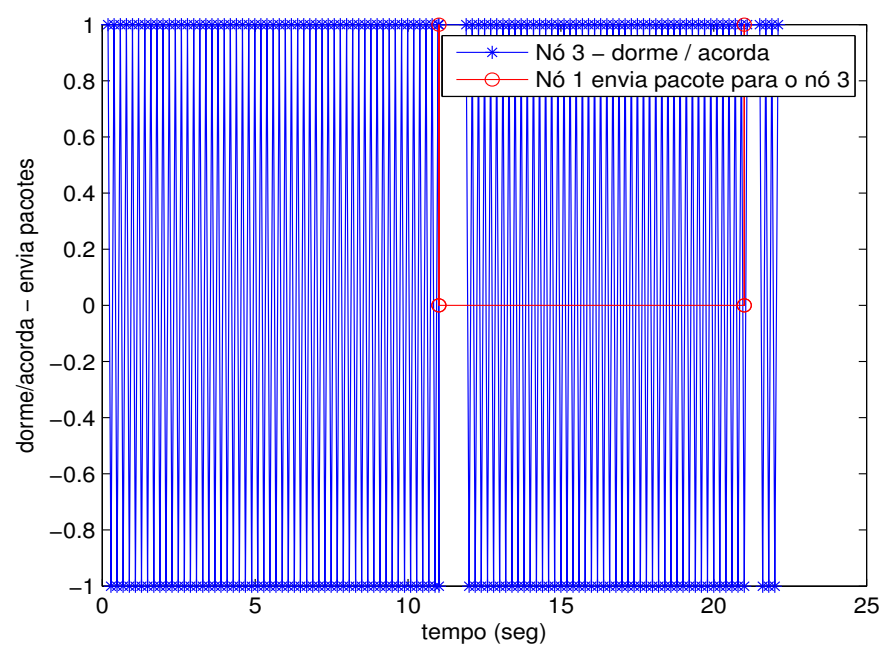

Figura 3.11: Estado de dorme/acorda do nó 3 e envio de pacotes do nó 1 para o nó 3 
A Figura 3.12 mostra que o nó 4 não se mantém acordado durante o envio de pacotes para o nó 3 porque o nó 4 não faz parte da rota escolhida para chegar até o nó 3. Portanto, ele pode continuar em seu estado de dorme/acorda mesmo durante o envio de pacotes na rede.

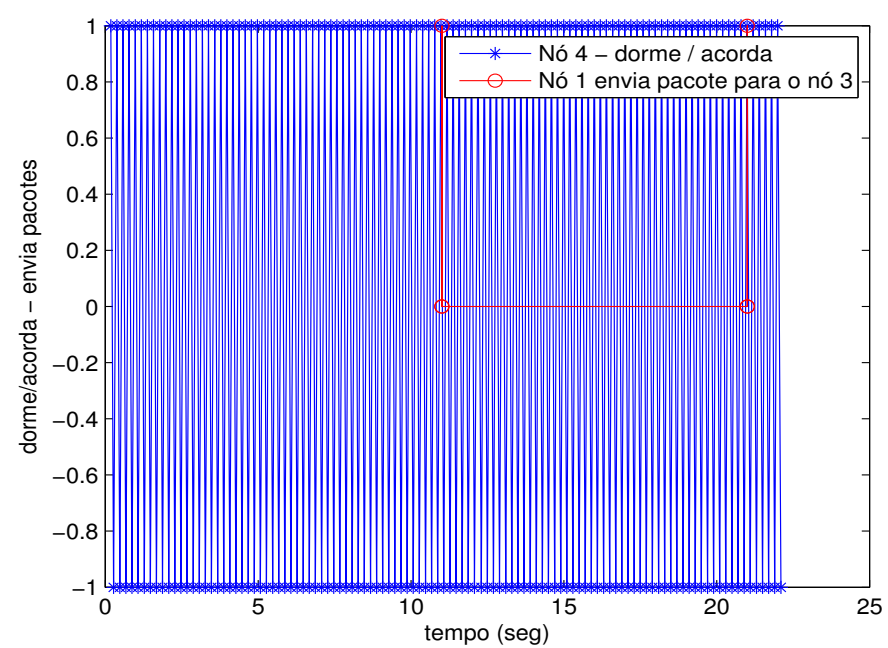

Figura 3.12: Estado de dorme/acorda do nó 4 e envio de pacotes do nó 1 ao nó 3

Após a validação do algoritmo PWSave com o cenário teste de 6 nós, fez-se a simulação com o cenário final de 80 nós e os resultados obtidos seguem o mesmo comportamento do cenário de 6 nós. Portanto, o algoritmo $P$ WSave é válido, de acordo com os testes feitos de associação ao servidor do Quorum, adormecimento dos nós, envio e recepção de pacotes. 


\section{Resultados Experimentais}

Este capítulo descreve os cenários de simulação abordados neste trabalho com o algoritmo Gossip, Sistema de Quorum e o algoritmo PWSave, juntamente com os testes realizados com os parâmetros RREQ, perda de pacote, vazão, latência e consumo de energia.

\subsection{Simulador}

Foram analisados dois simuladores: NS-2 [35] e Glomosim 2.03 [36] versão educacional. Ambos são softwares livres e têm sido amplamente utilizados em pesquisas, servindo como referências para comparações.

Testes com o NS-2 mostraram que ele é mais lento ao executar as simulações e gera arquivos com os resultados das simulações bem maiores quando comparados ao Glomosim, enquanto o NS-2 gera um arquivo de 4 MB, o Glomosim gera um arquivo de $12 \mathrm{~KB}$. Como várias simulações foram efetuadas neste trabalho, optouse pelo simulador que gerasse arquivos menores e que efetuasse as simulações com maior rapidez.

A plataforma de simulação Glomosim é uma biblioteca que quando compilada e utilizada permite fazer simulações de forma muito escalável - ou seja, com muitos nós de rede - para arquiteturas de redes fixas e, principalmente, para redes móveis wireless. Assim, a biblioteca GlobalMobile Information System Simulation Library (Glomosim) foi projetada aproveitando a capacidade de simulação de eventos paralelos, fornecida pelo PARSEC, da Universidade da UCLA, permitindo o processamento paralelo. Com isso, para executar a biblioteca de funções do Glomosim é necessário um compilador C como o GNU C Compiler (GCC), e uma versão do compilador PARSEC ou PCC, que atualmente já vem incluído no pacote de distribuição do Glomosim.

O Glomosim, pelas características já citadas acima e por ser utilizado em [1] e [37], que servem como importantes referências para este trabalho, foi o simulador 
escolhido para a construção do cenário de simulação.

\subsection{Cenário de Simulação}

A seguir serão abordados todos os cenários de simulação implementados no simulador Glomosim. Todos os cenários trabalham com uma rede de tamanho médio de 80 nós e um tráfego de 30 fluxos CBR entre os nós determinados para fins de comparação com o trabalho [1].

\subsubsection{Cenário de Implementação do algoritmo Gossip e Sis- tema de Quorum, sem falhas}

O cenário de simulação foi definido como:

- área de 1000 m x 1000 m;

- tempo de simulação: 2, 5 e 10 minutos;

- número de nós: 80, uniformemente distribuídos em uma grade de grau = 11,7 (número médio de vizinhos imediatos);

- tráfego: conjunto de 30 fluxos CBR entre pares aleatoriamente selecionados; a uma taxa de 2 pacotes/segundo; cada pacote com 512 bytes;

- mobilidade: cinco tipos de mobilidade foram selecionados segundo o tempo de pausa: 50, 100, 150, 200 e 250 segundos e um cenário sem mobilidade (considerado como 300 segundos).

- protocolos: as combinações dos seguintes protocolos e algoritmos foram simuladas:

$$
\begin{aligned}
& - \text { AODV; } \\
& - \text { DSR; } \\
& - \text { AODV + Gossip; } \\
& - \text { DSR + Gossip; } \\
& - \text { AODV + Gossip + Quorum; } \\
& \text { - DSR + Gossip + Quorum. }
\end{aligned}
$$

Os tempos de simulação de 2, 5 e 10 minutos foram testados e observou-se que o tempo de 5 minutos foi suficiente para que a rede alcançasse um regime de 
estado estacionário em todos os cenários definidos. Portanto, utilizou-se o tempo de simulação de 5 minutos nas demais simulações.

Para cada situação de interesse, 100 simulações para o protocolo AODV e 150 simulações para o protocolo DSR foram feitas com diferentes valores de semente (seed) para cada gerador de número aleatório. Em todos os casos, foram avaliados o desempenho da média e do desvio padrão. O histograma, Figura 4.1, que mostra as simulações para o parâmetro RREQ do protocolo DSR, com e sem Gossip, com um tempo de pausa entre os deslocamentos de 50 segundos; a função qq-plot de cada simulação foi feita para verificar se os resultados seguiam uma distribuição Normal. A normalidade dos resultados foi constatada.

Todos os resultados foram tratados pelo software estatístico $R$ [16].
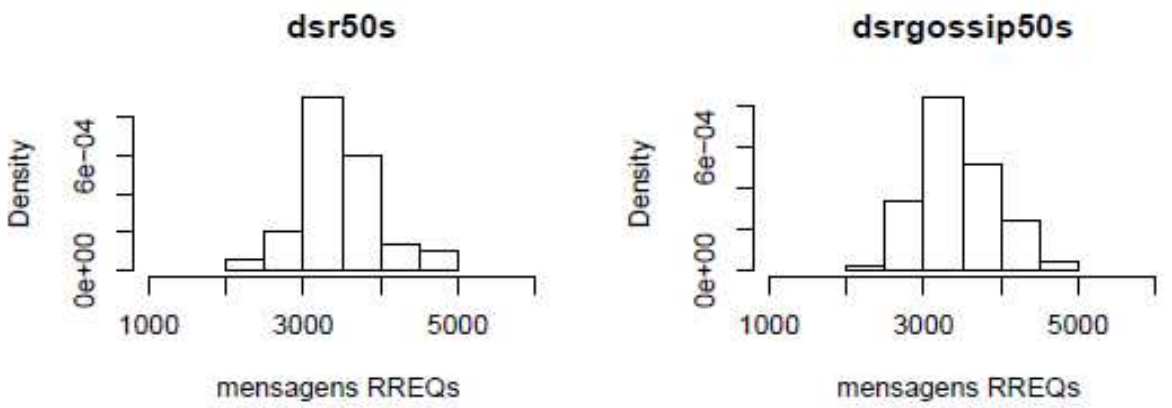

Figura 4.1: Histograma das simulações para o parâmetro RREQ do DSR, com e sem Gossip, com um tempo de pausa entre os deslocamentos de 50 segundos.

O protocolo de roteamento DSR foi utilizado somente nas soluções com Gossip e sistema de Quorum sem falhas. Os demais cenários com falhas e PWSave foram simulados somente com o protocolo de roteamento AODV, pois o DSR apresentou resultados insatisfatórios, como será abordado em detalhe posteriormente, na seção dos resultados.

\subsubsection{Cenário de Implementação de Falhas}

A simulação considerou os seguintes parâmetros:

- área de 1000 m x 1000 m;

- tempo de simulação: 5 minutos;

- número de nós: 80, uniformemente distribuídos ao longo de uma grade de grau $=11,7$ (número médio de vizinhos imediatos); 
- tráfego: conjunto de 30 fluxos CBR entre pares aleatoriamente selecionados. A uma taxa de 2 pacotes/segundo; cada pacote com 512 bytes;

- mobilidade: cinco tipos de mobilidade foram selecionados segundo o tempo de pausa: 50, 100, 150, 200 e 250 segundos e um cenário sem mobilidade (considerado como 300 segundos).

- protocolos: as combinações dos seguintes protocolos e algoritmos foram simuladas:

$$
\begin{aligned}
& -\mathrm{AODV} \\
& -\mathrm{AODV}+\text { Gossip } \\
& -\mathrm{AODV}+\text { Gossip }+ \text { Quorum. }
\end{aligned}
$$

Dois cenários de simulação de falhas, designados como A e B, são definidos e descritos a seguir.

- Cenário A:

- Depois de 2,30 minutos de simulação, um grupo de nós é forçado a falhar por um tempo constante de 10 segundos. A falha é simulada apagando os dados do nó e deixando-o indisponível por 10 segundos.

- Probabilidade de falha: as falhas aplicadas são de 7,5, 15, 22,5 e $30 \%$ sobre os nós pré-definidos. Por razões de simplicidade, a origem e destino CBR dos nós nunca falham.

A Tabela 4.1 apresenta os nós pré-definidos, que são forçados a falhar no cenário A.

- Cenário B:

- A simulação inicia e depois de 50 segundos um certo número de nós falham. A duração F1 da falha é aleatoriamente selecionada de uma distribuição exponencial, calculada pelo software R. Depois de F1, outro grupo de nós falham por F2 segundos. O tempo de simulação nunca excede os 5 minutos.

- Probabilidade de falhas: os nós são aleatoriamente selecionados de uma distribuição uniforme com uma média de probabilidade de 7,5 , 15, 22,5 e 30\%. Por razões de simplicidade, a origem e destino CBR dos nós nunca falham. 
Tabela 4.1: Falhas do Cenário A

\begin{tabular}{cc}
\hline Falhas (\%) & $\begin{array}{c}\text { Nós fixados com } \\
\text { ID de falhas }\end{array}$ \\
\hline 7,5 & $1,14,22,47,63,79$ \\
15 & $1,7,14,17,22,31$, \\
& $39,47,54,63,74,79$ \\
22,5 & $1,4,7,11,14,17,22$, \\
& $26,31,34,39,44,47$, \\
& $54,59,63,74,79$ \\
30 & $1,4,7,9,11,14,17,19$, \\
& $22,26,29,31,34,39,41$, \\
& $44,47,51,54,59,63,69,74,79$
\end{tabular}

\subsubsection{Cenário de Implementação do algoritmo PWSave}

O cenário de simulação final do PWSave é semelhante ao cenário de implementação do Gossip e Sistema de Quorum, com exceção da taxa dos dados injetados periodicamente na rede, que passa de 2 pacotes/segundo para 1 pacote/segundo, pois os testes referentes à escolha do tempo de adormecimento e despertar foram efetuados a 1 pacote/segundo.

Os servidores do Quorum são dispostos de maneira fixa na área de $1000 \mathrm{~m} \mathrm{x}$ $1000 \mathrm{~m}$ conforme a Figura 4.2 .

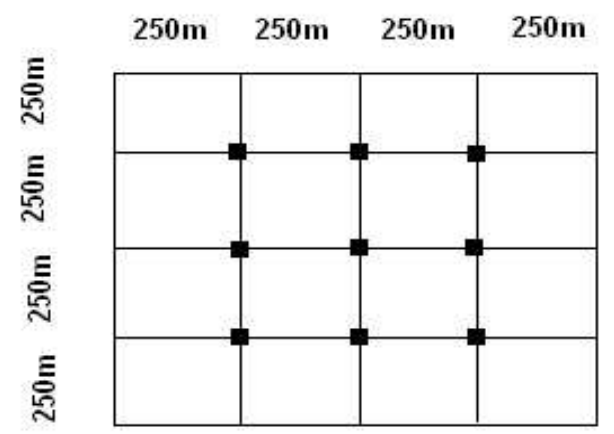

Figura 4.2: Posição dos nós servidores do Quorum

Os servidores do Quorum nunca falham e o tempo de adormecimento dos nós utilizado nas simulações foi de $90 \mathrm{~ms}$. Antes de escolher este tempo foram feitos testes com os tempos de adormecimento: 0, 30, 60, 70, 80, 85, 90, 95, 100, 110, 120, 130 e 140 ms nos diferentes tempos de mobilidade no cenário sem falha. 


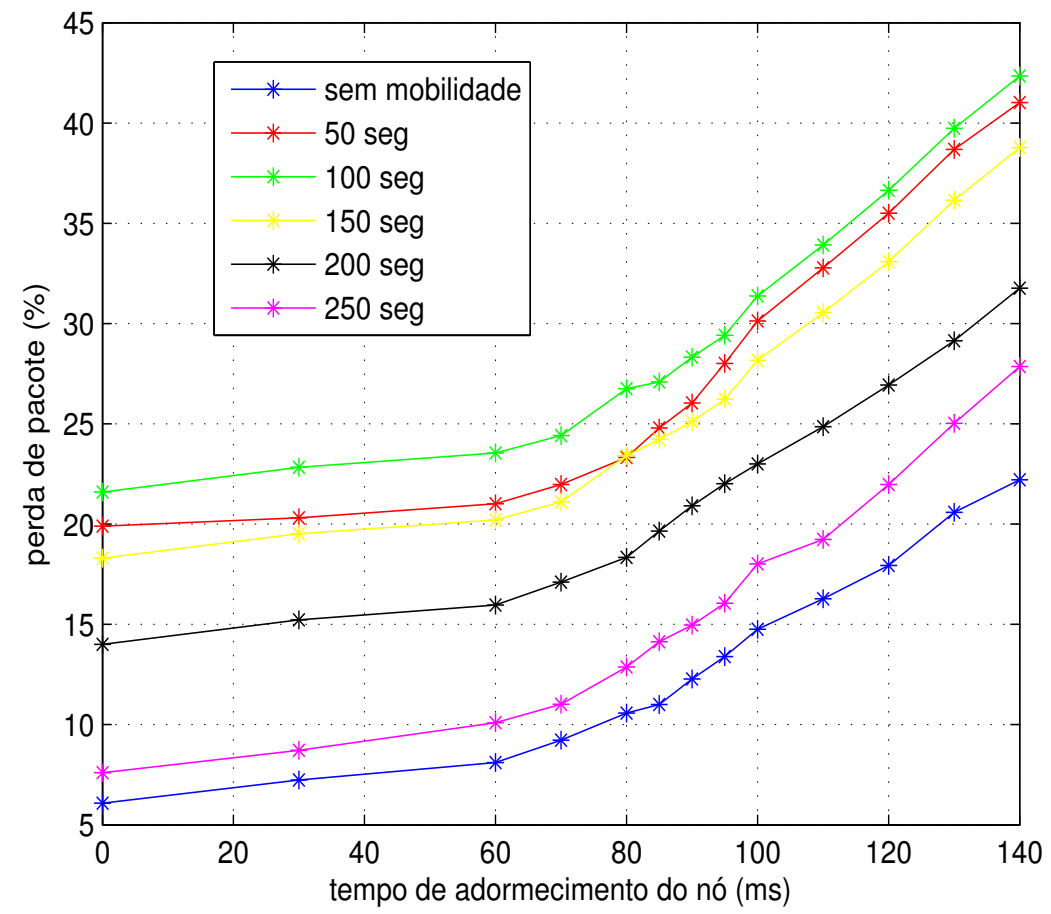

Figura 4.3: Perda de pacotes nos diferentes tempos de adormecimento dos nós

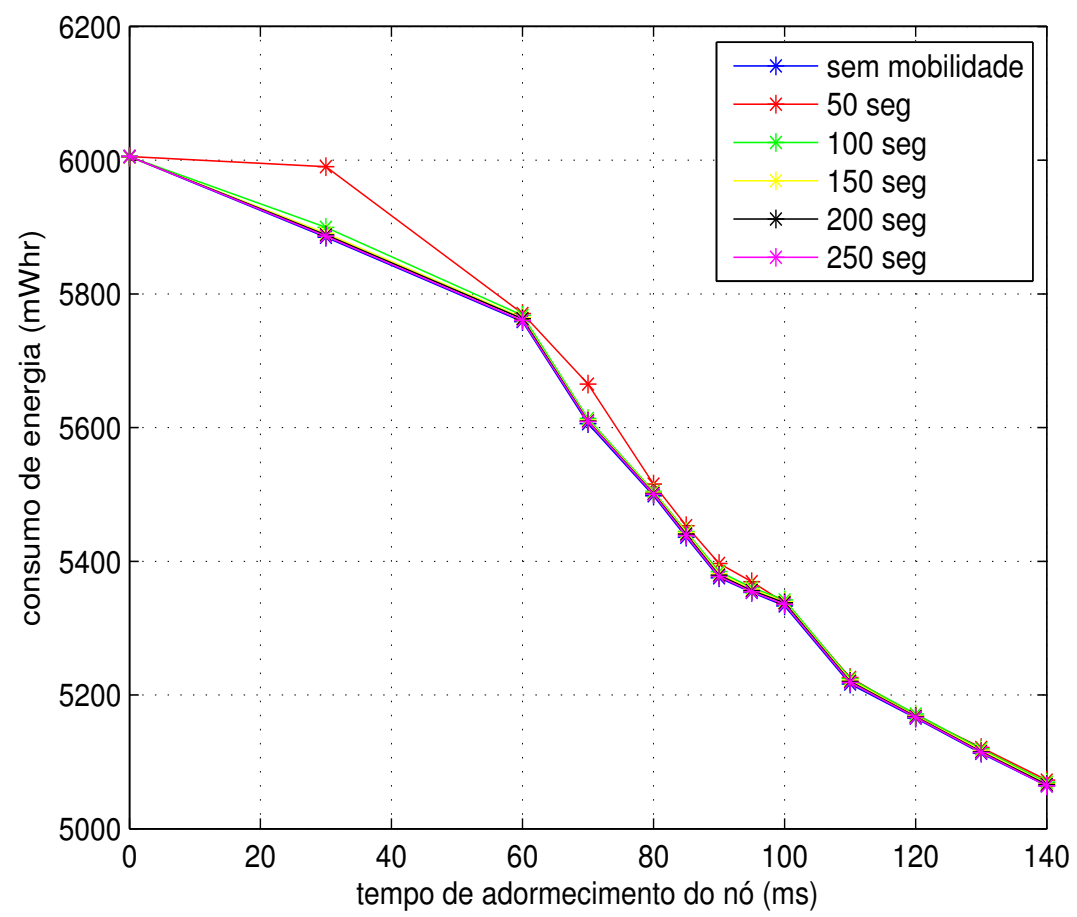

Figura 4.4: Consumo de energia nos diferentes tempos de adormecimento dos nós

As Figuras 4.3 e 4.4 mostram uma economia de energia em torno dos $10 \%$ com uma perda de pacotes em torno dos 5\% em um tempo de 90 ms sem mobilidade, portanto, foi escolhido o tempo de adormecimento total dos nós de 90 ms, por ser o melhor resultado do maior número de economia de energia em relação à menor 
perda de pacotes.

Primeiramente, foi feita a simulação sem aplicação de falhas e, posteriormente, as falhas foram introduzidas segundo o cenário já explicado anteriormente, na seção do Cenário de Implementação de Falhas.

A equação 4.1 define um índice de mérito cujo objetivo é o de avaliar o desempenho do algoritmo PWSave em termos de uma média ponderada entre o consumo de energia e um dos parâmetros monitorados nesta dissertação, isto é, latência, vazão, perda de pacotes e RREQ. O parâmetro de ponderação é expresso pela variável $\alpha$.

$$
\text { Indice }_{x}=\alpha \times \frac{\text { ParamRef }}{\text { Param }}+(1-\alpha) \times \frac{\text { ConsumoEnergiaRef }}{\text { ConsumoEnergia }}
$$

Onde:

- $0 \leq \alpha \leq 1$ ou equivalentemente $0 \% \leq \alpha \leq 100 \%$;

- ParamRef: parâmetro (latência, vazão ou RREQ) medido na simulação sem a implementação do PWSave, ou seja, resultados do parâmetro com a associação de: AODV + Gossip + Quorum;

- Param: parâmetro (latência, vazão ou RREQ) medido na simulação com a implementação do PWSave;

- ConsumoEnergiaRef: consumo de energia medido na simulação sem a implementação do PWSave;

- ConsumoEnergia: consumo de energia medido na simulação com a implementação do PWSave.

\subsection{Resultados de Simulação}

Nesta seção serão apresentados os resultados dos cenários simulados neste trabalho. 


\subsubsection{Resultados da Implementação do algoritmo Gossip nos protocolos de roteamento DSR e AODV}

Neste trabalho foram medidos para os diferentes cenários de simulações: o número de RREQ na rede, a vazão total em bits/seg, latência total da rede em segundos, consumo total de energia em $\mathrm{mWhr}$ e a porcentagem de perda de pacotes.

Inicialmente foram feitas as simulações com os protocolos de roteamento DSR e AODV sem o algoritmo Gossip e, posteriormente, com a adição deste, para comparar se houve melhora da performance dos parâmetros (RREQ, vazão, latência, perda de pacotes e consumo de energia) com o uso do Gossip. Foram feitas 150 simulações com o DSR para obter uma distribuição Normal das medidas, Figura 4.5, em comparação com as 100 simulações necessárias para o AODV que apresentaram um resultado que seguiam uma distribuição Normal.

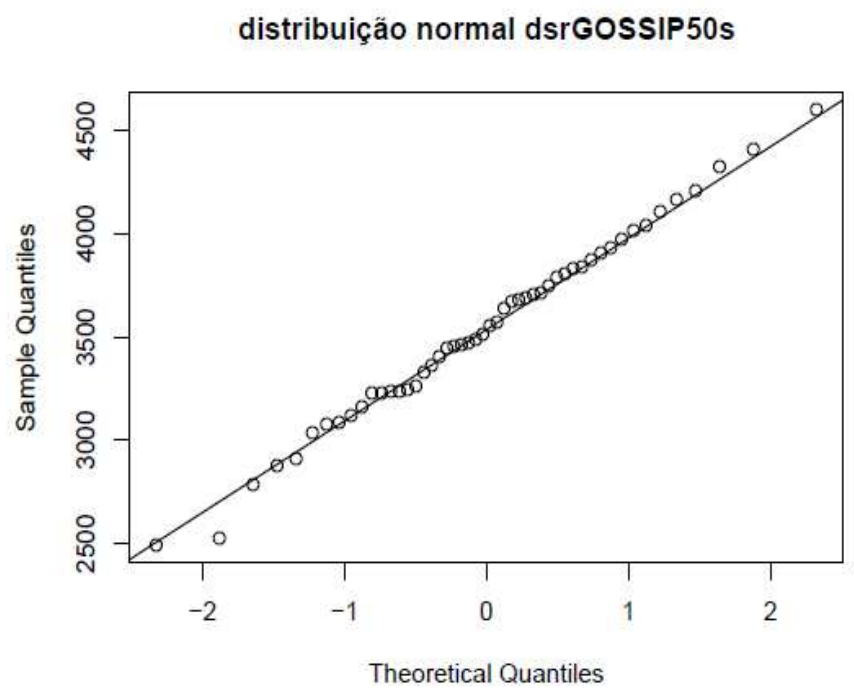

Figura 4.5: Distribuição Normal do DSR com Gossip com tempo de pausa de mobilidade de 50 segundos.

A Figura 4.6 mostra os números de RREQ na rede segundo os diferentes tempos de pausa (50,100, 150, 200, 250 segundos, e o cenário sem mobilidade representado na Figura 4.6 como 300 segundos) para, posteriormente, iniciar a mobilidade. O número de RREQ gerado pelo protocolo AODV é superior ao gerado pelo DSR, pelo fato do AODV possuir um sistema de sinalização periódica na rede por meio de suas mensagens HELLO. Mensagens HELLO garantem ao protocolo AODV a característica de possuir suas rotas sempre atualizadas.

Associando o AODV com o Gossip, o número de RREQ decresce como uma conseqüência direta do número reduzido das mensagens geradas pelo Gossip, 


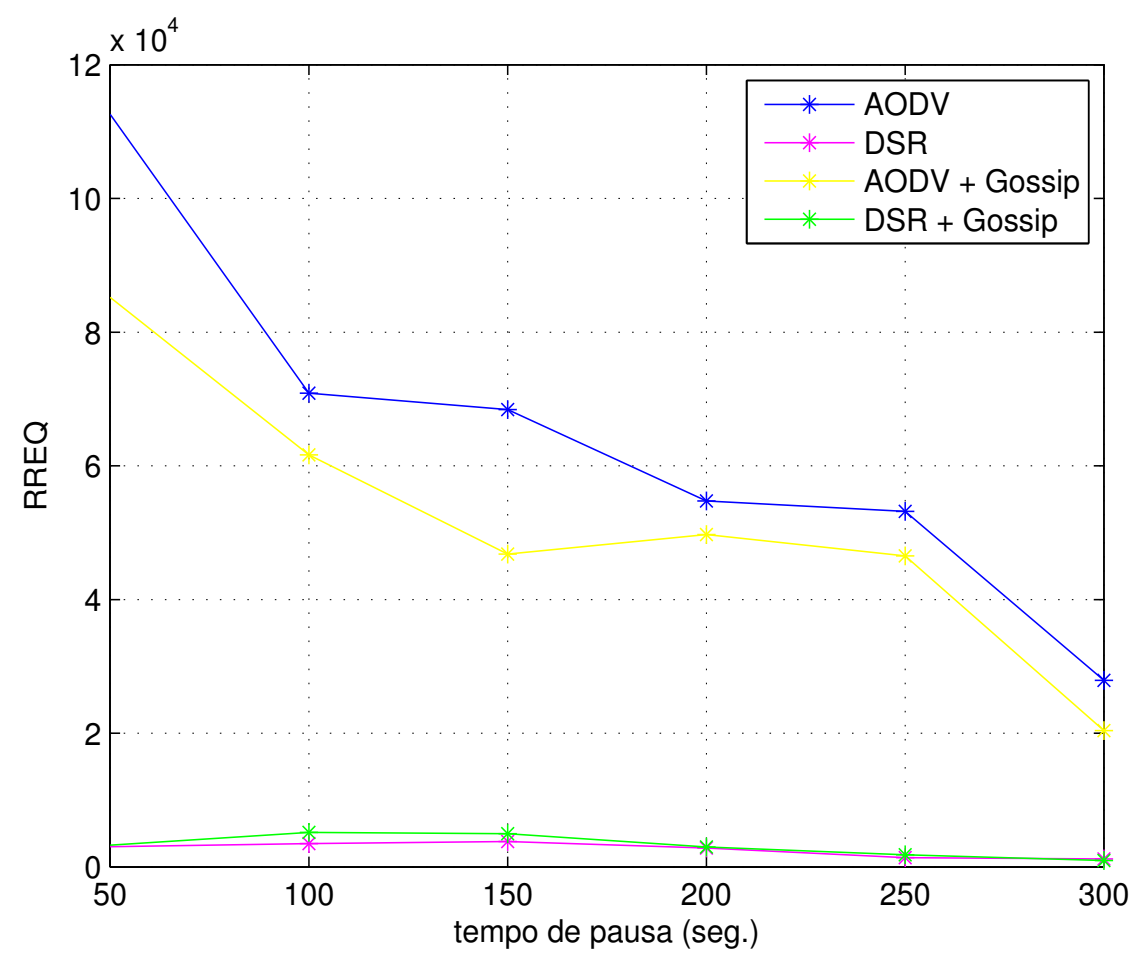

Figura 4.6: Mensagens RREQs para AODV e DSR com e sem a aplicação do algoritmo Gossip em diferentes tempos de pausa.

devido ao seu fator probabilístico usado para decidir para quem devem ser repassadas as informações, evitando o comportamento de flooding. Por outro lado, o DSR apresenta um valor bem baixo de RREQ, devido à sua característica de baixa sinalização, uma vez que o DSR possui um sistema de cache utilizado para conhecer as rotas até um determinado nó, em vez de procurar por rotas mais atuais como é feito no AODV.

Associando o DSR com Gossip nota-se um pequeno aumento do número de RREQ pelo fato de que o Gossip possui um certo grau de redundância no envio de informações.

De acordo com a Figura 4.7, nota-se que o AODV possui uma maior vazão de informações do que o DSR no cenário de mobilidade. Isto ocorre porque o AODV apresenta um esquema de rotas mais atuais e, conseqüentemente, rotas existentes entre os nós. Sem mobilidade, o DSR apresenta uma performance melhor que o AODV devido à sua baixa sobrecarga de atualização de rotas. No cenário de mobilidade, a probabilidade de que a informação armazenada no cache do DSR se torne inválida aumenta, diminuindo a vazão total. A associação do Gossip com ambos os protocolos tem pouca influência em suas performances. Contudo, a suave diminuição da vazão pode ser explicada pelo fato de que o Gossip sacrifica a perda de pacotes para manter uma sobrecarga baixa na rede. 


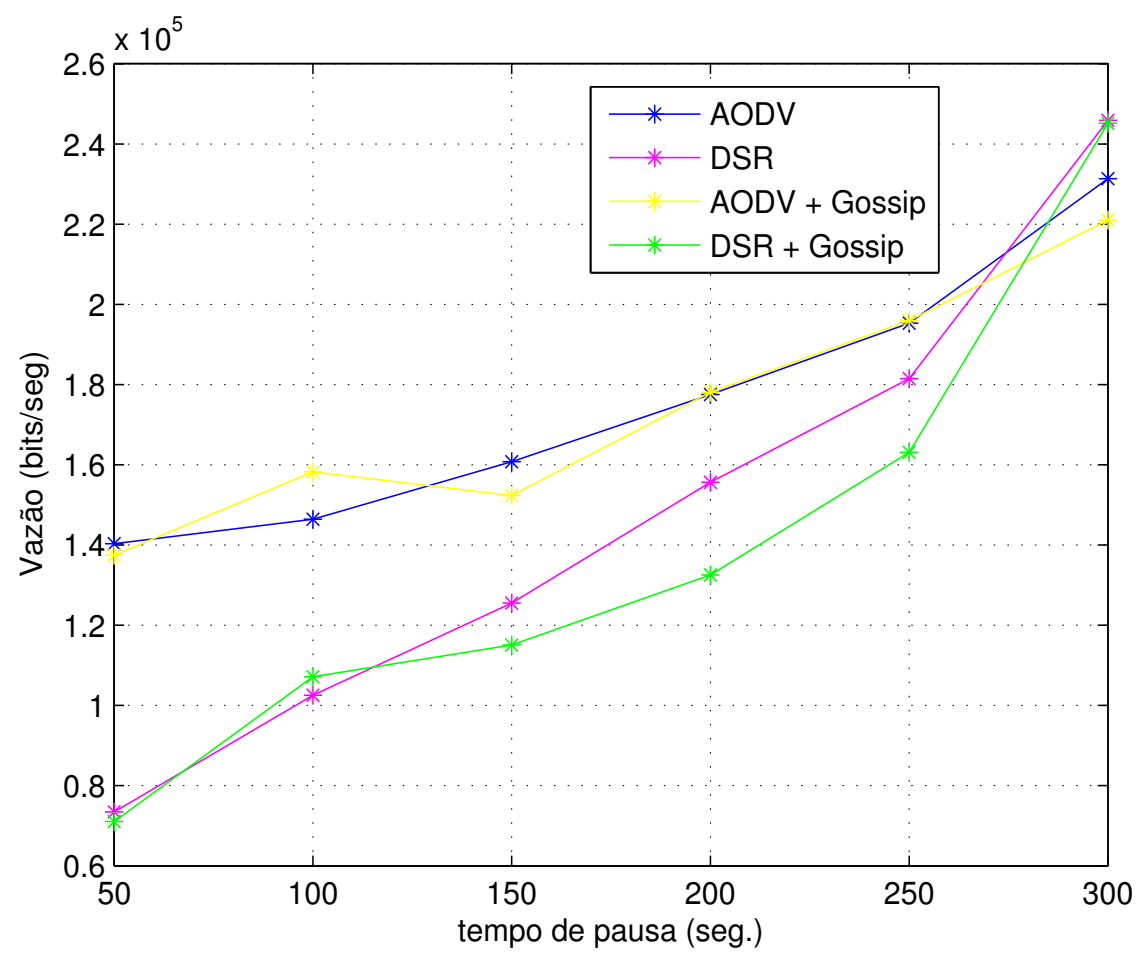

Figura 4.7: Vazão para AODV e DSR com e sem a aplicação do algoritmo Gossip em diferentes tempos de pausa

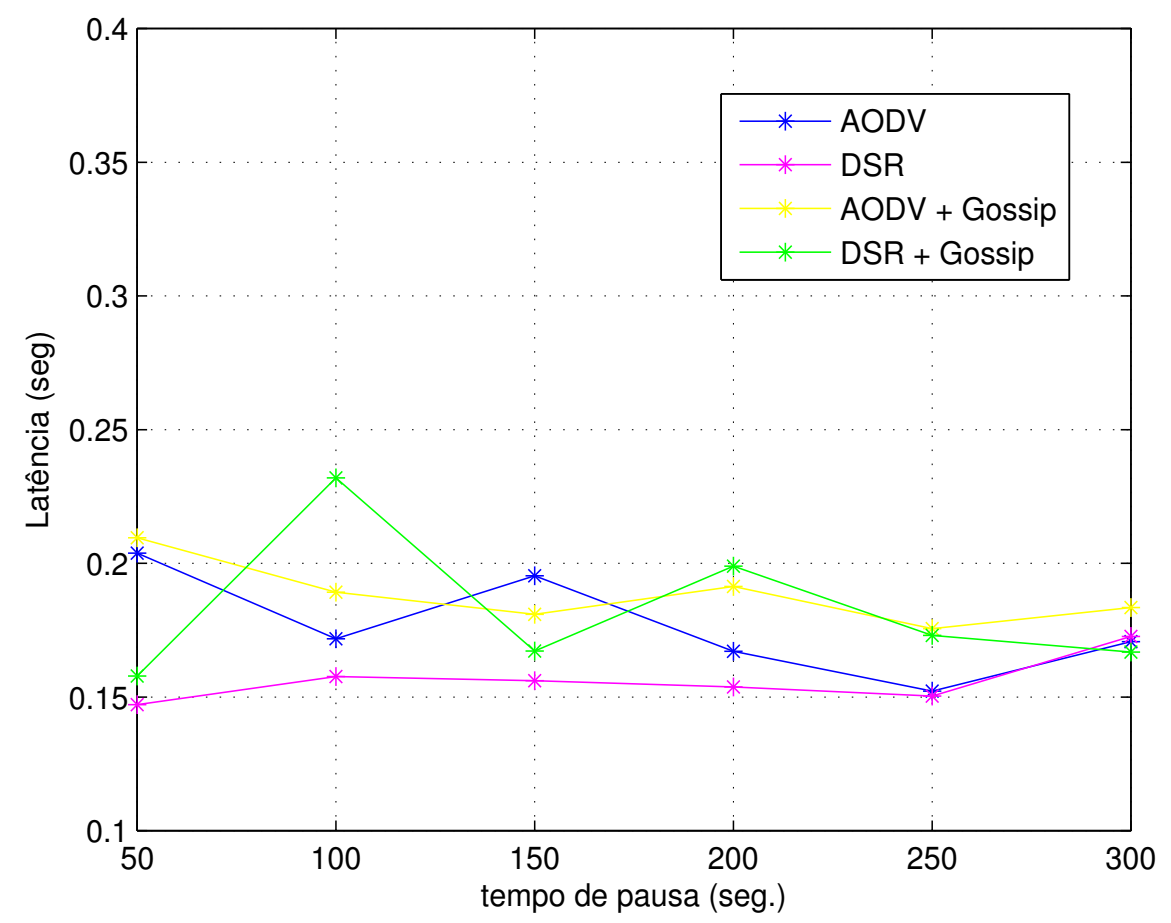

Figura 4.8: Latência para AODV e DSR com e sem a aplicação do algoritmo Gossip em diferentes tempos de pausa

Na Figura 4.8, a performance do DSR, em termos de latência, é melhor que a do AODV. Isto pode ser facilmente explicado porque o DSR utiliza um sistema de cache e o AODV é um protocolo mais reativo, dado que precisa encontrar novas 
rotas enviando RREQ pela rede. Assim, o DSR não tem que validar novas rotas em todo momento e o protocolo AODV gera um atraso causado pela atualização das suas tabelas de roteamento. Associando o Gossip a ambos os protocolos a latência aumenta, porque há um atraso para calcular os vizinhos para os quais ele deve repassar as informações por meio do fator probabilístico, uma vez que não há repasse imediato da informação.

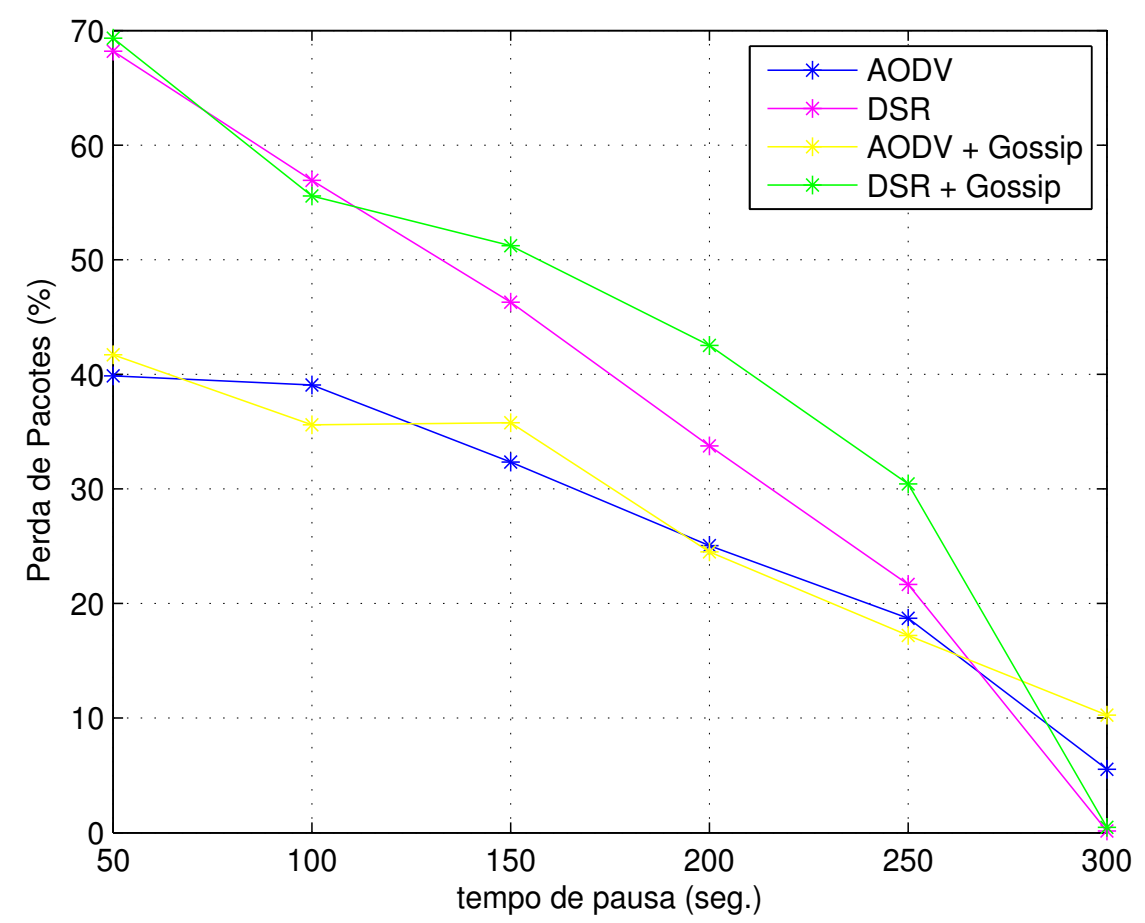

Figura 4.9: Perda de pacotes para AODV e DSR com e sem a aplicação do algoritmo Gossip em diferentes tempos de pausa

A Figura 4.9 mostra a perda de pacotes em valores percentuais: o DSR possui uma perda de pacotes maior que o AODV no cenário de mobilidade devido ao sistema de cache, que pode trabalhar com rotas desatualizadas e, assim, os dados podem não ser entregues se as rotas foram modificadas pelo deslocamento dos nós. O Gossip piora a performance do DSR por adicionar um pequeno atraso na disseminação de informação, o que é prejudicial ao DSR. Quando a mobilidade diminui, a perda de pacotes também diminui, pois o DSR é um protocolo de roteamento que possui boa performance nos cenários estáticos.

Assim, embora o algoritmo Gossip sacrifique algumas características de performance de atraso e vazão, ele diminui o número de mensagens RREQ, que representa um fator de consumo de energia e sobrecarga. De acordo com a Figura 4.10, vê-se que o algoritmo Gossip produz uma redução do consumo de energia. Essa redução observada, embora não seja muito significativa, mostra que tornar o Gossip power-aware pode ser interessante, uma vez que parte dos dispositivos, 


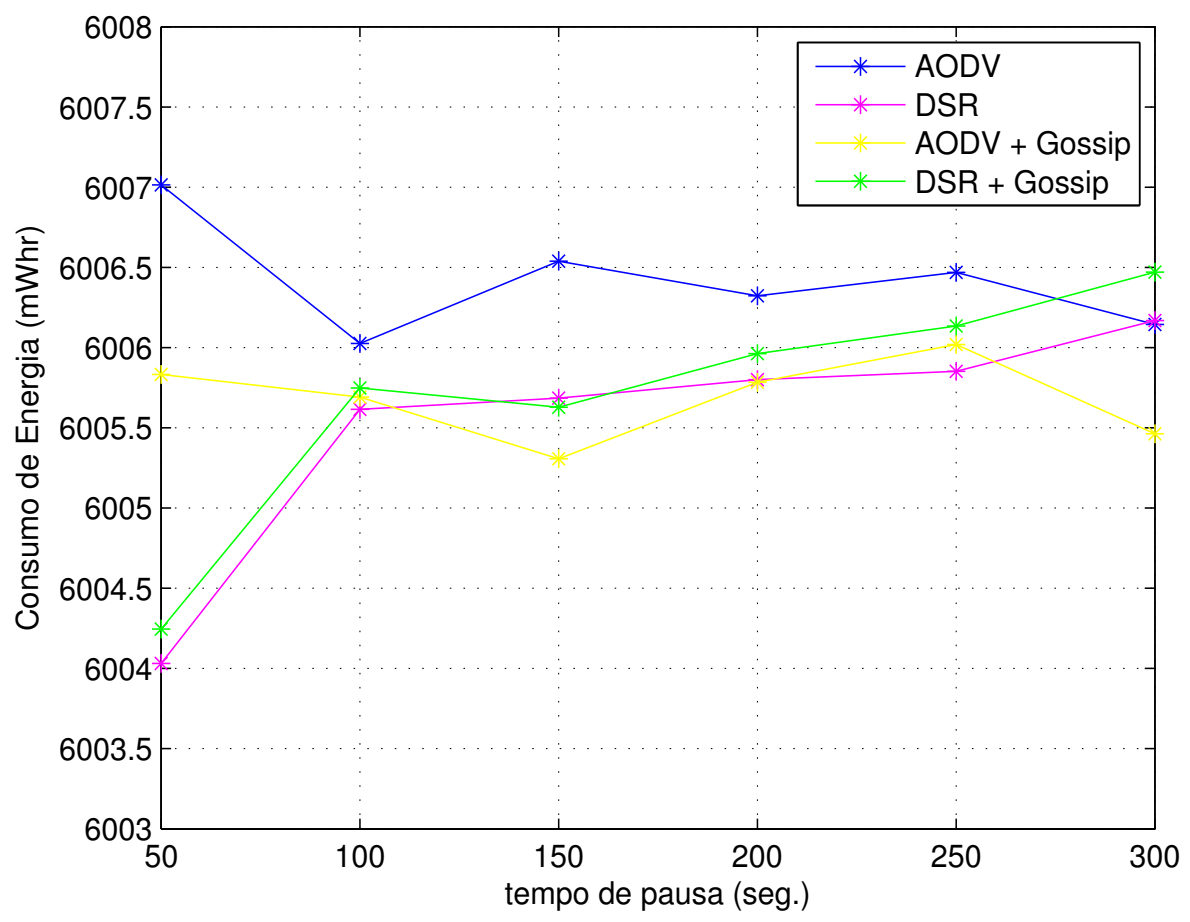

Figura 4.10: Consumo de energia para AODV e DSR com e sem a aplicação do algoritmo Gossip em diferentes tempos de pausa

após aplicar o Gossip, pode passar ao estado idle e aqueles que se encontram neste estado poderiam entrar em um estado sleeping, ocorrendo a economia de energia.

\subsubsection{Resultados da Implementação do algoritmo Gossip e Sistema de Quorum}

As Figuras 4.11 e 4.12 mostram, respectivamente, a perda de pacotes para o AODV e o DSR, com e sem algoritmo Gossip e sistema de Quorum. Verificase que não há modificações relevantes com a adição do sistema de Quorum; os demais parâmetros (RREQ, vazão, latência e consumo de energia) também não apresentam modificaçoes relevantes, se forem comparados com os resultados sem o sistema de Quorum.

O sistema de Quorum causa uma redundância das informações e, em algumas ocasiões, mostra-se útil, como no caso do cenário de falhas, explicado na próxima seção.

Este trabalho verificou que o sistema de Quorum, sem ser somado com um modelo de falhas, em nada altera os resultados dos parâmetros medidos nos vários protocolos estudados. Portanto, como o protocolo DSR no cenário de mobilidade, continua a apresentar $70 \%$ de perda de pacotes e este trabalho abrange os cenários 


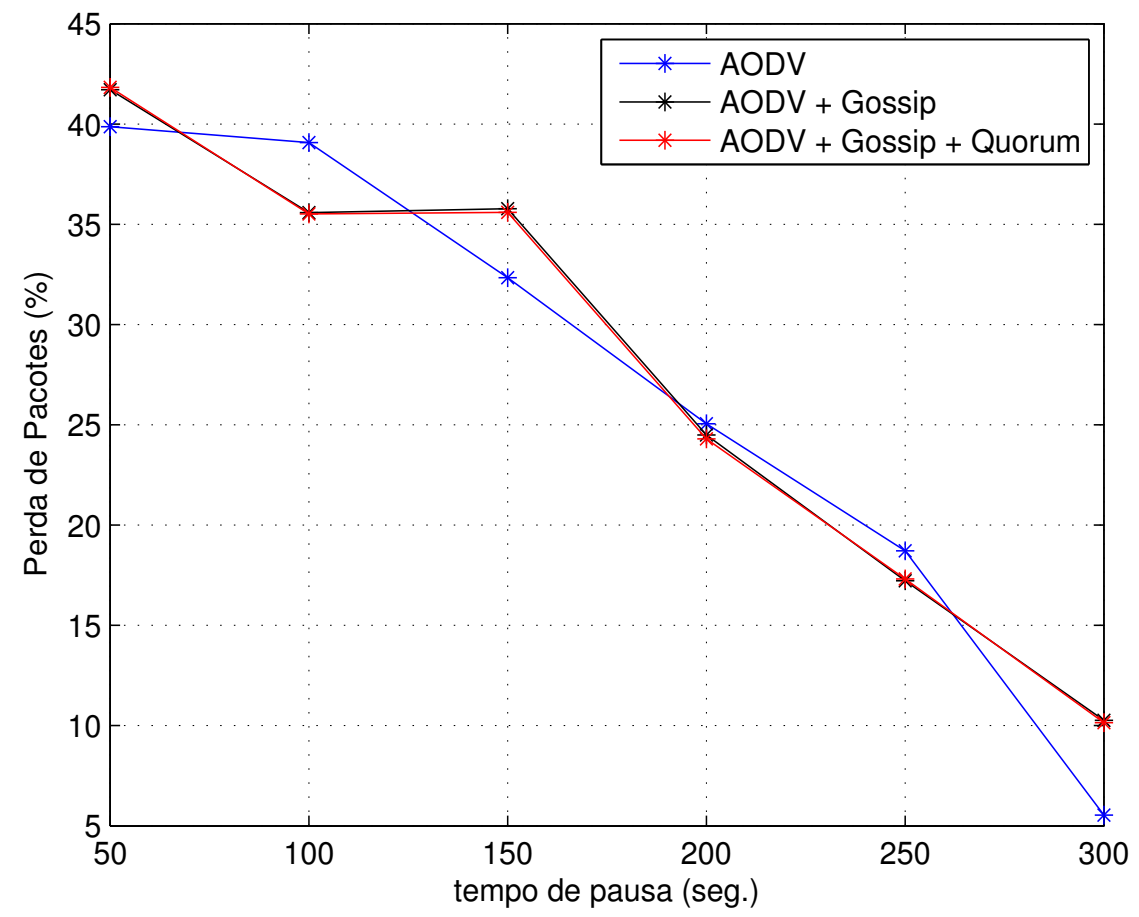

Figura 4.11: Perda de pacotes para AODV com algoritmo Gossip e sistema de Quorum em diferentes tempos de pausa

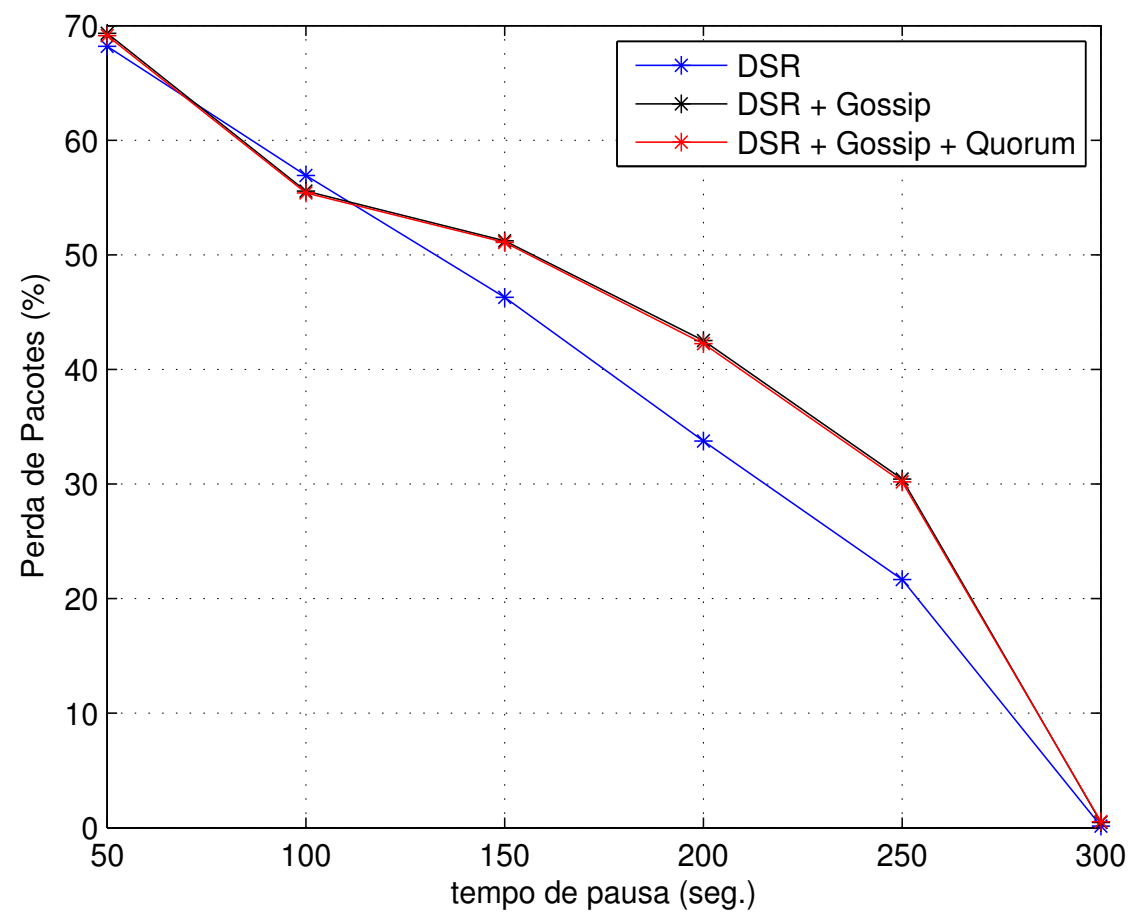

Figura 4.12: Perda de pacotes para DSR com algoritmo Gossip e sistema de Quorum em diferentes tempos de pausa

dinâmicos, a partir dos demais cenários não será feita a simulação do protocolo DSR, senão que será utilizado apenas o protocolo AODV, por ser mais indicado e possuir uma boa performance em um cenário de alta mobilidade. 


\subsubsection{Resultados da Implementação de Falhas}

Foi verificado que o conjunto de simulações apresenta uma regularidade de resultados, conforme visto na Figura 4.13, que se refere à medida do algoritmo Gossip associado ao sistema de Quorum com um tempo de pausa de 150 segundos e uma falha de $30 \%$ do cenário A, para o parâmetro de perda de pacotes. Os demais gráficos não serão apresentados, dado que foi obtido um resultado de normalidade semelhante ao da Figura 4.13.

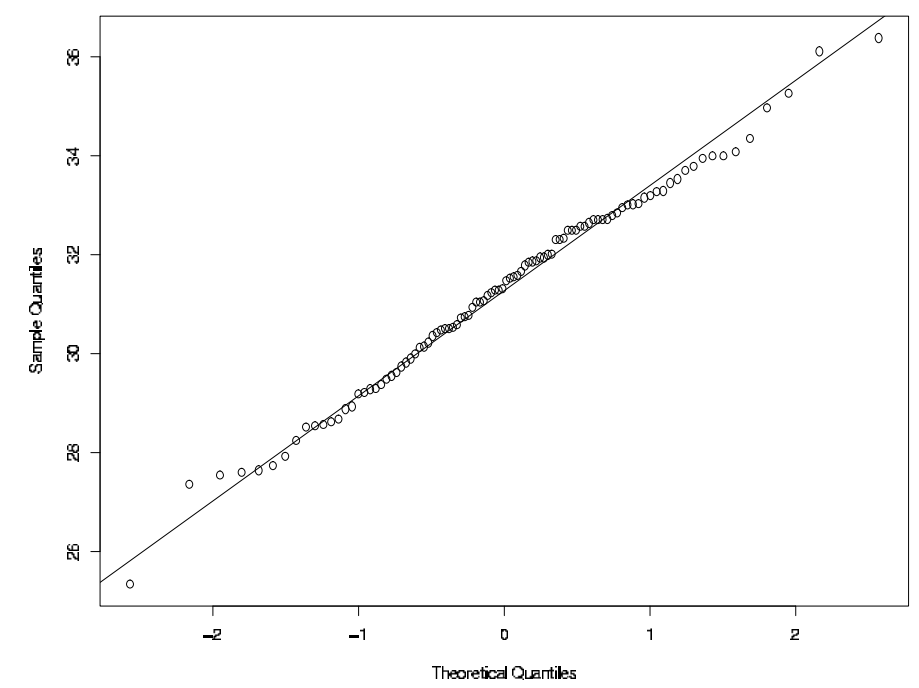

Figura 4.13: Distribuição normal - AODV + Gossip + Quorum com tempo de pausa de 150 seg e falha de $30 \%$.

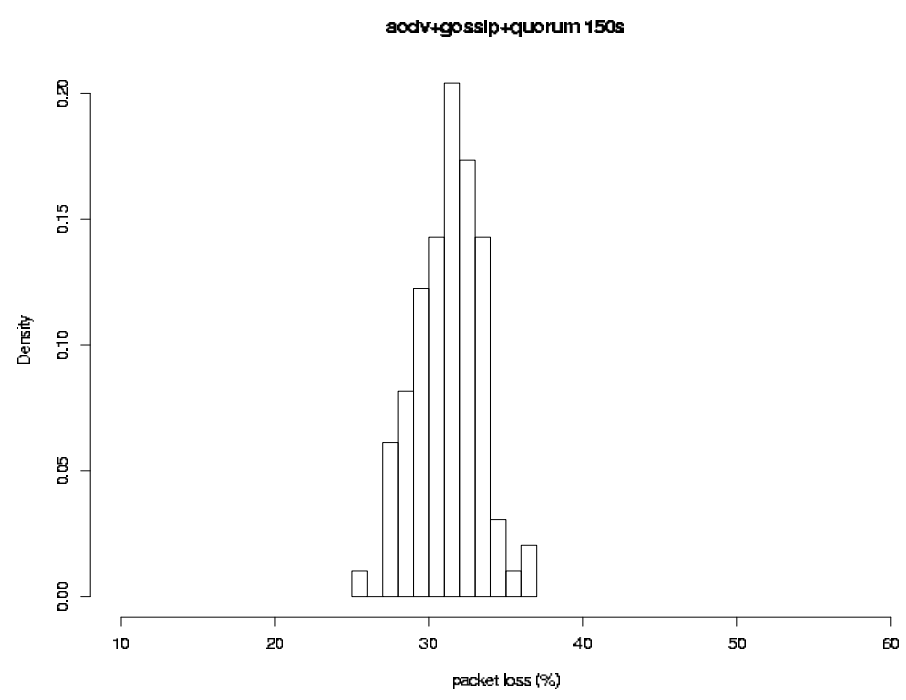

Figura 4.14: Histograma - AODV + Gossip + Quorum com tempo de pausa de 150 segundos e falha de $30 \%$

A Figura 4.14 apresenta o histograma referente à Figura 4.13, que leva a afirmar que os resultados obtidos fazem parte de uma distribuição normal, comprovando sua validade. Os demais histogramas referentes às simulações de falhas, 
tempos de pausa e parâmetros medidos nos cenários A e B apresentam resultados bem semelhantes e, por este motivo, não serão apresentados.

As Figuras de 4.15 a 4.24 apresentam os valores médios, referentes às 100 simulações, dos parâmetros medidos nos cenários A e B. No eixo x são apresentadas as porcentagens de falha $(7,5,15,22,5$ e $30 \%)$ e no eixo y os tempos de pausa de 50, 100, 150, 200, 250 segundos e o cenário sem mobilidade, que é chamado de 300 seg; o eixo z, por sua vez, mostra a performance do parâmetro de interesse.

Foram calculados os desvios padrões para se obter a dispersão dos resultados nas simulações efetuadas e comprovar o desempenho dos algoritmos. Os resultados obtidos foram satisfatórios.

Nota-se que os valores dos parâmetros de latência, Figuras 4.15 e 4.16, não sofreram degradação significativa com a aplicação do Gossip e do Quorum no protocolo de roteamento AODV. O pequeno aumento da latência é explicado pelo fato do algoritmo Gossip causar uma latência ao calcular os nós, aos quais se deve passar as informações, e devido a que o sistema de Quorum causa uma latência ao depositá-las nos servidores do Quorum. E este aumento de latência entre a associação do Gossip e Quorum se torna mais acentuado conforme aumenta a probabilidade de falha dos nós, visto que conforme se reduz o número de nós ativos na rede há um aumento cada vez maior do tempo para levar a informação aos servidores do Quorum.

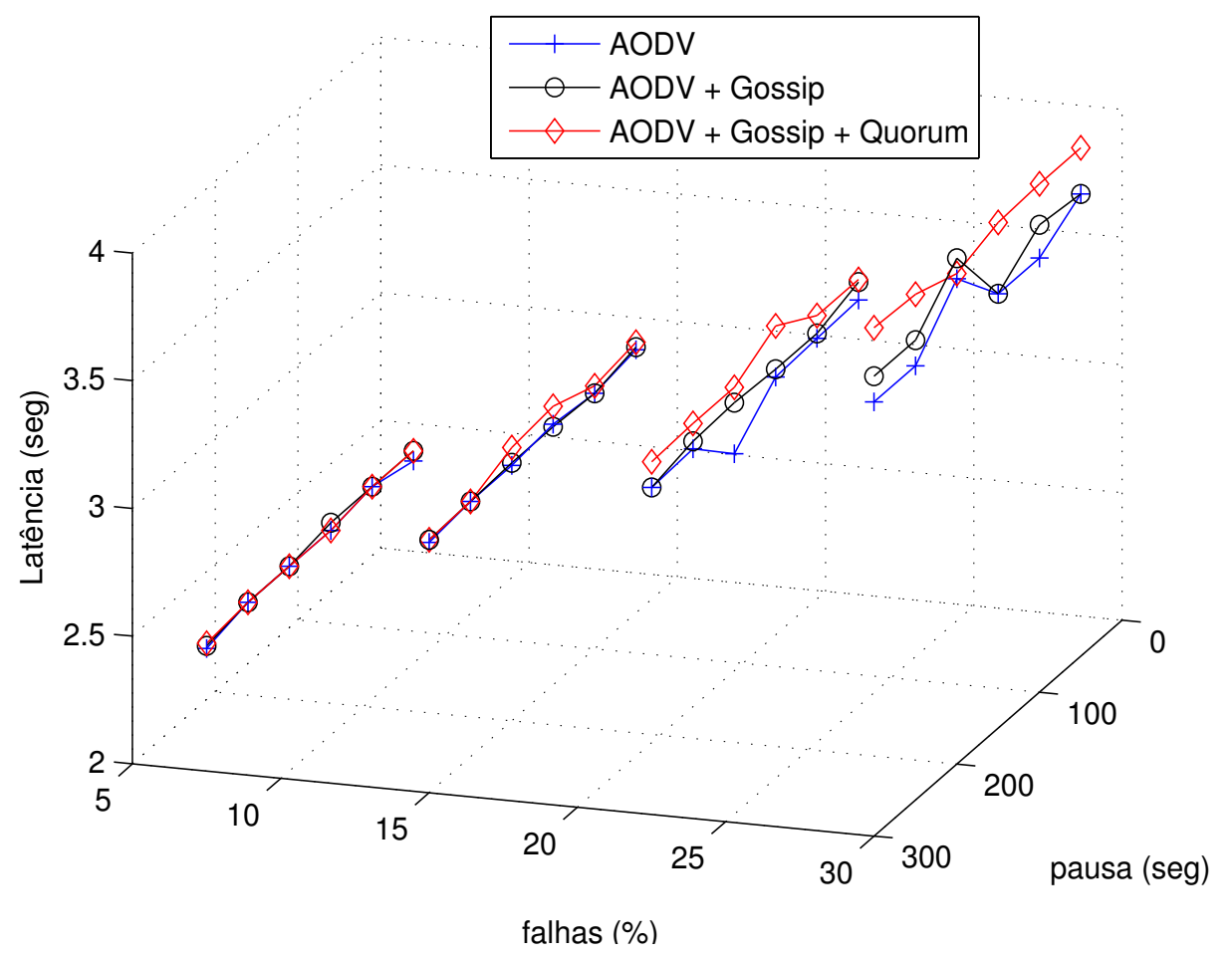

Figura 4.15: Latência - Cenário A 


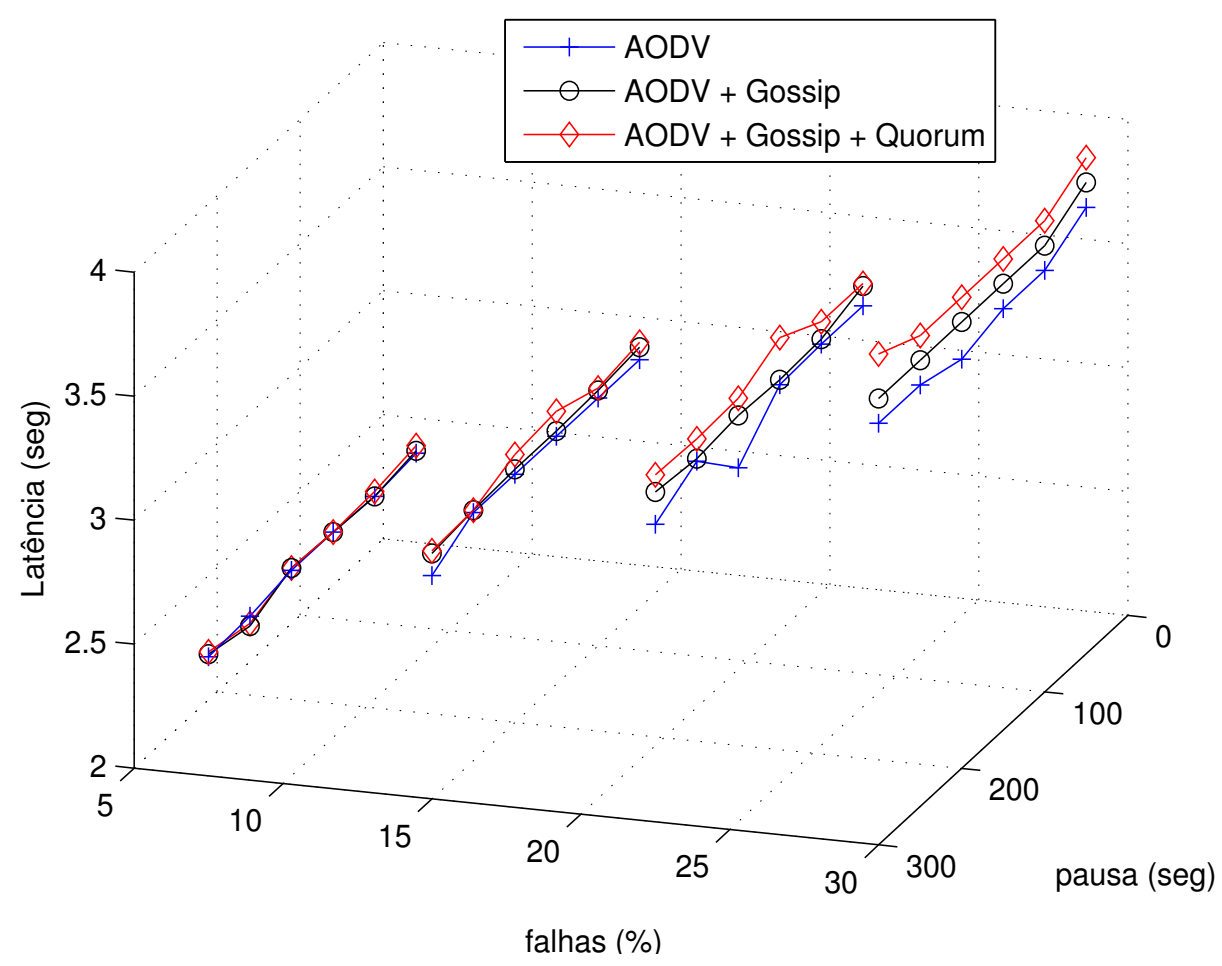

Figura 4.16: Latência - Cenário B

A Tabela 4.2 mostra os valores médios e desvios padrões da latência do cenário A. Na sequência serão mostrados somente os gráficos. As tabelas referentes aos resultados obtidos são apresentados no Anexo B.

Por simplificação, nas tabelas foi utilizada a sigla $\mathrm{AODV}+\mathrm{G}$, significando $\mathrm{AODV}+$ Gossip e AODV $+\mathrm{G}+\mathrm{Q}$, significando AODV + Gossip + Quorum.

Nas Figuras 4.17 e 4.18 nota-se que há um aumento da vazão com a aplicação do sistema de Quorum; contudo, a associação do algoritmo Gossip, sem o uso do sistema de Quorum, não causa nenhum benefício aos resultados da vazão. Isso pode ser explicado pelo fato do sistema de Quorum guardar uma parte dos dados em seus servidores de dados, permitindo que os nós que queiram ter acesso a estas informações recorram a seus servidores, tornando a entrega de dados mais eficiente.

Os valores de RREQ mostrados nas Figuras 4.19 e 4.20 indicam que com a aplicação do algoritmo Gossip houve uma diminuição significativa em seus valores. Isso deve-se ao fato de que a inundação (flooding) na rede não está mais ocorrendo, uma vez que o algoritmo Gossip envia RREQ somente a alguns nós da rede. Com a aplicação do Quorum há um pequeno aumento de RREQ devido ao repasse das informações para os servidores do Quorum às requisições de informações dos nós clientes; contudo, o aumento de RREQ não é significativo, como pode ser visto nas Figuras 4.19 e 4.20 . 
Tabela 4.2: Valores médios e desvios padrões para latência do Cenário A em segundos

\begin{tabular}{ccccc}
\hline Falhas $(\%)$ & $\begin{array}{c}\text { Tempo de } \\
\text { pausa }(\mathrm{s})\end{array}$ & AODV & AODV $+\mathrm{G}$ & AODV $+\mathrm{G}+\mathrm{Q}$ \\
\hline 7,5 & 0 & $2,45 \pm 0,05$ & $2,46 \pm 0,04$ & $2,47 \pm 0,04$ \\
7,5 & 50 & $2,46 \pm 0,06$ & $2,49 \pm 0,07$ & $2,49 \pm 0,04$ \\
7,5 & 100 & $2,46 \pm 0,04$ & $2,49 \pm 0,04$ & $2,49 \pm 0,05$ \\
7,5 & 150 & $2,47 \pm 0,05$ & $2,49 \pm 0,04$ & $2,47 \pm 0,05$ \\
7,5 & 200 & $2,47 \pm 0,06$ & $2,49 \pm 0,04$ & $2,47 \pm 0,06$ \\
7,5 & 250 & $2,47 \pm 0,04$ & $2,49 \pm 0,05$ & $2,47 \pm 0,07$ \\
15 & 0 & $2,87 \pm 0,06$ & $2,88 \pm 0,05$ & $2,88 \pm 0,04$ \\
15 & 50 & $2,92 \pm 0,04$ & $2,93 \pm 0,05$ & $2,95 \pm 0,04$ \\
15 & 100 & $2,89 \pm 0,05$ & $2,90 \pm 0,06$ & $2,93 \pm 0,06$ \\
15 & 150 & $2,91 \pm 0,07$ & $2,90 \pm 0,06$ & $2,98 \pm 0,04$ \\
15 & 200 & $2,89 \pm 0,05$ & $2,88 \pm 0,05$ & $2,96 \pm 0,07$ \\
15 & 250 & $2,86 \pm 0,06$ & $2,86 \pm 0,07$ & $2,89 \pm 0,06$ \\
22,5 & 0 & $3,28 \pm 0,04$ & $3,28 \pm 0,04$ & $3,38 \pm 0,05$ \\
22,5 & 50 & $3,31 \pm 0,05$ & $3,38 \pm 0,05$ & $3,39 \pm 0,05$ \\
22,5 & 100 & $3,31 \pm 0,06$ & $3,37 \pm 0,07$ & $3,39 \pm 0,06$ \\
22,5 & 150 & $3,29 \pm 0,07$ & $3,32 \pm 0,05$ & $3,47 \pm 0,04$ \\
22,5 & 200 & $3,20 \pm 0,06$ & $3,27 \pm 0,05$ & $3,30 \pm 0,06$ \\
22,5 & 250 & $3,24 \pm 0,06$ & $3,27 \pm 0,04$ & $3,36 \pm 0,07$ \\
30 & 0 & $3,74 \pm 0,04$ & $3,80 \pm 0,04$ & $3,95 \pm 0,04$ \\
30 & 50 & $3,81 \pm 0,05$ & $3,81 \pm 0,04$ & $3,95 \pm 0,04$ \\
30 & 100 & $3,81 \pm 0,04$ & $3,81 \pm 0,04$ & $3,95 \pm 0,07$ \\
30 & 150 & $3,70 \pm 0,04$ & $3,70 \pm 0,04$ & $3,94 \pm 0,04$ \\
30 & 200 & $3,64 \pm 0,05$ & $3,93 \pm 0,04$ & $3,92 \pm 0,06$ \\
30 & 250 & $3,69 \pm 0,05$ & $3,70 \pm 0,04$ & $3,97 \pm 0,06$ \\
\hline
\end{tabular}

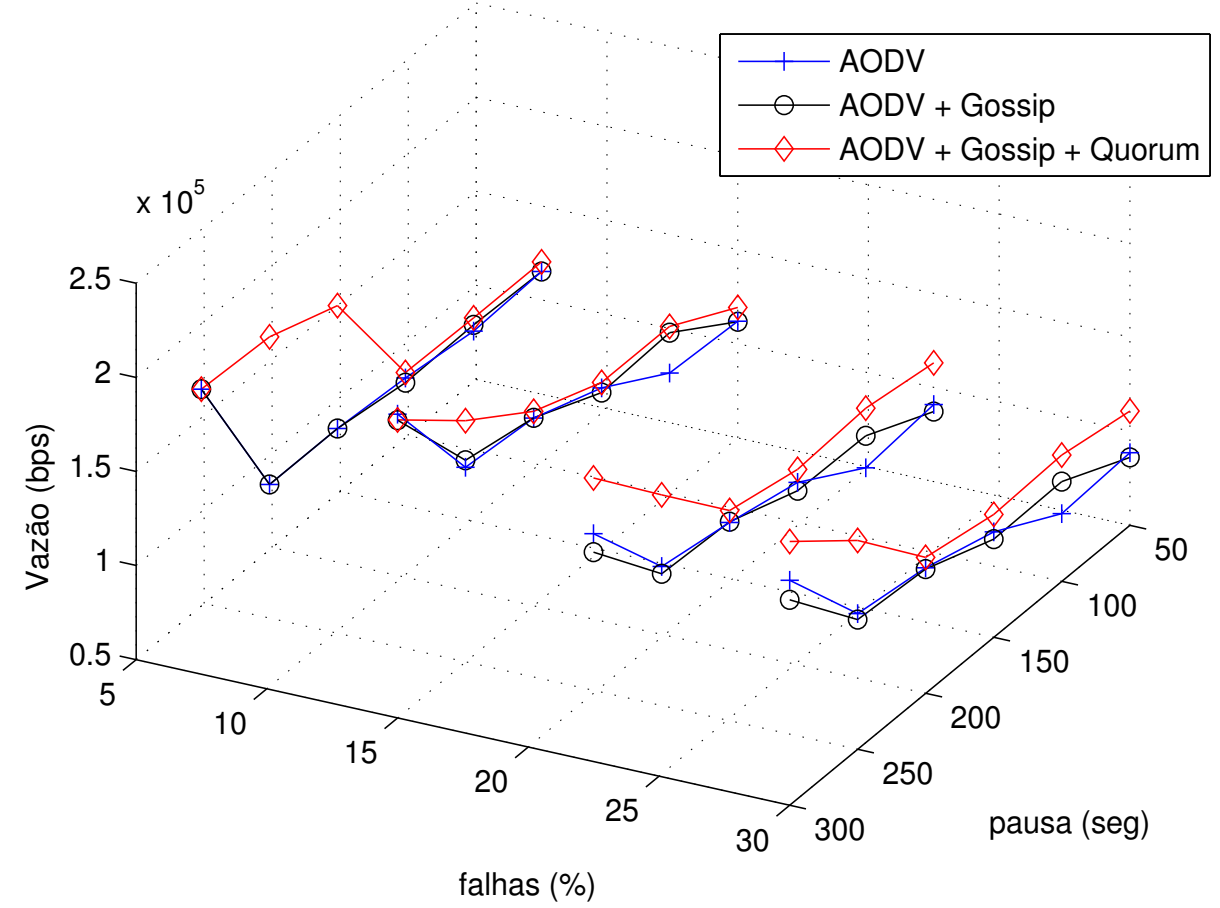

Figura 4.17: Vazão - Cenário A 


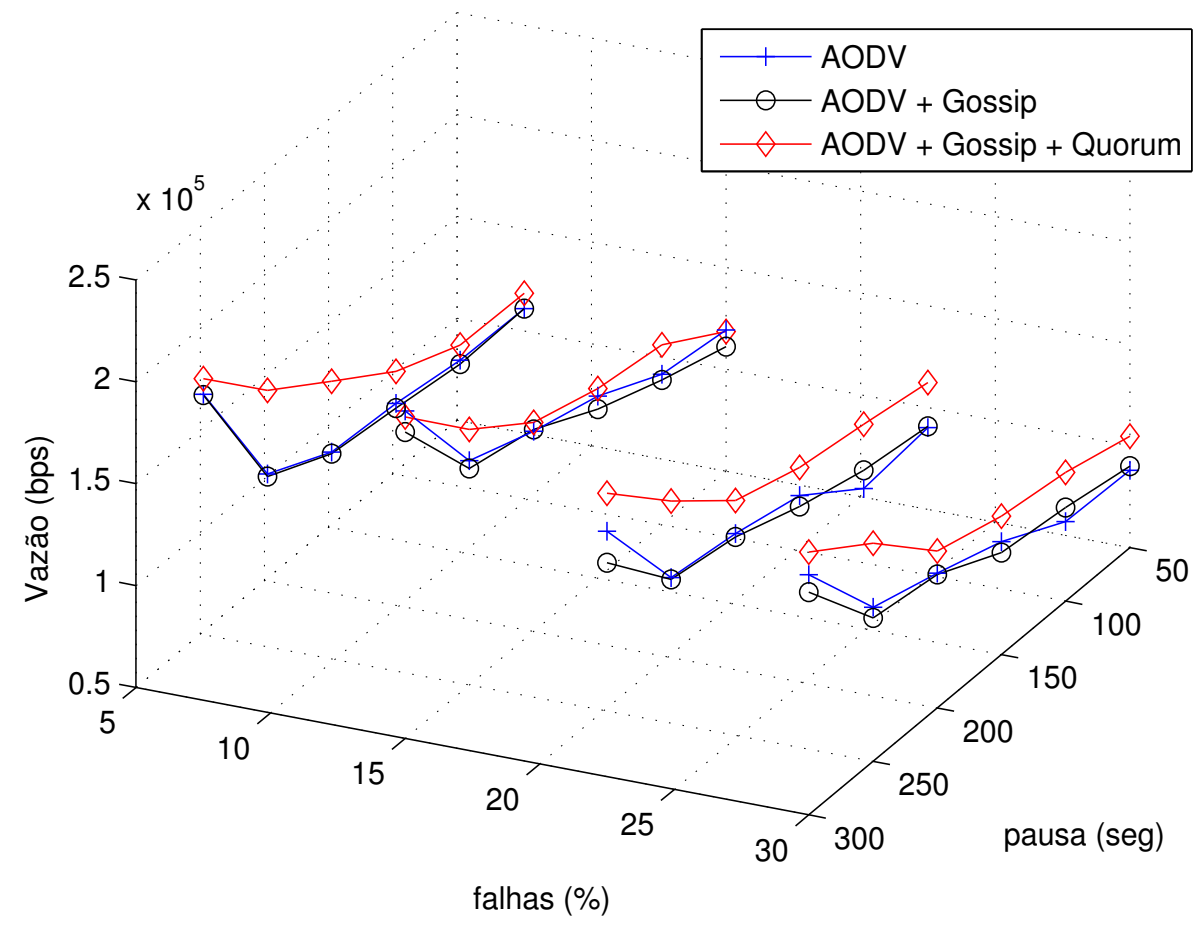

Figura 4.18: Vazão - Cenário B

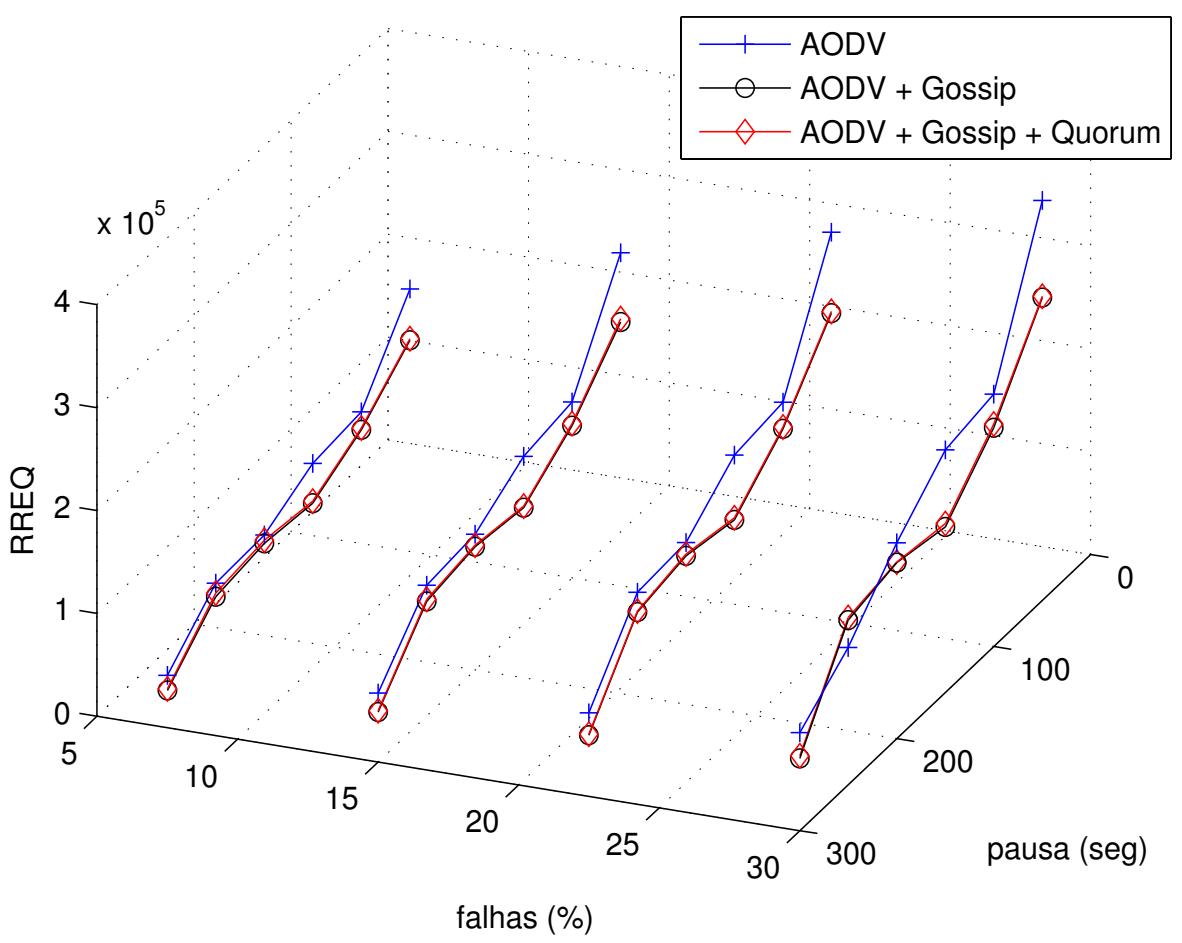

Figura 4.19: RREQ - Cenário A

A variação dos valores de consumo de energia não é significativa, como pode ser visto nas Figuras 4.21 e 4.22, mas deve ser destacado que embora a diferença com e sem a aplicação do algoritmo Gossip não seja significativa, a pequena redução do consumo de energia causada pelo sistema Gossip no protocolo AODV 


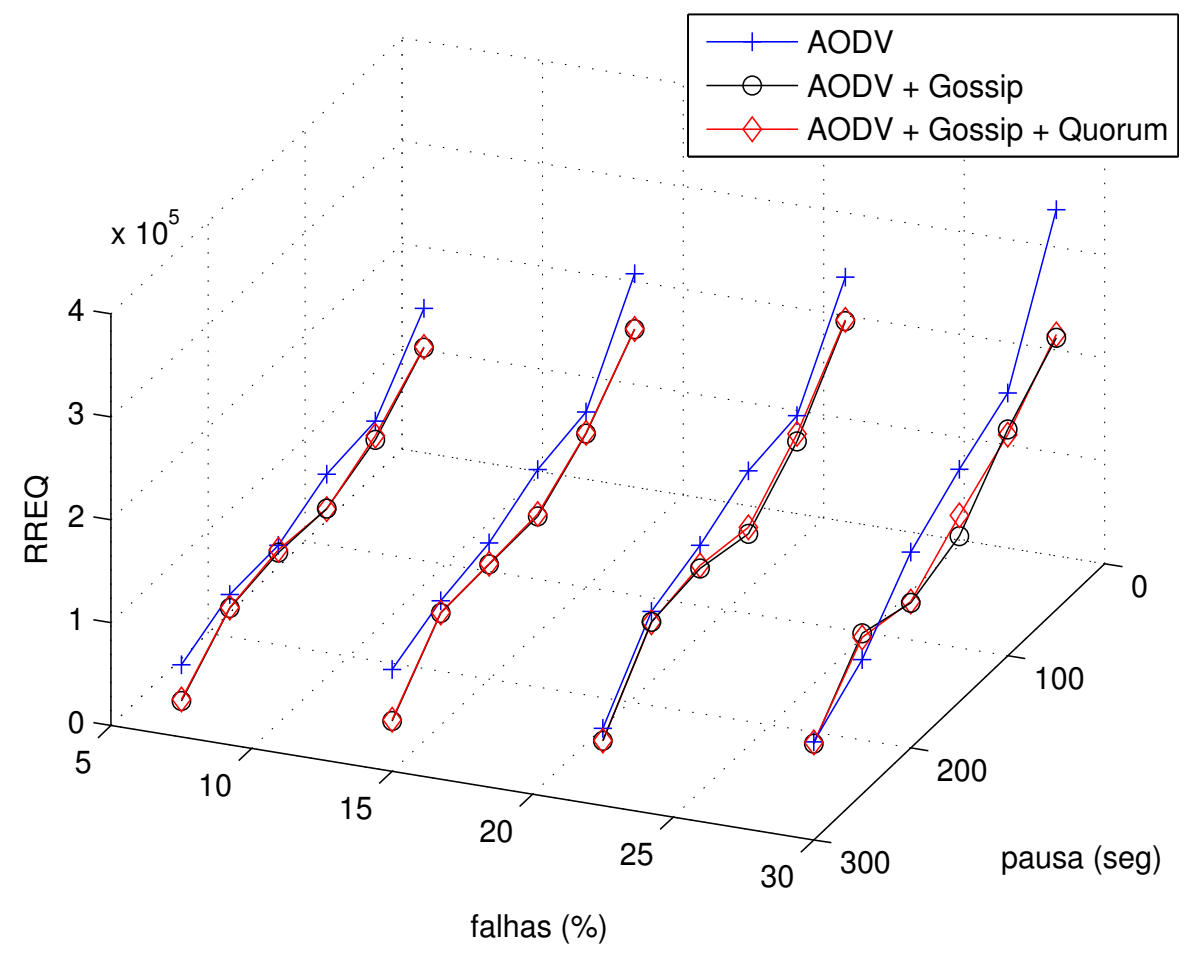

Figura 4.20: RREQ - Cenário B

mostra que se o algoritmo Gossip for melhor trabalhado ele pode apresentar características mais importantes de redução de consumo de energia.

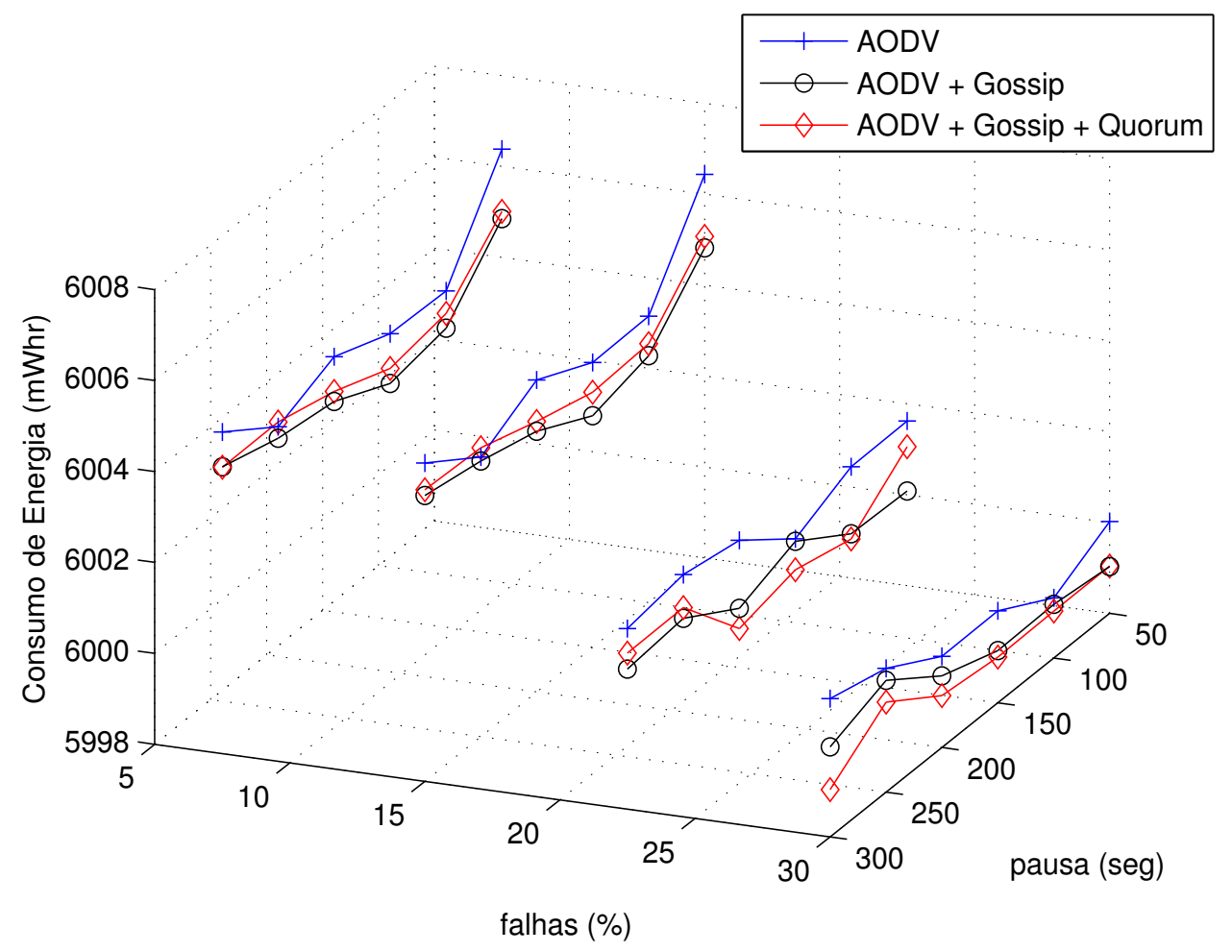

Figura 4.21: Consumo de Energia - Cenário A

O parâmetro de perda de pacotes, Figuras 4.23 e 4.24, não apresenta nenhum benefício apenas com a associação do algoritmo Gossip, mas aplicando o sistema 


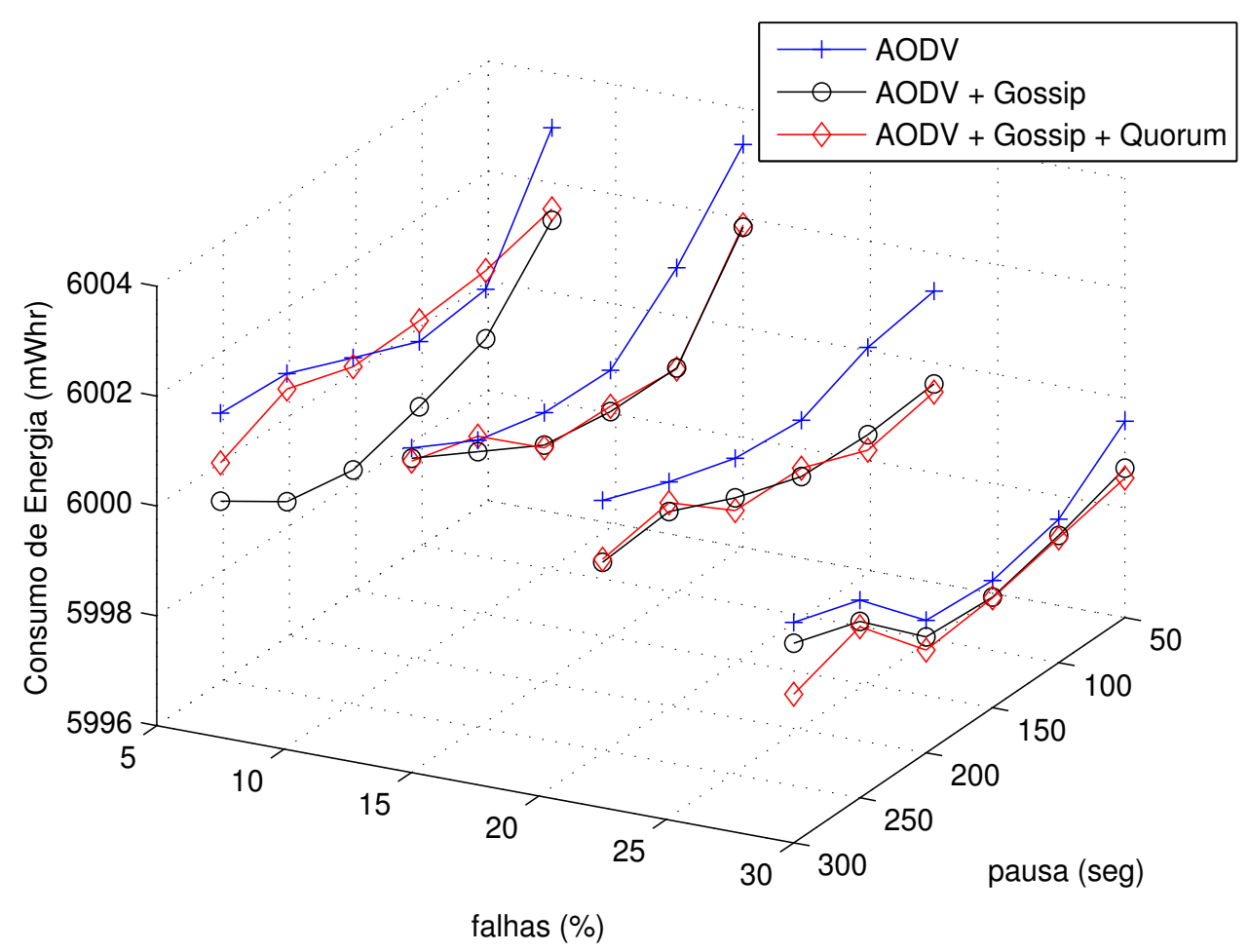

Figura 4.22: Consumo de Energia - Cenário B

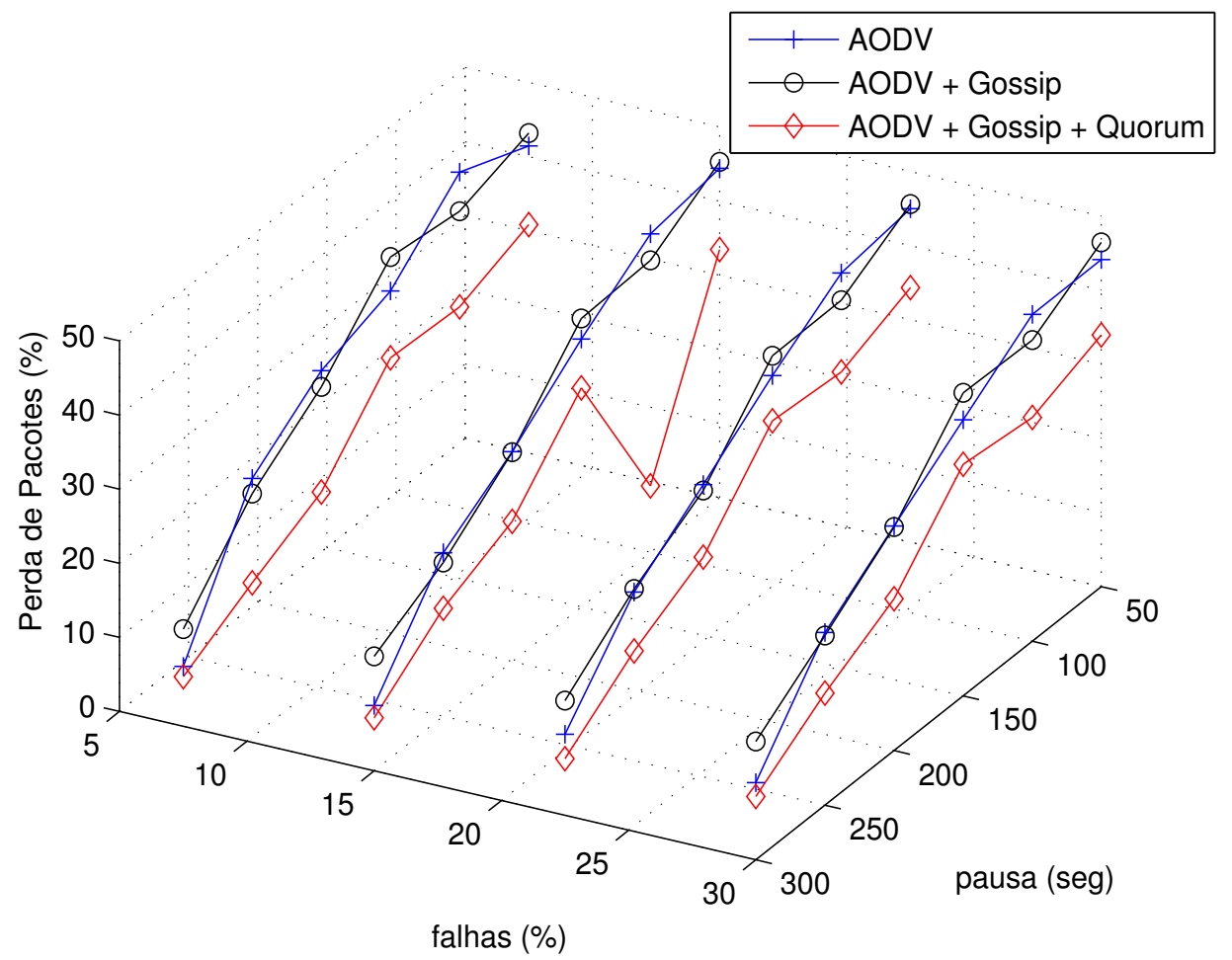

Figura 4.23: Perda de pacotes - Cenário A

de Quorum associado à aplicação de falhas há um decréscimo significativo, que é explicado pelo sistema de distribuição e pela duplicação dos dados em que, caso algum pacote seja perdido, pode haver uma cópia deste dado no servidor do Quorum e este repassa a informação aos clientes solicitantes do pacote requerido. 


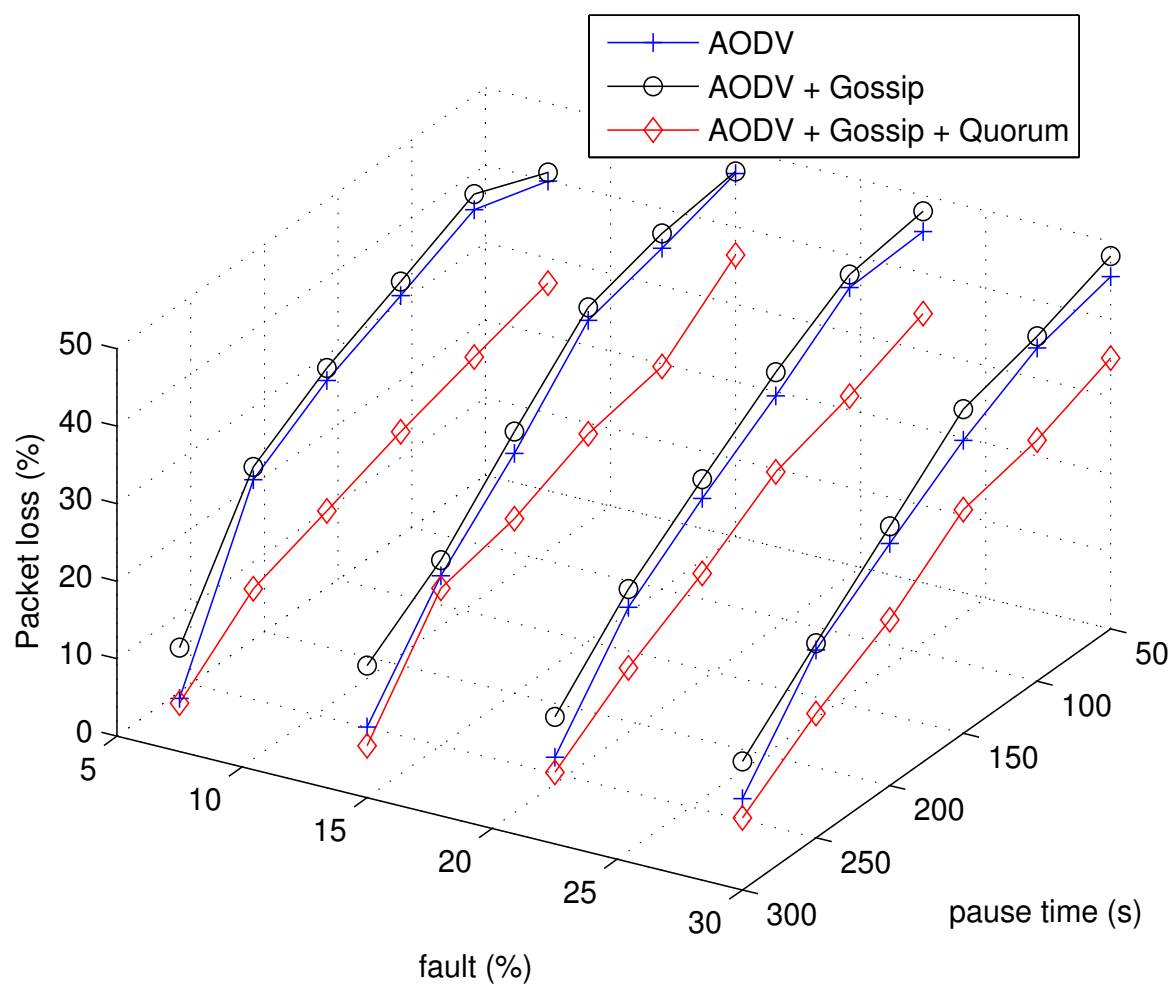

Figura 4.24: Perda de pacotes - Cenário B

Este benefício só é obtido após a aplicação de falhas, dado que após as falhas, os nós fontes já disseminaram as informações aos servidores do Quorum e esta informação pode ser repassada aos seus destinos. Caso haja perda de pacotes e as informações não tenham conseguido chegar até os servidores do Quorum, as perdas de pacotes continuarão a ocorrer.

Os valores obtidos de ambos os cenários apresentam um comportamento similar, o que serve para a validação da associação do algoritmo Gossip com o sistema de Quorum.

Foi verificado nos resultados um decréscimo de $10 \%$ na perda de pacotes em relação à não aplicação do Quorum.

\subsubsection{Resultados da Implementação do algoritmo PWSave}

Nesta seção serão apresentados somente os gráficos referentes ao cenário B, pois conforme pode ser visto nos resultados das tabelas do Anexo B, ambos os cenários, A e B possuem resultados similares.

A Figura 4.25 compara os resultados da perda de pacotes do cenário de simulação com Gossip e Quorum, por simplificação nomeado de AODV $+\mathrm{G}+\mathrm{Q}$, com e sem o algoritmo PWSave no cenário B de falha. Nota-se que há um aumento da taxa de perda de pacotes com a adição do PWSave, pois com o adormecimento 
dos nós a perda de pacotes tende a aumentar, mas a Figura 4.26 mostra que o índice de mérito que calcula o desempenho do parâmetro de perda de pacotes em relação ao consumo de energia, representado em valores percentuais, de 50\%, $75 \%$ e $25 \%$ com falhas a $7,5 \%$ é superior a 1 , ou seja, a economia de energia ainda é vantajosa.

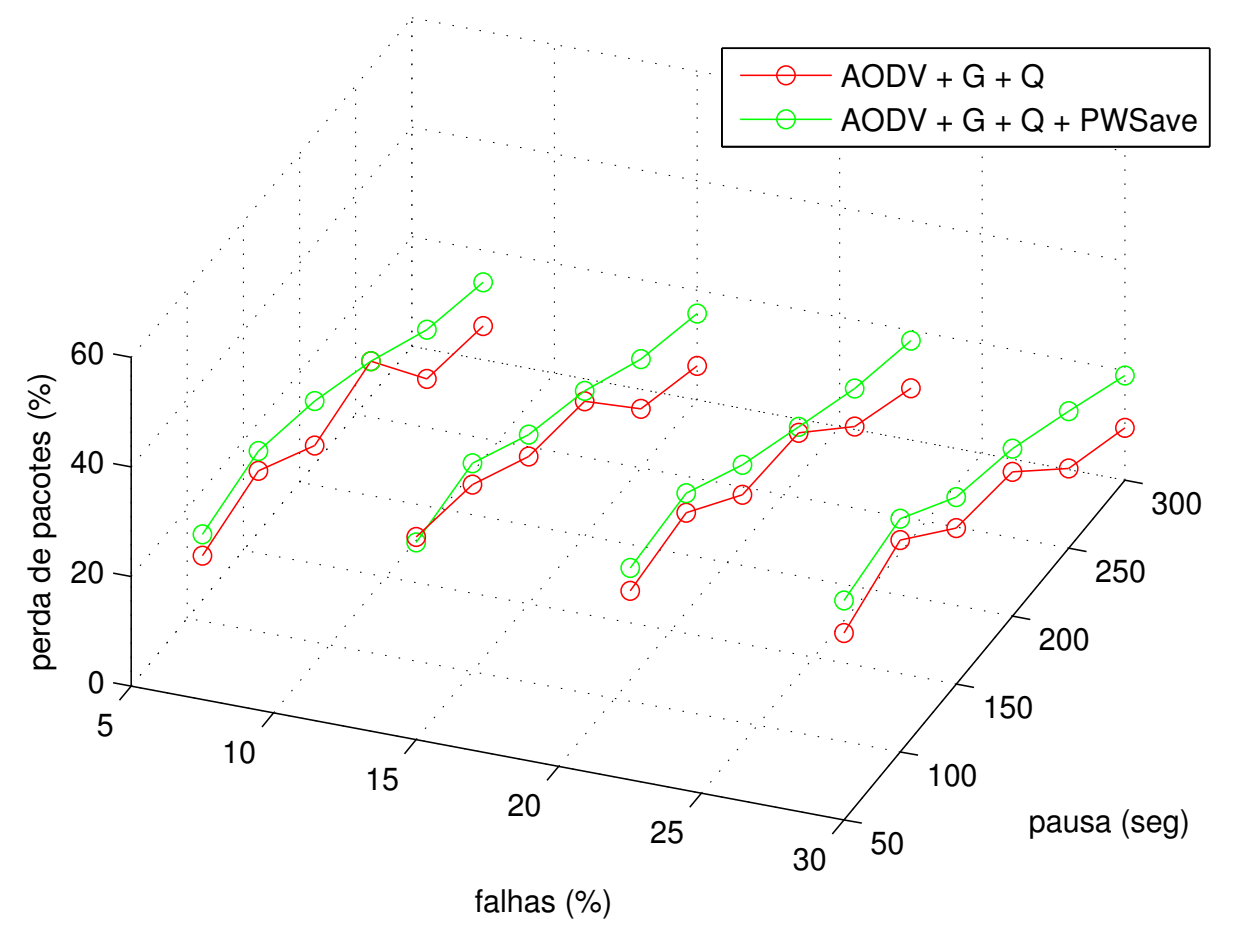

Figura 4.25: Perda de pacotes

A Figura 4.27 compara os resultados da latência dos cenários com Gossip e Quorum com e sem o algoritmo PWSave. Há um aumento da latência com a adição do $P W S a v e$, pois para despertar os nós, quando estes necessitem receber dados ou descobrir novas rotas, é adicionado um atraso na rede.

A Figura 4.28 compara os resultados da vazão dos cenários com Gossip e Quorum com e sem o algoritmo PWSave. Há uma diminuição da vazão com a adição do $P$ WSave, pois com o aumento da perda de pacotes a vazão tende a diminuir.

A Figura 4.29 compara os resultados de RREQ dos cenários com Gossip e Quorum com e sem o algoritmo PWSave. Há um aumento no número de mensagens RREQ com a adição do PWSave, devido à necessidade de gerar novas rotas com o adormecimento de determinados nós, mas a Figura 4.26 mostra que o índice de mérito do RREQ calculado em relação ao consumo de energia é superior a 1 em torno dos 3\%, ou seja, o número de RREQ não é sacrificado com a adição do PWSave. 


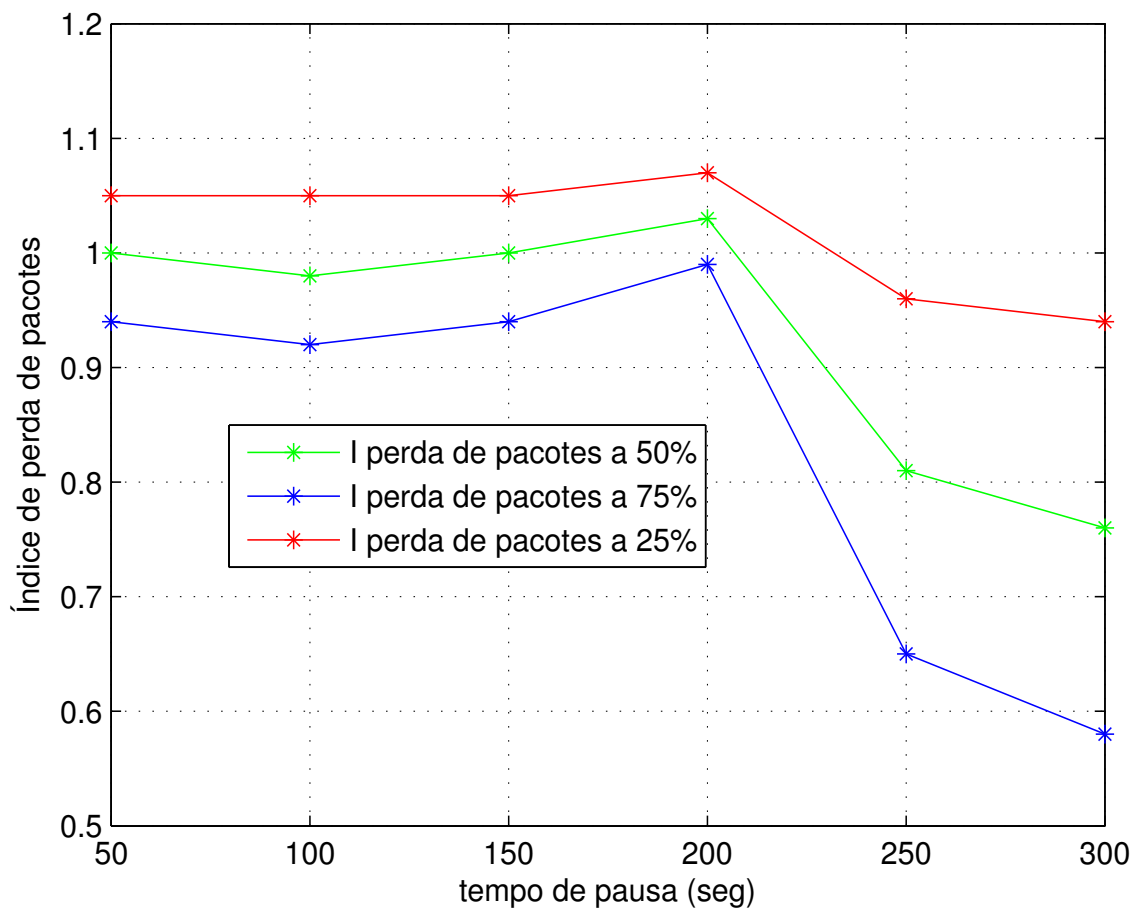

Figura 4.26: Índices de mérito de perda de pacotes a 7,5\% de falha com porcentagens de 50, 75 e 25\% no índice de mérito - Cenário B

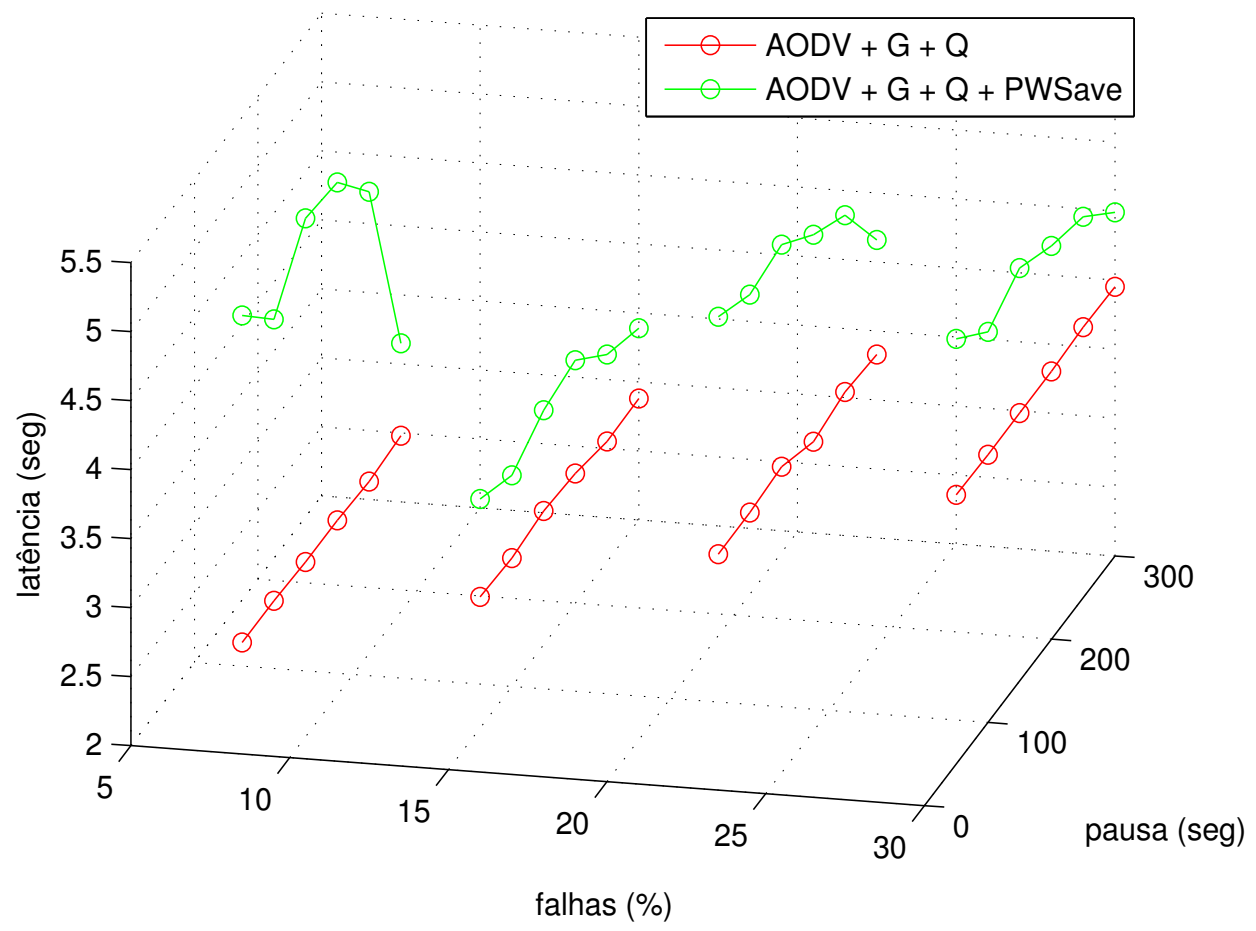

Figura 4.27: Latência

As Figuras 4.30, 4.31, 4.32 e 4.33 mostram os índices de mérito considerando $50 \%$ do parâmetro em específico (perda de pacotes, vazão, latência e RREQ) com o parâmetro de consumo de energia de 50\% no cenário B. O cenário A não será mostrado, pois apresenta índices de mérito similares aos calculados no cenário B. 


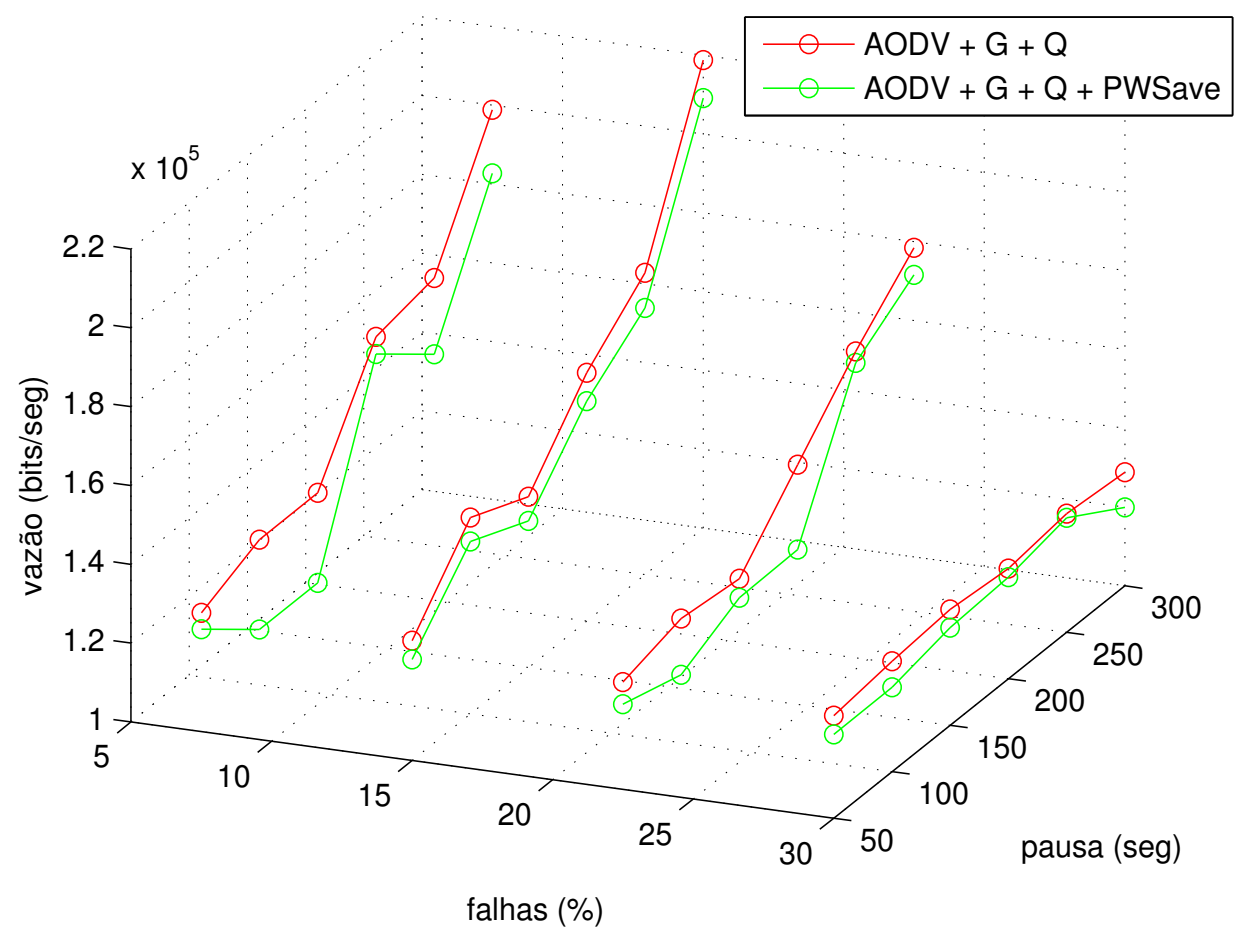

Figura 4.28: Vazão

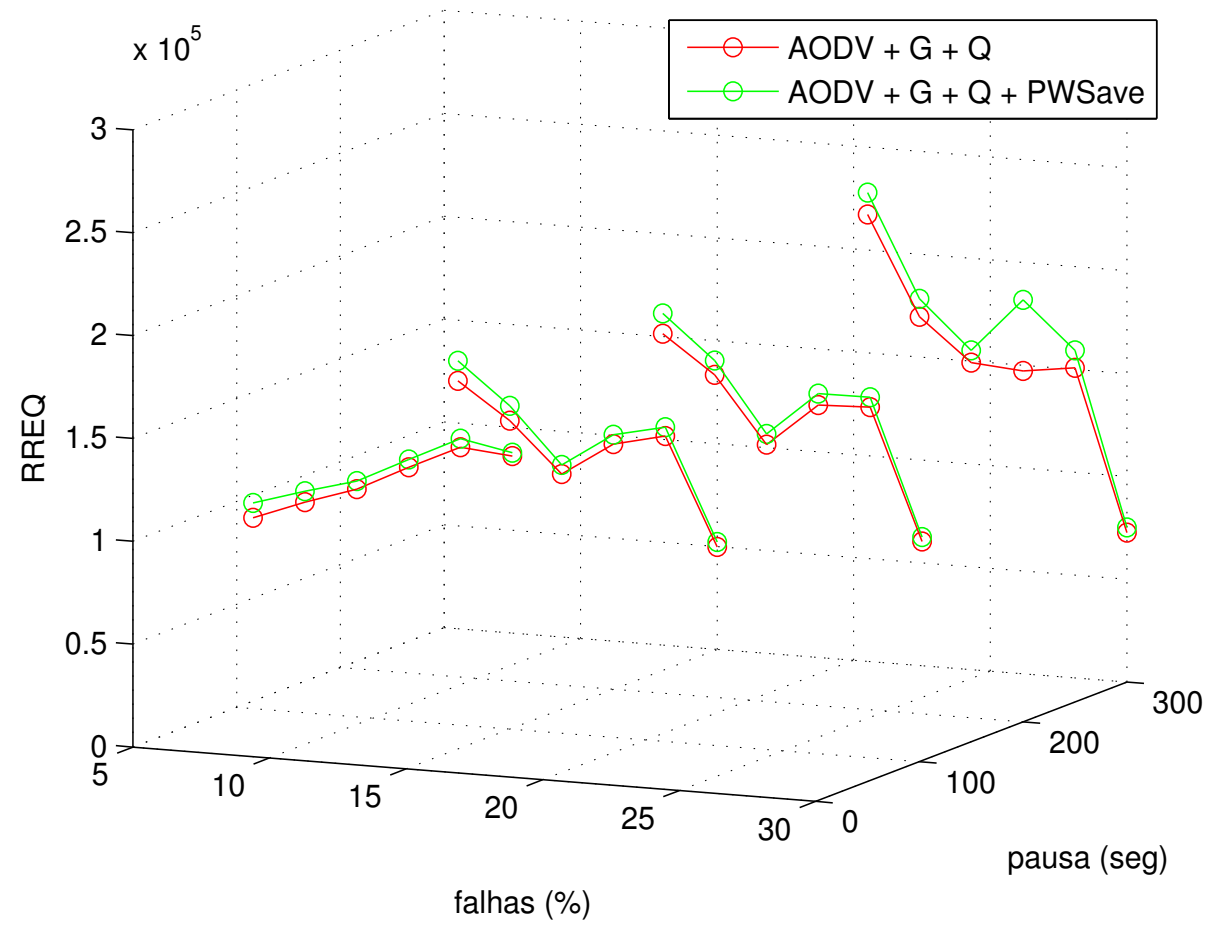

Figura 4.29: RREQ

Cada figura mostra as diferentes porcentagens de falha $(7,5,15,22,5$ e $30 \%)$.

A Figura 4.34 compara os resultados do consumo de energia dos cenários com Gossip e Quorum com e sem o algoritmo PWSave. Há uma diminuição de $10 \%$ de consumo de energia com a adição do PWSave, que é explicado pelo 


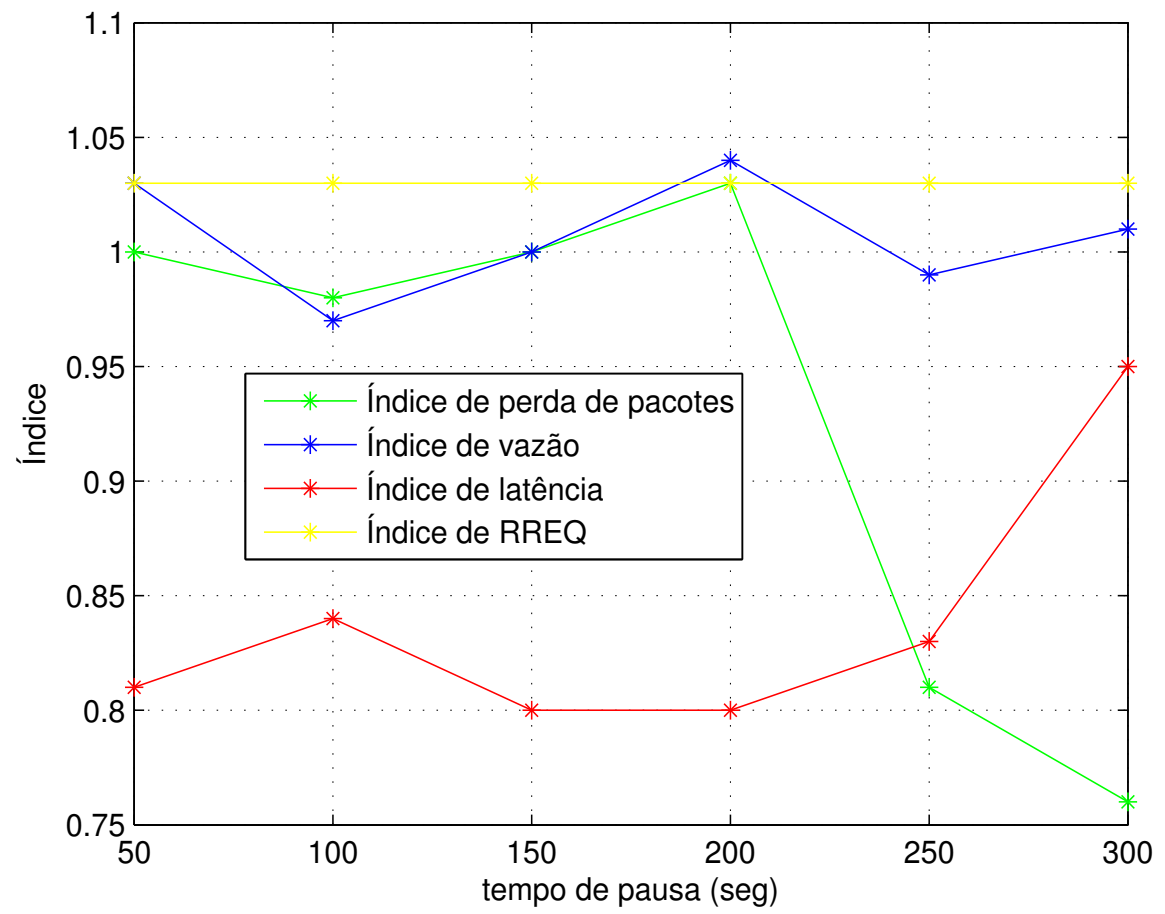

Figura 4.30: Índices de mérito para os parâmetros a 7,5\% de falha - Cenário B adormecimento dos nós. Constata-se que, caso seja necessário acordar os nós para troca de informações e/ou rotas, não há um aumento de energia superior à diminuição do consumo de energia causada pelo adormecimento dos nós. 


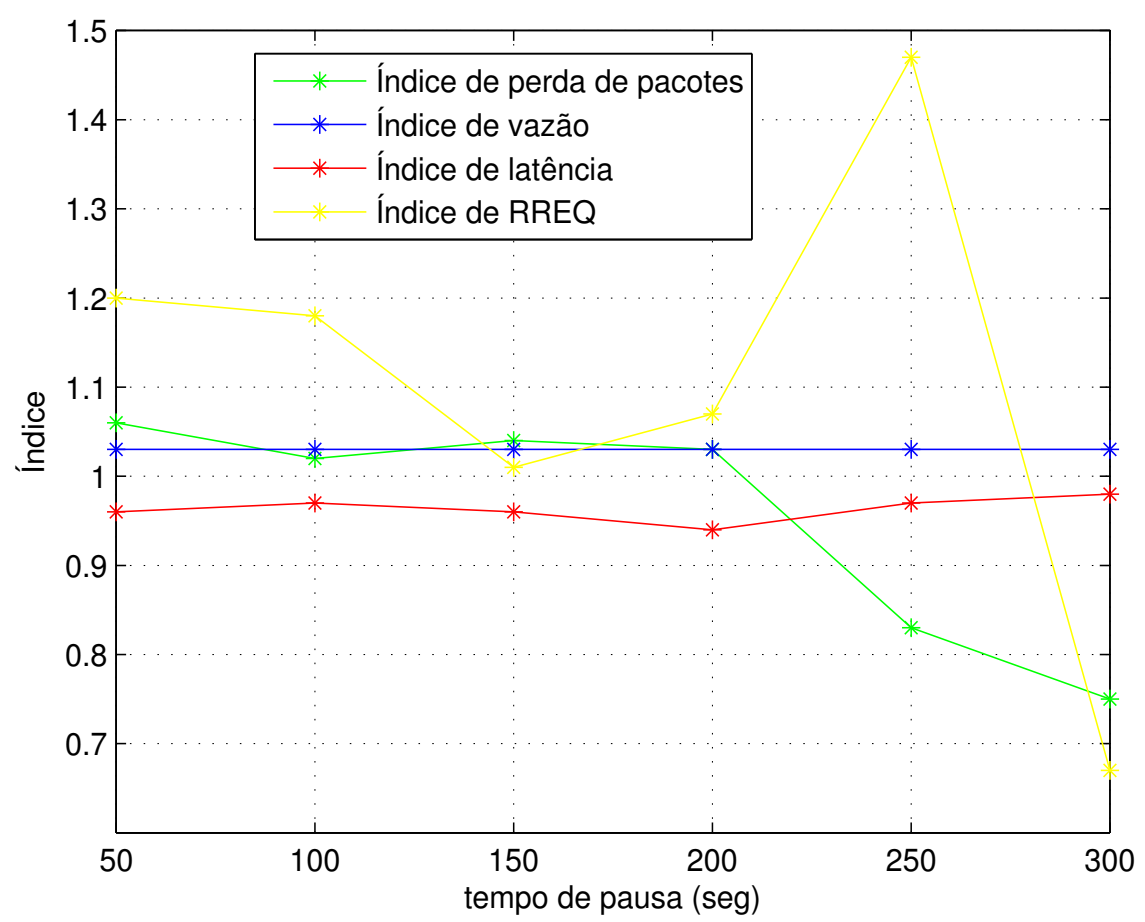

Figura 4.31: Índices de mérito de perda de pacotes a 15\% de falha - Cenário B

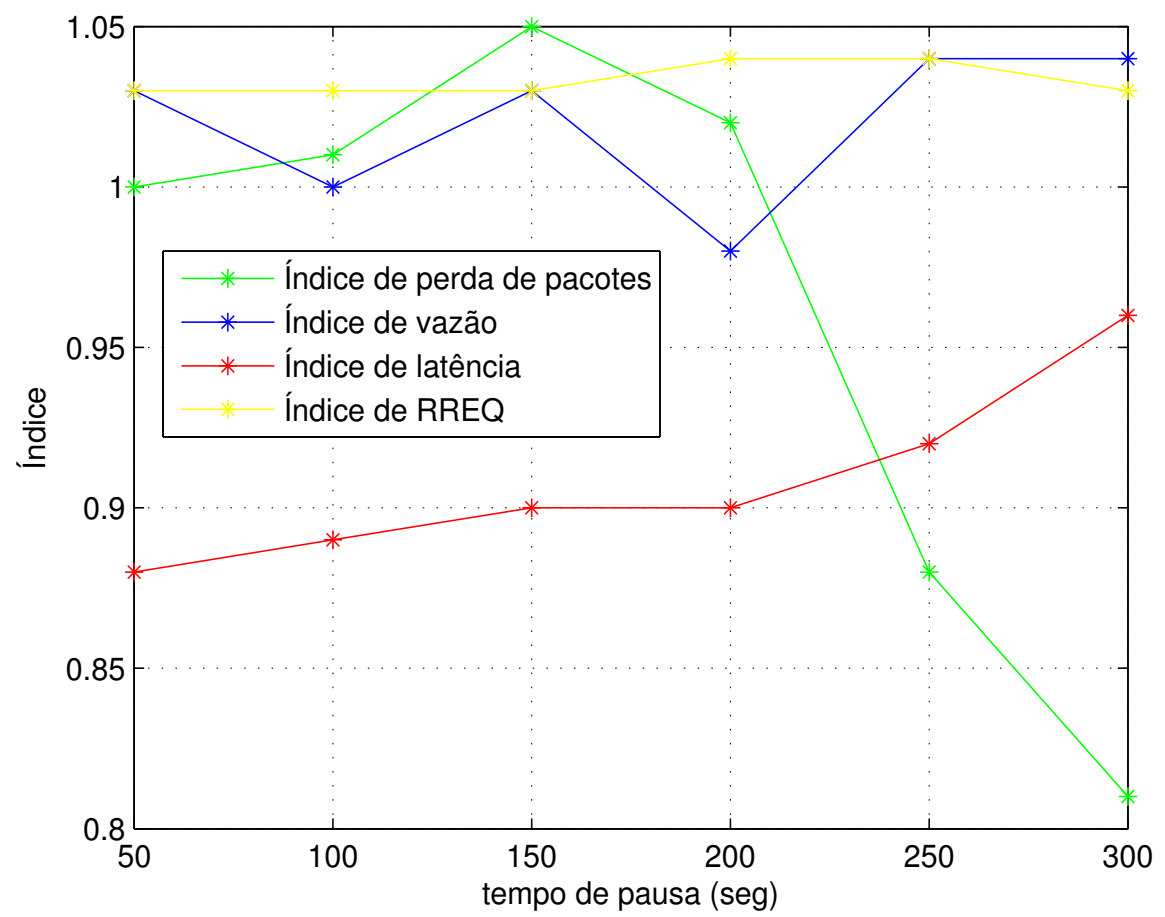

Figura 4.32: Índices de mérito de perda de pacotes a 22,5\% de falha - Cenário 


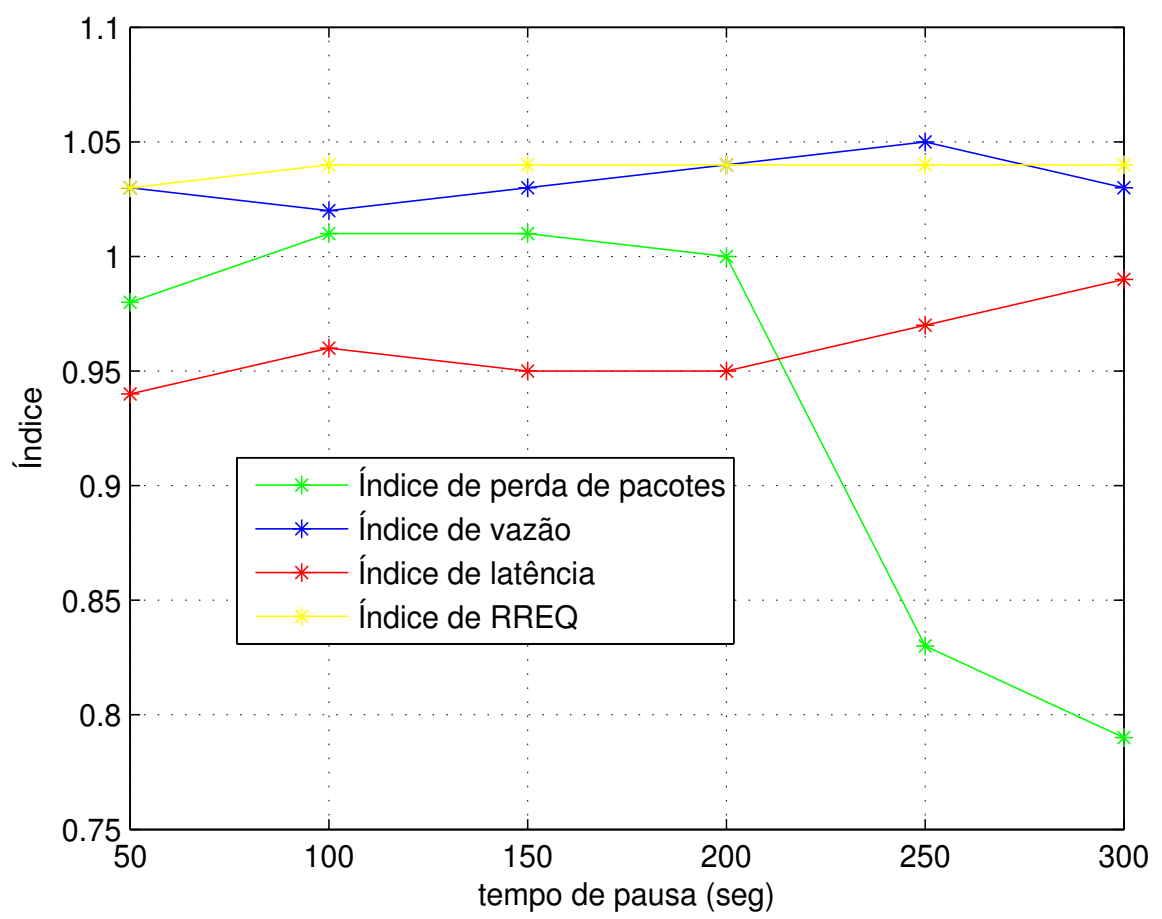

Figura 4.33: Índices de mérito de perda de pacotes a 30\% de falha - Cenário B

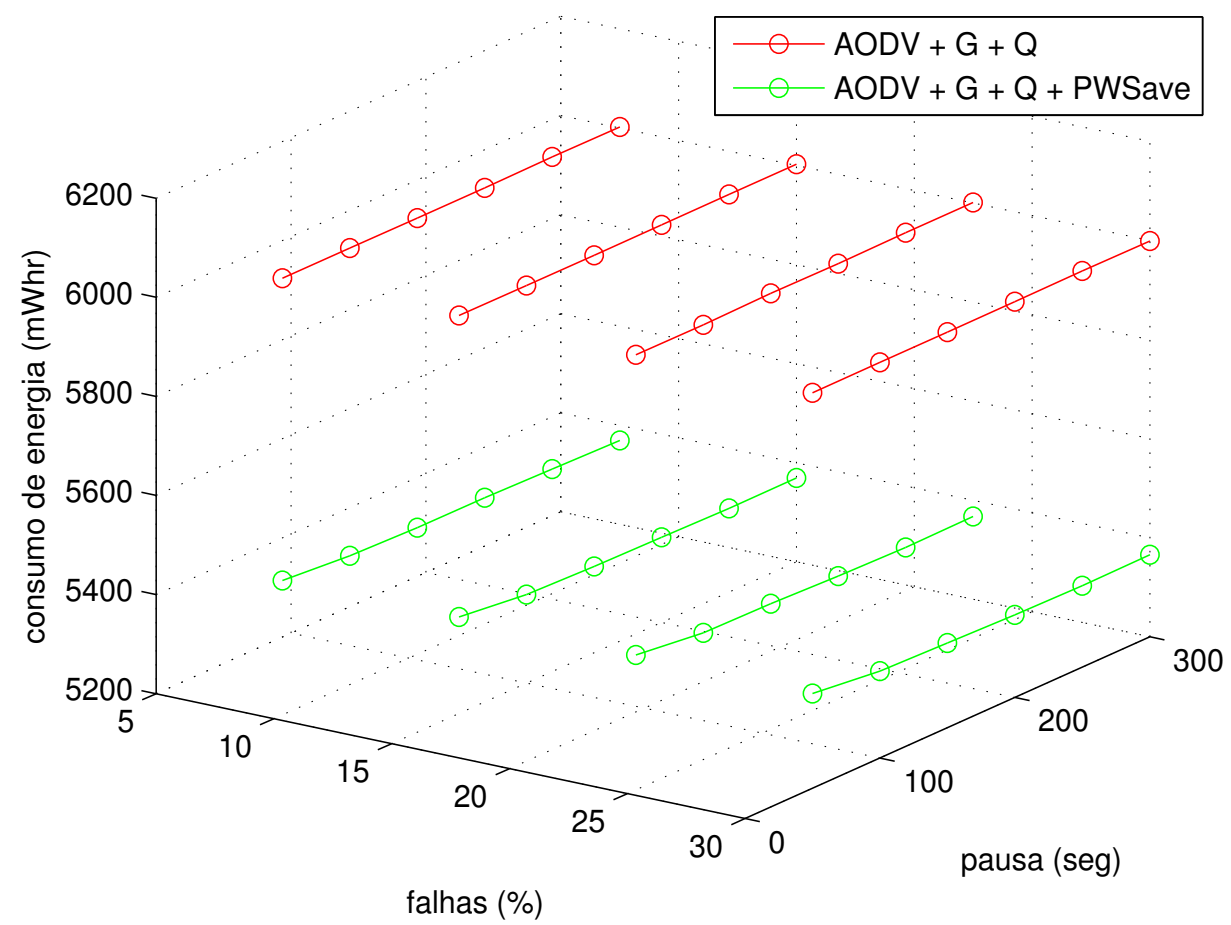

Figura 4.34: Consumo de Energia 


\section{Conclusão}

Neste capítulo são abordadas as principais contribuições deste trabalho, assim como as sugestões para trabalho futuro.

\subsection{Sumário e Contribuições}

Conforme o enunciado da seção 1.3, o objetivo principal deste trabalho é analisar, em um ambiente com e sem falhas, a associação do algoritmo probabilístico de disseminação de informação chamado Gossip e um sistema de Quorum com os protocolos de roteamento AODV e DSR, além de trabalhar características de economia de energia com a implementação do algoritmo PWSave associado ao Gossip e ao sistema de Quorum no protocolo AODV.

O desempenho da rede é avaliado pela medida de entrega de pacotes (\%), RREQ, vazão (bps), atraso (seg) e consumo de energia (mWhr).

Primeiramente, foram feitas simulações com o Gossip e com a análise dos resultados nota-se uma diminuição de 14\% no número de RREQ, no ambiente com tempo de pausa de 100 segundos com o protocolo AODV. Posteriormente, foi adicionado ao Gossip o sistema de Quorum que proporcionou menor perda de pacotes com uma diminuição de 20,19\% no cenário B, com aplicação de $15 \%$ de falhas em um ambiente com tempo de pausa de 100 segundos com o protocolo AODV. Também foi implementado, em paralelo com as soluções do algoritmo Gossip e do sistema de Quorum, o algoritmo PWSave, para proporcionar uma diminuição no consumo de energia nos nós da rede. Comparando-se a solução da associação do AODV + Gossip + Quorum com a solução da associação do AODV + Gossip + Quorum + PWSave obteve-se uma economia de energia de 10,39\% no cenário B, com aplicação de $15 \%$ de falhas em um ambiente com tempo de pausa de 100 segundos com o protocolo AODV.

Alguns trabalhos de economia de energia [38] demonstram uma perda de pacotes de $10 \%$ em cenários com $10 \%$ de probabilidade de falhas, mas não mostram 
outros parâmetros, como latência, e não explicitam a quantidade de energia total que foi economizada. Em [39] trabalha-se com uma taxa de consumo médio de energia de aproximadamente 32\%-33\% em um cenário sem falhas e em torno dos $12 \%$ em um cenário de falhas, que varia de $5 \%$ a 25\%. Em [39] são mostrados bons resultados na economia de energia e uma latência apenas um pouco sacrificada, como no caso da implementação do PWSave. Contudo, o esquema de adormecimento dos nós proposto em [39] é baseado em um agendamento em que os nós agendados para permanecer acordados não devem falhar, e isso, do ponto de vista real, pode ser um ponto negativo para uma rede.

Portanto, a solução de associação do Gossip + Quorum + PWSave no protocolo de roteamento AODV neste trabalho, mostra que há uma diminuição do consumo de energia sem afetar demais os outros parâmetros.

\subsection{Sugestões para Trabalhos Futuros}

Algumas extensões podem ser indicadas para esta dissertação. A seguir, são enumeradas algumas sugestões de trabalhos futuros:

1. Estudar e fazer uma análise mais detalhada em cenário de 100 segundos de tempo de pausa que possui características discrepantes em relação aos outros cenários de tempo de pausa;

2. Otimizar os parâmetros de adormecimento e despertar dos nós no algoritmo PWSave.

3. Trabalhar na implementação de características power saving do DSR;

4. Estudar outros protocolos de roteamento para comparação com o AODV e DSR. 


\section{Referências}

[1] LING, H.; MOSSE, D.; ZNATI, T. Coverage-based probabilistic forwarding in ad hoc routing. IEEE International Conference on Computer Communications and Networks, v. 4, n. 18, October 2005.

[2] LUO, J.; EUGSTER, P. T.; HUBAUX, J.-P. Pilot: Probabilistic lightweight group communication system for ad hoc networks. IEEE Symposium on Mobile Computing, v. 3, n. 2, April 2004.

[3] MURTHY, C. S. R.; GHOSAL, B. S. Ad Hoc Wireless Networks. [S.l.]: Prentice Hall, 2005.

[4] LENDERS, V.; WAGNER, J.; MAY, M. Measurements from 802.11b mobile ad hoc networks. IEEE International Symposium on a Mobile and Multimedia Netwoks, v. 4, n. 18, June 2006.

[5] SZU-CHI; KUO, S.-Y. Communication strategies for heartbeat-style failure detectors in wireless ad hoc networks. IEEE International Conference on Dependable Systems and Networks, v. 4, n. 18, June 2003.

[6] SINGH, S.; WOO, M.; RAGHAVENDRA, C. S. Power-aware routing in mobile ad hoc networks. Proceedings of the 4th annual ACM/IEEE International Conference on Mobile Computing and Networking, v. 4, n. 18, October 1998.

[7] CHANG, J.; TASSIULAS, L. Energy conserving routing in wireless ad-hoc network. Proceedings of the IEEE International Conference on Computer Communications, v. 9, n. 10, March 2000.

[8] LI, X.; ZI-WEN, W.; BAO-YU, Z. Tpbdsr: a new dsr-based energy saving routing in manet. International Conference on Computer Networks and Mobile Computing, v. 20, n. 13, October 2003.

[9] KIM; CHO. Enhanced power-saving mechanism for broadcast and multicast service in wlan. IEEE Communications Letters, v. 9, n. 6, June 2005.

[10] HUANG, W.-J.; CHIU, F.-H.; KUO, C. C. J. Comparison of power control schemes for relay sensor networks. Global Telecommunications Conference, v. 26, n. 30, November 2007.

[11] SPOHN, M. A.; GARCIA-LUNA-ACEVES, J. Multicasting in ad hoc networks in the context of multiple channels and multiple interfaces. IEEE/ACM Trans. on Networking, v. 06, n. 3, May 2005.

[12] SU, W. W.; LEE, S.-J. An adaptive and fault-tolerant gateway assignment in sensor networks. IEEE International Conference on Mobile Ad-Hoc and Sensor Systems, v. 06, n. 5, April 2004. 
[13] COSTA, P.; PICCO, G. P. Semi-probabilistic content-based publishsubscribe. IEEE International Conference on Mobile Ad-Hoc and Sensor Systems, v. 07, n. 10, May 2005.

[14] SHI, Z.; SRIMANI, P. K. An efficient distributed protocol for online gossiping problem. IEEE International Conference on Intelligent Agent Technology, v. 03, n. 8, May 2005.

[15] JIANG, T.; LI, Q. A self-stabilizing distributed multicast algorithm for mobile ad-hoc networks. IEEE International Conference on Computer and Information Technology, v. 30, n. 13, April 2004.

[16] CHOU, C.-H.; SSSU, K.-F.; JIAU, H. C. A distributed location service for reducing query failures in geographic ad hoc routing. IEEE International Conference on Computer and Information Technology, v. 15, n. 1, April 2005.

[17] MELAMED, R.; KEIDAR, I.; BAREL, Y. Octopus: A fault-tolerant and efficient ad-hoc routing protocol. IEEE Symposium on Reliable Distributed Systems, v. 20, n. 9, May 2005.

[18] WU, W.; CAO, J.; YANG, J. A scalable mutual exclusion algorithm for mobile ad hoc networks. IEEE/ACM Trans. on Networking, v. 3, n. 9, May 2005 .

[19] MASUM, S. M.; ALI, A. A. Asynchronous l-exclusion in mobile ad hoc networks. IEEE/ACM Trans. on Networking, v. 8, n. 4, June 2006.

[20] MUELLER, S.; GHOSAL, D. Analysis of a distributed algorithm to determine multiple routes with path diversity in ad hoc networks. IEEE Symposium on Mobile Computing, v. 4, n. 7, May 2005.

[21] MUELLER, S.; GHOSAL, D. Routing protocol based on fuzzy regression for manet. Proceedings of the Third International Conference on Machine Learning and Cybernetics, v. 4, n. 10, August 2004.

[22] AGARWAL, A.; JAIN, B. Routing reliability analysis of segmented backup paths in mobile ad hoc networks. IEEE International Conference on Mobile Ad-Hoc and Sensor Systems, v. 4, n. 10, April 2005.

[23] WANG, G. et al. A novel qos multicast model in mobile ad hoc networks. IEEE International Parallel and Distributed Processing Symposium, v. 3, n. 7, May 2005.

[24] LIU, B. H. et al. A self-organizing, location-aware media access control protocol for ds-cdma sensor networks. IEEE International Conference on Mobile Ad-Hoc and Sensor Systems, v. 4, n. 10, April 2004.

[25] IEEE/2001. IEEE 802.3 CSMA/CD (ETHERNET). Disponível em: $<$ http://www.ieee802.org/3/>.

[26] JUNK, S.; HUNDEWALE, N.; ZELIKOVSKY, A. Node caching enhancement of reactive ad hoc routing protocols [manet]. IEEE Wireless Communications and Networking Conference, v. 4, n. 13-17, March 2005. 
[27] MOGHIM, N.; HENDESSI, F.; MOVEHHEDINIA, N. An improvement on adhoc wireless network routing based on aodv. The 8th International Conference on Computer Communication Systems, v. 2, n. 11, October 2002.

[28] XIAOFENG, Z. et al. Stable enhancement for aodv routing protocol. 14th IEEE Proceedings on Personal, Indoor and Mobile Radio Communications, v. 1, n. 14, May 2003.

[29] JAIHYUNG, C.; BREEN, J. A flood routing method for data networks information, v. 3, n. 10, June 1997.

[30] JENKINS, K.; HOPKINSON, K.; BIRMAN, K. A gossip protocol for subgroup multicast. IEEE International Parallel and Distributed Processing Symposium, v. 3, n. 7, May 2005.

[31] ATANASIO, A.; LI, L. T. Algoritmos epidêmicos em computação distribuída. Disponível em: <http://www.ic.unicamp.br/atanasio/PUB/disciplinas/compDistrib/2007 UnicampAlgoritmosEpidemicos.pdf $>$.

[32] WOOL, A. Quorum systems in replicated databases. IEEE International Conference on Computer Communications and Networks, v. 7, n. 10, October 1998.

[33] WOESNER, H. Power-saving mechanisms in emerging standards for wireless lans: the mac level perspective. IEEE Personal Commun., v. 5, n. 5, June 1998.

[34] LAB, D.-E. S. IDES 2.1 - Integrated DiscreteEvent Systems Softwares Version 2.1. Disponível em: $<$ http://www.ece.queensu.ca/directory/faculty/Rudie.html >.

[35] NS-2. NS-2 Network Simulator. Disponível em: $<$ http://www.isi.edu/nsnam/ns/>.

[36] UCLA. GLOMOSIM - Global Mobile Information System Simulation Library. Disponível em: <http://pcl.cs.ucla.edu/projects/glomosim/>.

[37] MARGI, C. B.; OBRACZKA, K. Instrumenting networks simulators for evaluating energy consumption in power-aware ad-hoc network protocols. IEEE International Conference on Mobile Ad-Hoc and Sensor Systems, v. 4, n. 10, April 2006.

[38] GE, Y. et al. Green: A grid-based energy efficient probabilistic routing in wireless sensor networks. IEEE International Computation Symposium, v. 3, n. 7, May 2005.

[39] ZHOU, Z. et al. An energy-efficient data-dissemination protocol inwireless sensor networks. Proceedings of the 2006 International Symposium on a World of Wireless, Mobile and Multimedia Networks, v. 4, n. 3, June 2006. 


\section{Anexo A}

\section{Mapa Conceitual}

O mapa conceitual utilizado neste trabalho é ilustrado na página seguinte. 


\section{Anexo B - Tabelas referentes aos resultados de simulação}

As Tabelas 5.1 a 5.9 mostram os resultados referente à implementação de falhas nos cenários de simulação.

A Tabela 5.1 mostra os valores médios e desvios padrões da latência do cenário B.

A Tabela 5.2 mostra os valores médios e desvios padrões da vazão do cenário A e a Tabela 5.3 mostra os valores médios e desvios padrões da vazão do cenário B.

A Tabela 5.4 mostra os valores médios e desvios padrões para mensagens RREQ do cenário A e a Tabela 5.5 mostra os valores médios e desvios padrões para mensagens RREQ do cenário B.

A Tabela 5.6 mostra os valores médios e desvios padrões para o consumo de energia do cenário A e a Tabela 5.7 mostra os valores médios e desvios padrões para o consumo de energia do cenário B.

A Tabela 5.8 apresenta os valores médios e desvios padrões em relação à perda de pacotes nas falhas do cenário A e a Tabela 5.9 apresenta os valores médios e desvios padrões em relação à perda de pacotes nas falhas do cenário B.

As Tabelas 5.10 a 5.19 mostram os resultados referente à implementação do algoritmo PWSave.

A Tabela 5.10 compara os resultados da perda de pacotes do cenário de simulação com Gossip e Quorum, por simplificação nomeado de $\mathrm{AODV}+\mathrm{G}+\mathrm{Q}$, com e sem o algoritmo PWSave no cenário A de falha. A Tabela 5.11 faz a mesma comparação anterior, porém no cenário B.

A Tabela 5.12 compara os resultados da latência dos cenários com Gossip e Quorum com e sem o algoritmo PWSave no cenário A e a Tabela 5.13 compara os resultados da latência dos cenários com Gossip e Quorum com e sem o algoritmo PWSave no cenário B. 
A Tabela 5.14 compara os resultados da vazão dos cenários com Gossip e Quorum com e sem o algoritmo PWSave no cenário A e a Tabela 5.15 compara os resultados da vazão dos cenários com Gossip e Quorum com e sem o algoritmo PWSave no cenário B.

A Tabela 5.16 compara os resultados de RREQ dos cenários com Gossip e Quorum com e sem o algoritmo PWSave no cenário A e a Tabela 5.17 compara os resultados de RREQ dos cenários com Gossip e Quorum com e sem o algoritmo PWSave no cenário B.

A Tabela 5.18 compara os resultados do consumo de energia dos cenários com Gossip e Quorum com e sem o algoritmo PWSave no cenário A e a Tabela 5.19 no cenário B.

Tabela 5.1: Valores médios e desvios padrões para latência do Cenário B em segundos

\begin{tabular}{ccccc}
\hline Falhas $(\%)$ & $\begin{array}{c}\text { Tempo de } \\
\text { pausa }(\mathrm{s})\end{array}$ & AODV & AODV $+\mathrm{G}$ & AODV $+\mathrm{G}+\mathrm{Q}$ \\
\hline 7,5 & 0 & $2,47 \pm 0,04$ & $2,47 \pm 0,05$ & $2,48 \pm 0,04$ \\
7,5 & 50 & $2,47 \pm 0,04$ & $2,49 \pm 0,05$ & $2,49 \pm 0,04$ \\
7,5 & 100 & $2,46 \pm 0,05$ & $2,49 \pm 0,04$ & $2,49 \pm 0,04$ \\
7,5 & 150 & $2,47 \pm 0,06$ & $2,49 \pm 0,05$ & $2,47 \pm 0,05$ \\
7,5 & 200 & $2,47 \pm 0,04$ & $2,49 \pm 0,06$ & $2,47 \pm 0,05$ \\
7,5 & 250 & $2,45 \pm 0,04$ & $2,43 \pm 0,04$ & $2,45 \pm 0,05$ \\
15 & 0 & $2,84 \pm 0,05$ & $2,88 \pm 0,04$ & $2,88 \pm 0,04$ \\
15 & 50 & $2,92 \pm 0,04$ & $2,94 \pm 0,04$ & $2,95 \pm 0,05$ \\
15 & 100 & $2,89 \pm 0,05$ & $2,90 \pm 0,04$ & $2,93 \pm 0,06$ \\
15 & 150 & $2,91 \pm 0,04$ & $2,91 \pm 0,05$ & $2,97 \pm 0,04$ \\
15 & 200 & $2,89 \pm 0,04$ & $2,88 \pm 0,04$ & $2,94 \pm 0,05$ \\
15 & 250 & $2,86 \pm 0,05$ & $2,86 \pm 0,05$ & $2,87 \pm 0,06$ \\
22,5 & 0 & $3,22 \pm 0,04$ & $3,29 \pm 0,04$ & $3,33 \pm 0,05$ \\
22,5 & 50 & $3,31 \pm 0,06$ & $3,38 \pm 0,05$ & $3,39 \pm 0,05$ \\
22,5 & 100 & $3,31 \pm 0,05$ & $3,37 \pm 0,04$ & $3,39 \pm 0,05$ \\
22,5 & 150 & $3,29 \pm 0,04$ & $3,32 \pm 0,04$ & $3,42 \pm 0,04$ \\
22,5 & 200 & $3,20 \pm 0,05$ & $3,27 \pm 0,06$ & $3,30 \pm 0,04$ \\
22,5 & 250 & $3,24 \pm 0,04$ & $3,27 \pm 0,05$ & $3,36 \pm 0,05$ \\
30 & 0 & $3,76 \pm 0,06$ & $3,84 \pm 0,05$ & $3,95 \pm 0,04$ \\
30 & 50 & $3,78 \pm 0,05$ & $3,86 \pm 0,05$ & $3,95 \pm 0,05$ \\
30 & 100 & $3,79 \pm 0,04$ & $3,84 \pm 0,04$ & $3,94 \pm 0,07$ \\
30 & 150 & $3,77 \pm 0,04$ & $3,84 \pm 0,04$ & $3,94 \pm 0,04$ \\
30 & 200 & $3,79 \pm 0,05$ & $3,83 \pm 0,05$ & $3,94 \pm 0,05$ \\
30 & 250 & $3,78 \pm 0,04$ & $3,83 \pm 0,04$ & $3,96 \pm 0,06$ \\
\hline & & & &
\end{tabular}


Tabela 5.2: Valores médios e desvios padrões para vazão do Cenário A em $\mathrm{bits} / \mathrm{seg}$.

\begin{tabular}{ccccc}
\hline Falhas (\%) & $\begin{array}{c}\text { Tempo de } \\
\text { pausa }(\mathrm{s})\end{array}$ & AODV & AODV $+\mathrm{G}$ & AODV $+\mathrm{G}+\mathrm{Q}$ \\
\hline 7,5 & 0 & $197166,50 \pm 527,19$ & $193370,50 \pm 533,12$ & $197812,80 \pm 499,51$ \\
7,5 & 50 & $118560,10 \pm 613,01$ & $118047,90 \pm 498,34$ & $130128,40 \pm 501,38$ \\
7,5 & 100 & $118293,67 \pm 432,07$ & $117633,83 \pm 478,05$ & $130895,07 \pm 500,78$ \\
7,5 & 150 & $118293,67 \pm 499,18$ & $117778,90 \pm 501,23$ & $136998,60 \pm 598,16$ \\
7,5 & 200 & $128623,78 \pm 423,13$ & $122109,21 \pm 500,08$ & $193782,83 \pm 498,56$ \\
7,5 & 250 & $129624,55 \pm 409,17$ & $128335,62 \pm 456,70$ & $197903,41 \pm 500,45$ \\
15 & 0 & $207745,60 \pm 461,01$ & $207496,70 \pm 433,07$ & $218766,10 \pm 570,58$ \\
15 & 50 & $117553,30 \pm 415,60$ & $117079,80 \pm 409,88$ & $129364,60 \pm 545,33$ \\
15 & 100 & $128267,56 \pm 455,70$ & $137998,04 \pm 409,76$ & $149833,23 \pm 503,67$ \\
15 & 150 & $138168,70 \pm 501,67$ & $137883,00 \pm 514,39$ & $145348,10 \pm 564,37$ \\
15 & 200 & $152699,09 \pm 453,01$ & $151562,14 \pm 498,76$ & $162974,29 \pm 550,34$ \\
15 & 250 & $159547,56 \pm 502,32$ & $159047,39 \pm 498,22$ & $176587,79 \pm 498,06$ \\
22,5 & 0 & $109019,40 \pm 512,45$ & $108507,20 \pm 499,08$ & $178438,40 \pm 491,33$ \\
22,5 & 50 & $92313,20 \pm 500,78$ & $91774,94 \pm 497,05$ & $125201,15 \pm 525,85$ \\
22,5 & 100 & $93030,89 \pm 493,02$ & $97845,09 \pm 476,55$ & $130544,91 \pm 502,09$ \\
22,5 & 150 & $115427,60 \pm 402,65$ & $115061,14 \pm 517,12$ & $127861,37 \pm 559,75$ \\
22,5 & 200 & $139209,09 \pm 491,02$ & $138759,52 \pm 486,45$ & $146988,34 \pm 499,06$ \\
22,5 & 250 & $147137,33 \pm 511,24$ & $147014,03 \pm 503,77$ & $160982,18 \pm 510,56$ \\
30 & 0 & $119703,70 \pm 497,40$ & $119480,20 \pm 490,76$ & $132825,90 \pm 526,80$ \\
30 & 50 & $110096,90 \pm 502,98$ & $109262,56 \pm 469,50$ & $125972,51 \pm 508,07$ \\
30 & 100 & $110481,89 \pm 520,88$ & $116954,06 \pm 517,84$ & $128031,14 \pm 511,34$ \\
30 & 150 & $114435,40 \pm 493,34$ & $114117,20 \pm 480,67$ & $129395,40 \pm 526,67$ \\
30 & 200 & $112508,78 \pm 522,98$ & $112034,52 \pm 502,23$ & $127983,26 \pm 478,90$ \\
30 & 250 & $116324,89 \pm 499,70$ & $115928,02 \pm 487,56$ & $133049,37 \pm 498,67$ \\
\hline & & & &
\end{tabular}


Tabela 5.3: Valores médios e desvios padrões para vazão do Cenário B em $\mathrm{bits} / \mathrm{seg}$.

\begin{tabular}{ccccc}
\hline Falhas (\%) & $\begin{array}{c}\text { Tempo de } \\
\text { pausa }(\mathrm{s})\end{array}$ & AODV & AODV $+\mathrm{G}$ & AODV $+\mathrm{G}+\mathrm{Q}$ \\
\hline 7,5 & 0 & $197134,12 \pm 491,23$ & $193367,31 \pm 423,78$ & $198823,43 \pm 456,34$ \\
7,5 & 50 & $117512,33 \pm 501,23$ & $117022,08 \pm 512,89$ & $130117,36 \pm 544,12$ \\
7,5 & 100 & $117982,67 \pm 493,24$ & $117645,83 \pm 500,01$ & $136876,34 \pm 531,74$ \\
7,5 & 150 & $117287,32 \pm 511,24$ & $116767,94 \pm 502,34$ & $136976,12 \pm 543,16$ \\
7,5 & 200 & $128621,14 \pm 511,98$ & $122113,14 \pm 504,57$ & $164778,11 \pm 465,35$ \\
7,5 & 250 & $130645,50 \pm 470,98$ & $130325,30 \pm 492,56$ & $167941,98 \pm 512,45$ \\
15 & 0 & $207737,13 \pm 473,21$ & $207488,75 \pm 497,39$ & $218776,45 \pm 512,08$ \\
15 & 50 & $117567,24 \pm 491,23$ & $117075,28 \pm 507,88$ & $130359,13 \pm 516,96$ \\
15 & 100 & $128243,16 \pm 499,80$ & $129984,24 \pm 514,56$ & $149825,75 \pm 534,56$ \\
15 & 150 & $138159,14 \pm 486,45$ & $137876,24 \pm 503,22$ & $143352,45 \pm 535,11$ \\
15 & 200 & $152676,23 \pm 469,41$ & $151513,45 \pm 481,24$ & $162967,46 \pm 578,14$ \\
15 & 250 & $159546,12 \pm 492,03$ & $151034,34 \pm 507,53$ & $176576,87 \pm 512,87$ \\
22,5 & 0 & $129026,13 \pm 503,98$ & $118522,16 \pm 472,09$ & $178429,22 \pm 502,31$ \\
22,5 & 50 & $92346,11 \pm 511,24$ & $91769,90 \pm 508,67$ & $127215,35 \pm 566,09$ \\
22,5 & 100 & $93041,86 \pm 521,56$ & $99849,29 \pm 493,99$ & $131526,64 \pm 523,33$ \\
22,5 & 150 & $115437,12 \pm 482,44$ & $115058,31 \pm 501,55$ & $129858,25 \pm 522,65$ \\
22,5 & 200 & $139212,87 \pm 493,60$ & $138764,44 \pm 459,63$ & $146965,17 \pm 433,12$ \\
22,5 & 250 & $147143,35 \pm 491,93$ & $147021,35 \pm 510,98$ & $163976,65 \pm 521,57$ \\
30 & 0 & $119712,34 \pm 499,73$ & $119498,12 \pm 528,54$ & $128844,15 \pm 567,22$ \\
30 & 50 & $110081,77 \pm 524,04$ & $109264,09 \pm 507,55$ & $125969,01 \pm 512,37$ \\
30 & 100 & $115475,09 \pm 491,02$ & $116945,23 \pm 519,62$ & $128014,16 \pm 524,14$ \\
30 & 150 & $114421,19 \pm 506,01$ & $114109,33 \pm 503,88$ & $129387,66 \pm 536,97$ \\
30 & 200 & $112524,18 \pm 492,33$ & $112045,13 \pm 493,87$ & $127936,64 \pm 498,20$ \\
30 & 250 & $116374,29 \pm 507,55$ & $115914,55 \pm 483,21$ & $130087,07 \pm 476,90$ \\
\hline & & & &
\end{tabular}


Tabela 5.4: Valores médios e desvios padrões para RREQ do Cenário A

\begin{tabular}{ccccc}
\hline Falhas $(\%)$ & Tempo & AODV & AODV $+\mathrm{G}$ & AODV $+\mathrm{G}+\mathrm{Q}$ \\
\multicolumn{5}{c}{ de } \\
pausa $(\mathrm{s})$
\end{tabular}


Tabela 5.5: Valores médios e desvios padrões para RREQ do Cenário B

\begin{tabular}{|c|c|c|c|c|}
\hline Falhas (\%) & $\begin{array}{c}\text { Tempo } \\
\text { de } \\
\text { pausa (s) }\end{array}$ & AODV & $\mathrm{AODV}+\mathrm{G}$ & $\mathrm{AODV}+\mathrm{G}+\mathrm{Q}$ \\
\hline 7,5 & 0 & $93912,31 \pm 4129,70$ & $85376,34 \pm 4023,56$ & $85994,61 \pm 4323,08$ \\
\hline 7,5 & 50 & $152578,33 \pm 4289,60$ & $102723,18 \pm 4350,50$ & $104367,23 \pm 4754,12$ \\
\hline 7,5 & 100 & $110865,17 \pm 4663,29$ & $102011,16 \pm 4778,90$ & $102362,33 \pm 4951,01$ \\
\hline 7,5 & 150 & $95675,87 \pm 5015,45$ & $88823,98 \pm 5123,95$ & $98978,44 \pm 5503,12$ \\
\hline 7,5 & 200 & $100018,23 \pm 4349,40$ & $98222,67 \pm 4590,43$ & $99981,67 \pm 4911,01$ \\
\hline 7,5 & 250 & $111912,13 \pm 4870,67$ & $99378,74 \pm 4070,56$ & $99994,80 \pm 4550,12$ \\
\hline 15 & 0 & $67065,28 \pm 5004,67$ & $48549,11 \pm 5011,23$ & $49827,75 \pm 5212,15$ \\
\hline 15 & 50 & $231293,11 \pm 4773,86$ & $176856,37 \pm 4990,65$ & $178855,03 \pm 4529,13$ \\
\hline 15 & 100 & $157900,49 \pm 4288,40$ & $149593,61 \pm 4743,98$ & $149938,12 \pm 4387,11$ \\
\hline 15 & 150 & $162826,30 \pm 4756,98$ & $113055,87 \pm 4802,36$ & $114322,09 \pm 4970,04$ \\
\hline 15 & 200 & $136981,39 \pm 4176,80$ & $120788,22 \pm 4033,67$ & $119186,73 \pm 4171,62$ \\
\hline 15 & 250 & $132699,12 \pm 4587,65$ & $113141,17 \pm 4312,98$ & $113431,86 \pm 4711,54$ \\
\hline 22,5 & 0 & $78099,03 \pm 5002,56$ & $59415,76 \pm 5054,87$ & $60358,13 \pm 5156,34$ \\
\hline 22,5 & 50 & $284461,77 \pm 5011,76$ & $205933,19 \pm 5007,34$ & $209734,78 \pm 5256,65$ \\
\hline 22,5 & 100 & $198122,34 \pm 4589,90$ & $179347,66 \pm 4311,98$ & $180033,91 \pm 4265,23$ \\
\hline 22,5 & 150 & $207344,35 \pm 5015,87$ & $135569,37 \pm 4655,86$ & $136489,33 \pm 4734,12$ \\
\hline 22,5 & 200 & $163827,87 \pm 4886,67$ & $145577,99 \pm 5000,87$ & $146044,66 \pm 4133,09$ \\
\hline 22,5 & 250 & $155333,54 \pm 4711,75$ & $134865,68 \pm 4529,87$ & $135397,02 \pm 4426,74$ \\
\hline 30 & 0 & $95223,07 \pm 4387,57$ & $72688,26 \pm 4550,67$ & $72634,99 \pm 4843,12$ \\
\hline 30 & 50 & $368771,22 \pm 4765,78$ & $274387,87 \pm 5003,67$ & $275634,05 \pm 4555,02$ \\
\hline 30 & 100 & $229658,33 \pm 4663,41$ & $215869,05 \pm 4707,56$ & $216286,67 \pm 5933,16$ \\
\hline 30 & 150 & $215933,60 \pm 5000,56$ & $181022,86 \pm 5002,65$ & $184269,99 \pm 5112,08$ \\
\hline 30 & 200 & $190036,44 \pm 4688,90$ & $169386,88 \pm 4712,65$ & $170556,04 \pm 7644,12$ \\
\hline 30 & 250 & $162714,05 \pm 7021,81$ & $161966,88 \pm 7656,90$ & $162464,04 \pm 8509,44$ \\
\hline
\end{tabular}


Tabela 5.6: Valores médios e desvios padrões para o consumo de energia do Cenário A em mWhr

\begin{tabular}{ccccc}
\hline Falhas $(\%)$ & $\begin{array}{c}\text { Tempo de } \\
\text { pausa }(\mathrm{s})\end{array}$ & AODV & AODV $+\mathrm{G}$ & AODV $+\mathrm{G}+\mathrm{Q}$ \\
\hline 7,5 & 0 & $6004,69 \pm 0,40$ & $6003,82 \pm 0,49$ & $6003,99 \pm 0,49$ \\
7,5 & 50 & $6006,00 \pm 0,40$ & $6004,31 \pm 0,50$ & $6004,52 \pm 0,50$ \\
7,5 & 100 & $6005,33 \pm 0,47$ & $6003,15 \pm 0,47$ & $6003,26 \pm 0,47$ \\
7,5 & 150 & $6003,74 \pm 0,40$ & $6002,15 \pm 0,49$ & $6003,12 \pm 0,49$ \\
7,5 & 200 & $6003,92 \pm 0,45$ & $6002,32 \pm 0,49$ & $6002,54 \pm 0,49$ \\
7,5 & 250 & $6003,31 \pm 0,49$ & $6002,23 \pm 0,43$ & $6003,77 \pm 0,43$ \\
15 & 0 & $6004,91 \pm 0,40$ & $6004,33 \pm 0,47$ & $6004,40 \pm 0,47$ \\
15 & 50 & $6006,33 \pm 0,40$ & $6004,83 \pm 0,40$ & $6004,63 \pm 0,49$ \\
15 & 100 & $6004,59 \pm 0,50$ & $6002,76 \pm 0,40$ & $6003,25 \pm 0,44$ \\
15 & 150 & $6003,86 \pm 0,40$ & $6002,89 \pm 0,45$ & $6003,14 \pm 0,53$ \\
15 & 200 & $6004,12 \pm 0,40$ & $6002,42 \pm 0,40$ & $6003,67 \pm 0,44$ \\
15 & 250 & $6003,13 \pm 0,47$ & $6002,43 \pm 0,45$ & $6003,98 \pm 0,45$ \\
22,5 & 0 & $6001,59 \pm 0,45$ & $6000,46 \pm 0,40$ & $6000,87 \pm 0,51$ \\
22,5 & 50 & $6001,30 \pm 0,40$ & $5999,61 \pm 0,46$ & $6000,51 \pm 0,50$ \\
22,5 & 100 & $6000,87 \pm 0,49$ & $5998,32 \pm 0,48$ & $5999,67 \pm 0,47$ \\
22,5 & 150 & $6000,59 \pm 0,47$ & $6000,59 \pm 0,40$ & $6000,00 \pm 0,43$ \\
22,5 & 200 & $6000,76 \pm 0,40$ & $5999,98 \pm 0,40$ & $5999,66 \pm 0,50$ \\
22,5 & 250 & $6003,18 \pm 0,40$ & $6000,46 \pm 0,40$ & $6001,04 \pm 0,42$ \\
30 & 0 & $6000,63 \pm 0,50$ & $5999,63 \pm 0,50$ & $5998,70 \pm 0,40$ \\
30 & 50 & $5999,57 \pm 0,40$ & $5998,72 \pm 0,50$ & $5998,54 \pm 0,41$ \\
30 & 100 & $6000,84 \pm 0,46$ & $5999,12 \pm 0,46$ & $5998,59 \pm 0,48$ \\
30 & 150 & $5999,49 \pm 0,45$ & $5998,79 \pm 0,45$ & $5998,62 \pm 0,47$ \\
30 & 200 & $6000,94 \pm 0,43$ & $5999,14 \pm 0,45$ & $5998,80 \pm 0,48$ \\
30 & 250 & $6001,41 \pm 0,45$ & $5999,92 \pm 0,43$ & $5999,56 \pm 0,40$ \\
\hline & & & &
\end{tabular}


Tabela 5.7: Valores médios e desvios padrões para o consumo de energia do Cenário B em mWhr

\begin{tabular}{ccccc}
\hline Falhas $(\%)$ & $\begin{array}{c}\text { Tempo de } \\
\text { pausa }(\mathrm{s})\end{array}$ & AODV & AODV $+\mathrm{G}$ & AODV $+\mathrm{G}+\mathrm{Q}$ \\
\hline 7,5 & 0 & $6003,69 \pm 0,47$ & $6000,82 \pm 0,47$ & $6001,99 \pm 0,50$ \\
7,5 & 50 & $6002,00 \pm 0,50$ & $6001,31 \pm 0,50$ & $6002,72 \pm 0,50$ \\
7,5 & 100 & $6001,46 \pm 0,50$ & $6000,33 \pm 0,50$ & $6002,16 \pm 0,47$ \\
7,5 & 150 & $6001,74 \pm 0,47$ & $5999,76 \pm 0,46$ & $6001,52 \pm 0,49$ \\
7,5 & 200 & $6002,92 \pm 0,46$ & $5999,41 \pm 0,46$ & $6001,54 \pm 0,47$ \\
7,5 & 250 & $6003,31 \pm 0,45$ & $5999,78 \pm 0,45$ & $6002,77 \pm 0,43$ \\
15 & 0 & $6002,91 \pm 0,46$ & $6002,33 \pm 0,47$ & $6002,60 \pm 0,47$ \\
15 & 50 & $6003,33 \pm 0,45$ & $6002,43 \pm 0,46$ & $6002,83 \pm 0,49$ \\
15 & 100 & $6003,12 \pm 0,45$ & $6002,76 \pm 0,45$ & $6002,25 \pm 0,46$ \\
15 & 150 & $6002,94 \pm 0,46$ & $6002,86 \pm 0,47$ & $6002,14 \pm 0,50$ \\
15 & 200 & $6002,87 \pm 0,47$ & $6002,42 \pm 0,45$ & $6002,47 \pm 0,45$ \\
15 & 250 & $6002,43 \pm 0,45$ & $6002,13 \pm 0,47$ & $6002,93 \pm 0,46$ \\
22,5 & 0 & $6001,59 \pm 0,45$ & $6000,46 \pm 0,45$ & $6000,87 \pm 0,51$ \\
22,5 & 50 & $6001,32 \pm 0,47$ & $5999,63 \pm 0,45$ & $5999,56 \pm 0,50$ \\
22,5 & 100 & $6000,86 \pm 0,50$ & $5998,98 \pm 0,45$ & $5998,67 \pm 0,47$ \\
22,5 & 150 & $6000,54 \pm 0,47$ & $6000,29 \pm 0,46$ & $6000,97 \pm 0,44$ \\
22,5 & 200 & $6000,74 \pm 0,50$ & $5999,95 \pm 0,47$ & $5999,66 \pm 0,50$ \\
22,5 & 250 & $6001,16 \pm 0,45$ & $6000,46 \pm 0,45$ & $6001,04 \pm 0,44$ \\
30 & 0 & $6000,67 \pm 0,47$ & $5999,63 \pm 0,47$ & $5998,87 \pm 0,43$ \\
30 & 50 & $5999,54 \pm 0,46$ & $5998,72 \pm 0,46$ & $5998,44 \pm 0,43$ \\
30 & 100 & $5999,84 \pm 0,46$ & $5999,12 \pm 0,46$ & $5998,59 \pm 0,45$ \\
30 & 150 & $5999,49 \pm 0,50$ & $5998,79 \pm 0,46$ & $5998,62 \pm 0,45$ \\
30 & 200 & $5999,94 \pm 0,46$ & $5999,14 \pm 0,46$ & $5998,80 \pm 0,45$ \\
30 & 250 & $6001,41 \pm 0,47$ & $6000,92 \pm 0,47$ & $6000,56 \pm 0,42$ \\
\hline & & & &
\end{tabular}


Tabela 5.8: Valores médios e desvios padrões para a taxa de perda de pacotes do Cenário A em \%

\begin{tabular}{|c|c|c|c|c|}
\hline Falhas (\%) & $\begin{array}{l}\text { Tempo de } \\
\text { pausa (s) }\end{array}$ & AODV & $\mathrm{AODV}+\mathrm{G}$ & $\mathrm{AODV}+\mathrm{G}+\mathrm{Q}$ \\
\hline 7,5 & 0 & $6,85 \pm 2,01$ & $13,40 \pm 2,11$ & $6,27 \pm 2,79$ \\
\hline 7,5 & 50 & $39,94 \pm 1,13$ & $41,56 \pm 1,01$ & $30,79 \pm 1,07$ \\
\hline 7,5 & 100 & $43,67 \pm 1,09$ & $41,17 \pm 1,23$ & $29,03 \pm 1,25$ \\
\hline 7,5 & 150 & $38,63 \pm 1,07$ & $41,45 \pm 1,02$ & $27,05 \pm 1,16$ \\
\hline 7,5 & 200 & $40,31 \pm 1,19$ & $41,15 \pm 1,32$ & $21,09 \pm 1,67$ \\
\hline 7,5 & 250 & $14,82 \pm 2,01$ & $15,41 \pm 2,13$ & $8,67 \pm 2,34$ \\
\hline 15 & 0 & $9,10 \pm 1,03$ & $17,05 \pm 1,09$ & $6,71 \pm 1,03$ \\
\hline 15 & 50 & $46,85 \pm 3,06$ & $47,13 \pm 3,11$ & $36,36 \pm 4,31$ \\
\hline 15 & 100 & $44,68 \pm 1,02$ & $42,54 \pm 1,06$ & $26,86 \pm 1,20$ \\
\hline 15 & 150 & $41,38 \pm 1,23$ & $43,07 \pm 1,34$ & $34,73 \pm 1,80$ \\
\hline 15 & 200 & $39,17 \pm 0,97$ & $41,04 \pm 0,99$ & $24,77 \pm 0,95$ \\
\hline 15 & 250 & $11,03 \pm 2,31$ & $15,38 \pm 2,11$ & $10,78 \pm 2,43$ \\
\hline 22,5 & 0 & $11,18 \pm 0,90$ & $16,36 \pm 0,87$ & $9,21 \pm 0,91$ \\
\hline 22,5 & 50 & $45,32 \pm 1,11$ & $47,93 \pm 1,03$ & $34,74 \pm 1,12$ \\
\hline 22,5 & 100 & $49,63 \pm 1,09$ & $47,39 \pm 1,13$ & $36,02 \pm 1,29$ \\
\hline 22,5 & 150 & $37,59 \pm 0,88$ & $40,65 \pm 0,87$ & $31,78 \pm 0,86$ \\
\hline 22,5 & 200 & $36,09 \pm 0,90$ & $39,42 \pm 0,91$ & $26,09 \pm 0,93$ \\
\hline 22,5 & 250 & $15,63 \pm 1,02$ & $16,12 \pm 1,12$ & $14,57 \pm 1,22$ \\
\hline 30 & 0 & $11,78 \pm 0,99$ & $16,58 \pm 0,89$ & $9,29 \pm 0,94$ \\
\hline 30 & 50 & $45,45 \pm 1,13$ & $48,10 \pm 1,01$ & $34,96 \pm 1,11$ \\
\hline 30 & 100 & $52,39 \pm 0,72$ & $49,83 \pm 0,78$ & $38,18 \pm 0,68$ \\
\hline 30 & 150 & $37,78 \pm 0,93$ & $41,84 \pm 0,97$ & $28,87 \pm 0,95$ \\
\hline 30 & 200 & $35,29 \pm 0,65$ & $39,68 \pm 0,71$ & $26,97 \pm 0,58$ \\
\hline 30 & 250 & $15,39 \pm 0,97$ & $18,87 \pm 0,93$ & $14,34 \pm 0,98$ \\
\hline
\end{tabular}


Tabela 5.9: Valores médios e desvios padrões para a taxa de perda de pacotes do Cenário B em \%

\begin{tabular}{|c|c|c|c|c|}
\hline Falhas (\%) & $\begin{array}{l}\text { Tempo de } \\
\text { pausa }(\mathrm{s})\end{array}$ & AODV & $\mathrm{AODV}+\mathrm{G}$ & $\mathrm{AODV}+\mathrm{G}+\mathrm{Q}$ \\
\hline 7,5 & 0 & $6,67 \pm 2,13$ & $13,32 \pm 2,11$ & $6,15 \pm 2,34$ \\
\hline 7,5 & 50 & $39,65 \pm 1,32$ & $41,35 \pm 1,09$ & $26,22 \pm 1,23$ \\
\hline 7,5 & 100 & $41,87 \pm 1,03$ & $43,33 \pm 0,98$ & $29,23 \pm 1,01$ \\
\hline 7,5 & 150 & $38,41 \pm 1,24$ & $40,98 \pm 1,12$ & $21,54 \pm 1,34$ \\
\hline 7,5 & 200 & $40,23 \pm 1,07$ & $42,39 \pm 1,03$ & $24,54 \pm 1,13$ \\
\hline 7,5 & 250 & $8,45 \pm 1,97$ & $15,41 \pm 2,01$ & $8,89 \pm 2,09$ \\
\hline 15 & 0 & $9,45 \pm 1,21$ & $17,48 \pm 1,51$ & $6,21 \pm 1,54$ \\
\hline 15 & 50 & $46,68 \pm 2,34$ & $47,38 \pm 3,76$ & $36,98 \pm 4,11$ \\
\hline 15 & 100 & $43,54 \pm 1,00$ & $44,84 \pm 1,03$ & $34,14 \pm 1,49$ \\
\hline 15 & 150 & $41,18 \pm 1,07$ & $43,51 \pm 1,12$ & $26,87 \pm 1,33$ \\
\hline 15 & 200 & $39,34 \pm 1,09$ & $41,45 \pm 1,04$ & $24,56 \pm 1,23$ \\
\hline 15 & 250 & $11,43 \pm 1,15$ & $15,78 \pm 1,12$ & $10,76 \pm 1,54$ \\
\hline 22,5 & 0 & $11,24 \pm 1,19$ & $16,67 \pm 1,21$ & $9,44 \pm 1,41$ \\
\hline 22,5 & 50 & $45,76 \pm 1,12$ & $47,42 \pm 1,33$ & $34,46 \pm 1,45$ \\
\hline 22,5 & 100 & $47,54 \pm 1,34$ & $49,49 \pm 1,56$ & $36,32 \pm 1,76$ \\
\hline 22,5 & 150 & $37,19 \pm 1,01$ & $40,53 \pm 0,99$ & $27,23 \pm 0,98$ \\
\hline 22,5 & 200 & $36,24 \pm 0,98$ & $39,57 \pm 1,02$ & $26,12 \pm 1,01$ \\
\hline 22,5 & 250 & $15,37 \pm 0,78$ & $19,65 \pm 0,78$ & $14,87 \pm 0,65$ \\
\hline 30 & 0 & $11,66 \pm 1,02$ & $16,87 \pm 1,01$ & $9,54 \pm 0,99$ \\
\hline 30 & 50 & $45,76 \pm 0,67$ & $48,88 \pm 0,65$ & $34,12 \pm 0,61$ \\
\hline 30 & 100 & $49,54 \pm 0,91$ & $52,22 \pm 0,83$ & $38,65 \pm 0,87$ \\
\hline 30 & 150 & $37,48 \pm 0,81$ & $41,65 \pm 0,78$ & $28,43 \pm 0,75$ \\
\hline 30 & 200 & $35,54 \pm 1,01$ & $39,65 \pm 0,93$ & $26,33 \pm 0,98$ \\
\hline 30 & 250 & $15,64 \pm 0,98$ & $18,32 \pm 1,00$ & $14,53 \pm 1,04$ \\
\hline
\end{tabular}


Tabela 5.10: Valores médios e desvios padrões para a taxa de perda de pacotes do Cenário A em \%

\begin{tabular}{cccc}
\hline Falhas $(\%)$ & $\begin{array}{c}\text { Tempo de } \\
\text { pausa }(\mathrm{s})\end{array}$ & AODV $+\mathrm{G}+\mathrm{Q}$ & AODV $+\mathrm{G}+\mathrm{Q}+\mathrm{PWSave}$ \\
\hline 7,5 & 0 & $6,27 \pm 2,79$ & $15,16 \pm 1,1$ \\
7,5 & 50 & $30,79 \pm 1,07$ & $29,93 \pm 1,03$ \\
7,5 & 100 & $29,03 \pm 1,25$ & $33,67 \pm 1,34$ \\
7,5 & 150 & $27,05 \pm 1,16$ & $28,33 \pm 1,07$ \\
7,5 & 200 & $21,09 \pm 1,67$ & $24,17 \pm 0,98$ \\
7,5 & 250 & $8,67 \pm 2,34$ & $17,10 \pm 2,03$ \\
15 & 0 & $6,71 \pm 1,03$ & $16,74 \pm 1,93$ \\
15 & 50 & $36,36 \pm 4,31$ & $35,78 \pm 2,09$ \\
15 & 100 & $26,86 \pm 1,20$ & $31,01 \pm 1,22$ \\
15 & 150 & $34,73 \pm 1,80$ & $30,56 \pm 0,93$ \\
15 & 200 & $24,77 \pm 0,95$ & $26,13 \pm 1,00$ \\
15 & 250 & $10,78 \pm 2,43$ & $19,22 \pm 1,31$ \\
22,5 & 0 & $9,21 \pm 0,91$ & $18,02 \pm 2,42$ \\
22,5 & 50 & $34,74 \pm 1,12$ & $38,43 \pm 0,97$ \\
22,5 & 100 & $36,02 \pm 1,29$ & $39,65 \pm 0,78$ \\
22,5 & 150 & $31,78 \pm 0,86$ & $34,15 \pm 0,95$ \\
22,5 & 200 & $26,09 \pm 0,93$ & $27,98 \pm 0,78$ \\
22,5 & 250 & $14,57 \pm 1,22$ & $22,13 \pm 1,14$ \\
30 & 0 & $9,29 \pm 0,94$ & $19,78 \pm 2,03$ \\
30 & 50 & $34,96 \pm 1,11$ & $40,55 \pm 1,98$ \\
30 & 100 & $38,18 \pm 0,68$ & $42,04 \pm 0,78$ \\
30 & 150 & $28,87 \pm 0,95$ & $34,79 \pm 1,01$ \\
30 & 200 & $26,97 \pm 0,58$ & $30,15 \pm 0,91$ \\
30 & 250 & $14,34 \pm 0,98$ & $25,67 \pm 1,49$ \\
\hline & & & \\
\hline
\end{tabular}


Tabela 5.11: Valores médios e desvios padrões para a taxa de perda de pacotes do Cenário B em \%

\begin{tabular}{cccc}
\hline Falhas $(\%)$ & $\begin{array}{c}\text { Tempo de } \\
\text { pausa }(\mathrm{s})\end{array}$ & AODV $+\mathrm{G}+\mathrm{Q}$ & AODV $+\mathrm{G}+\mathrm{Q}+\mathrm{PWSave}$ \\
\hline 7,5 & 0 & $6,15 \pm 2,34$ & $14,129 \pm 1,2$ \\
7,5 & 50 & $26,22 \pm 1,23$ & $30,12 \pm 1,54$ \\
7,5 & 100 & $29,33 \pm 1,01$ & $32,93 \pm 1,0$ \\
7,5 & 150 & $21,54 \pm 1,34$ & $29,65 \pm 0,87$ \\
7,5 & 200 & $24,54 \pm 1,13$ & $24,54 \pm 0,99$ \\
7,5 & 250 & $8,89 \pm 2,09$ & $17,87 \pm 1,11$ \\
15 & 0 & $6,21 \pm 1,54$ & $15,74 \pm 1,32$ \\
15 & 50 & $36,98 \pm 4,11$ & $35,98 \pm 1,43$ \\
15 & 100 & $34,14 \pm 1,49$ & $38,03 \pm 1,09$ \\
15 & 150 & $26,87 \pm 1,33$ & $30,87 \pm 0,87$ \\
15 & 200 & $24,56 \pm 1,23$ & $26,45 \pm 1,04$ \\
15 & 250 & $10,76 \pm 1,54$ & $19,82 \pm 1,15$ \\
22,5 & 0 & $9,44 \pm 1,41$ & $18,15 \pm 1,35$ \\
22,5 & 50 & $34,46 \pm 1,45$ & $38,67 \pm 0,99$ \\
22,5 & 100 & $36,32 \pm 1,76$ & $39,88 \pm 0,78$ \\
22,5 & 150 & $27,23 \pm 0,98$ & $32,68 \pm 0,98$ \\
22,5 & 200 & $26,12 \pm 1,01$ & $27,19 \pm 0,76$ \\
22,5 & 250 & $14,87 \pm 0,65$ & $21,78 \pm 1,32$ \\
30 & 0 & $9,54 \pm 0,99$ & $19,12 \pm 2,11$ \\
30 & 50 & $34,12 \pm 0,61$ & $40,02 \pm 1,54$ \\
30 & 100 & $38,65 \pm 0,87$ & $42,54 \pm 0,97$ \\
30 & 150 & $28,43 \pm 0,75$ & $34,13 \pm 1,05$ \\
30 & 200 & $26,33 \pm 0,98$ & $30,56 \pm 0,98$ \\
30 & 250 & $14,53 \pm 1,04$ & $25,01 \pm 1,06$ \\
\hline & & & \\
\hline
\end{tabular}


Tabela 5.12: Valores médios e desvios padrões para latência do Cenário A em segundos

\begin{tabular}{cccc}
\hline Falhas $(\%)$ & $\begin{array}{c}\text { Tempo de } \\
\text { pausa }(\mathrm{s})\end{array}$ & AODV $+\mathrm{G}+\mathrm{Q}$ & AODV $+\mathrm{G}+\mathrm{Q}+\mathrm{PWSave}$ \\
\hline 7,5 & 0 & $2,47 \pm 0,04$ & $3,14 \pm 0,04$ \\
7,5 & 50 & $2,49 \pm 0,04$ & $4,86 \pm 0,03$ \\
7,5 & 100 & $2,49 \pm 0,05$ & $4,69 \pm 0,04$ \\
7,5 & 150 & $2,47 \pm 0,05$ & $4,96 \pm 0,05$ \\
7,5 & 200 & $2,47 \pm 0,06$ & $4,89 \pm 0,04$ \\
7,5 & 250 & $2,47 \pm 0,07$ & $4,57 \pm 0,06$ \\
15 & 0 & $2,88 \pm 0,04$ & $3,39 \pm 0,03$ \\
15 & 50 & $2,95 \pm 0,04$ & $3,66 \pm 0,05$ \\
15 & 100 & $2,93 \pm 0,06$ & $3,53 \pm 0,05$ \\
15 & 150 & $2,98 \pm 0,04$ & $3,71 \pm 0,04$ \\
15 & 200 & $2,96 \pm 0,07$ & $3,78 \pm 0,06$ \\
15 & 250 & $2,89 \pm 0,06$ & $3,52 \pm 0,03$ \\
22,5 & 0 & $3,38 \pm 0,05$ & $4,21 \pm 0,04$ \\
22,5 & 50 & $3,39 \pm 0,05$ & $5,11 \pm 0,06$ \\
22,5 & 100 & $3,39 \pm 0,06$ & $4,97 \pm 0,04$ \\
22,5 & 150 & $3,47 \pm 0,04$ & $5,08 \pm 0,05$ \\
22,5 & 200 & $3,30 \pm 0,06$ & $4,80 \pm 0,04$ \\
22,5 & 250 & $3,36 \pm 0,07$ & $4,64 \pm 0,05$ \\
30 & 0 & $3,95 \pm 0,04$ & $4,49 \pm 0,03$ \\
30 & 50 & $3,95 \pm 0,04$ & $5,08 \pm 0,05$ \\
30 & 100 & $3,95 \pm 0,07$ & $4,84 \pm 0,04$ \\
30 & 150 & $3,94 \pm 0,04$ & $4,99 \pm 0,06$ \\
30 & 200 & $3,92 \pm 0,06$ & $4,92 \pm 0,04$ \\
30 & 250 & $3,97 \pm 0,06$ & $4,77 \pm 0,05$ \\
\hline & & & \\
\hline
\end{tabular}


Tabela 5.13: Valores médios e desvios padrões para latência do Cenário B em segundos

\begin{tabular}{cccc}
\hline Falhas $(\%)$ & $\begin{array}{c}\text { Tempo de } \\
\text { pausa }(\mathrm{s})\end{array}$ & AODV $+\mathrm{G}+\mathrm{Q}$ & AODV $+\mathrm{G}+\mathrm{Q}+\mathrm{PWSave}$ \\
\hline 7,5 & 0 & $2,48 \pm 0,04$ & $3,15 \pm 0,04$ \\
7,5 & 50 & $2,49 \pm 0,04$ & $4,86 \pm 0,04$ \\
7,5 & 100 & $2,49 \pm 0,04$ & $4,53 \pm 0,05$ \\
7,5 & 150 & $2,47 \pm 0,05$ & $4,96 \pm 0,05$ \\
7,5 & 200 & $2,47 \pm 0,05$ & $4,92 \pm 0,04$ \\
7,5 & 250 & $2,45 \pm 0,05$ & $4,55 \pm 0,05$ \\
15 & 0 & $2,88 \pm 0,04$ & $3,39 \pm 0,03$ \\
15 & 50 & $2,95 \pm 0,05$ & $3,66 \pm 0,04$ \\
15 & 100 & $2,93 \pm 0,06$ & $3,53 \pm 0,05$ \\
15 & 150 & $2,97 \pm 0,04$ & $3,70 \pm 0,05$ \\
15 & 200 & $2,94 \pm 0,05$ & $3,76 \pm 0,05$ \\
15 & 250 & $2,87 \pm 0,06$ & $3,50 \pm 0,05$ \\
22,5 & 0 & $3,33 \pm 0,05$ & $4,16 \pm 0,04$ \\
22,5 & 50 & $3,39 \pm 0,05$ & $5,11 \pm 0,05$ \\
22,5 & 100 & $3,39 \pm 0,05$ & $4,97 \pm 0,04$ \\
22,5 & 150 & $3,42 \pm 0,04$ & $5,03 \pm 0,05$ \\
22,5 & 200 & $3,30 \pm 0,04$ & $4,80 \pm 0,05$ \\
22,5 & 250 & $3,36 \pm 0,05$ & $4,64 \pm 0,05$ \\
30 & 0 & $3,95 \pm 0,04$ & $4,49 \pm 0,04$ \\
30 & 50 & $3,95 \pm 0,05$ & $5,08 \pm 0,05$ \\
30 & 100 & $3,94 \pm 0,07$ & $4,83 \pm 0,05$ \\
30 & 150 & $3,94 \pm 0,04$ & $4,99 \pm 0,04$ \\
30 & 200 & $3,94 \pm 0,05$ & $4,85 \pm 0,05$ \\
30 & 250 & $3,96 \pm 0,06$ & $4,76 \pm 0,05$ \\
\hline & & & \\
\hline
\end{tabular}


Tabela 5.14: Valores médios e desvios padrões para vazão do Cenário A em bits/seg.

\begin{tabular}{cccc}
\hline Falhas $(\%)$ & $\begin{array}{c}\text { Tempo de } \\
\text { pausa }(\mathrm{s})\end{array}$ & AODV $+\mathrm{G}+\mathrm{Q}$ & AODV $+\mathrm{G}+\mathrm{Q}+\mathrm{PWSave}$ \\
\hline 7,5 & 0 & $197812,80 \pm 499,51$ & $181679,67 \pm 578,99$ \\
7,5 & 50 & $130128,40 \pm 501,38$ & $125999,33 \pm 563,12$ \\
7,5 & 100 & $130895,07 \pm 500,78$ & $108142,63 \pm 587,67$ \\
7,5 & 150 & $136998,60 \pm 598,16$ & $114176,67 \pm 599,02$ \\
7,5 & 200 & $193782,83 \pm 498,56$ & $189464,04 \pm 585,43$ \\
7,5 & 250 & $197903,41 \pm 500,45$ & $178530,41 \pm 623,07$ \\
15 & 0 & $218766,10 \pm 570,58$ & $209128,15 \pm 601,13$ \\
15 & 50 & $129364,60 \pm 545,33$ & $124601,64 \pm 598,12$ \\
15 & 100 & $149833,23 \pm 503,67$ & $143769,68 \pm 593,33$ \\
15 & 150 & $145348,10 \pm 564,37$ & $139191,52 \pm 598,76$ \\
15 & 200 & $162974,29 \pm 550,34$ & $155809,31 \pm 601,22$ \\
15 & 250 & $176587,79 \pm 498,06$ & $167650,89 \pm 599,02$ \\
22,5 & 0 & $178438,40 \pm 491,33$ & $171560,10 \pm 601,02$ \\
22,5 & 50 & $125201,15 \pm 525,85$ & $119556,98 \pm 569,27$ \\
22,5 & 100 & $130544,91 \pm 502,09$ & $116271,66 \pm 585,04$ \\
22,5 & 150 & $127861,37 \pm 559,75$ & $123001,11 \pm 597,27$ \\
22,5 & 200 & $146988,34 \pm 499,06$ & $125492,9 \pm 587,87$ \\
22,5 & 250 & $160982,18 \pm 510,56$ & $158057,89 \pm 562,12$ \\
30 & 0 & $132825,90 \pm 526,80$ & $123912,96 \pm 591,01$ \\
30 & 50 & $125972,51 \pm 508,07$ & $121273,62 \pm 601,00$ \\
30 & 100 & $128031,14 \pm 511,34$ & $121438,38 \pm 598,13$ \\
30 & 150 & $129395,40 \pm 526,67$ & $124747,26 \pm 597,33$ \\
30 & 200 & $127983,26 \pm 478,90$ & $125841,57 \pm 600,55$ \\
30 & 250 & $133049,37 \pm 498,67$ & $132040,59 \pm 592,13$ \\
& & & \\
\hline
\end{tabular}


Tabela 5.15: Valores médios e desvios padrões para vazão do Cenário B em bits/seg.

\begin{tabular}{cccc}
\hline Falhas $(\%)$ & $\begin{array}{c}\text { Tempo de } \\
\text { pausa }(\mathrm{s})\end{array}$ & AODV $+\mathrm{G}+\mathrm{Q}$ & AODV $+\mathrm{G}+\mathrm{Q}+\mathrm{PWSave}$ \\
\hline 7,5 & 0 & $198823,43 \pm 456,34$ & $182690,30 \pm 538,69$ \\
7,5 & 50 & $130117,36 \pm 544,12$ & $125988,29 \pm 534,45$ \\
7,5 & 100 & $136876,34 \pm 531,74$ & $114123,90 \pm 512,45$ \\
7,5 & 150 & $136976,12 \pm 543,16$ & $114154,19 \pm 567,12$ \\
7,5 & 200 & $164778,11 \pm 465,35$ & $160459,32 \pm 587,84$ \\
7,5 & 250 & $167941,98 \pm 512,45$ & $148568,98 \pm 601,90$ \\
15 & 0 & $218776,45 \pm 512,08$ & $209138,50 \pm 615,34$ \\
15 & 50 & $130359,13 \pm 516,96$ & $125596,17 \pm 549,09$ \\
15 & 100 & $149825,75 \pm 534,56$ & $143762,20 \pm 566,89$ \\
15 & 150 & $143352,45 \pm 535,11$ & $137195,87 \pm 578,11$ \\
15 & 200 & $162967,46 \pm 578,14$ & $155802,48 \pm 605,78$ \\
15 & 250 & $176576,87 \pm 512,87$ & $167639,97 \pm 535,99$ \\
22,5 & 0 & $178429,22 \pm 502,31$ & $171550,92 \pm 623,14$ \\
22,5 & 50 & $127215,35 \pm 566,09$ & $121571,18 \pm 549,35$ \\
22,5 & 100 & $131526,64 \pm 523,33$ & $117253,39 \pm 531,14$ \\
22,5 & 150 & $129858,25 \pm 522,65$ & $124997,99 \pm 566,87$ \\
22,5 & 200 & $146965,17 \pm 433,12$ & $125469,73 \pm 567,98$ \\
22,5 & 250 & $163976,65 \pm 521,57$ & $161052,36 \pm 559,37$ \\
30 & 0 & $128844,15 \pm 567,22$ & $119931,21 \pm 557,87$ \\
30 & 50 & $125969,01 \pm 512,37$ & $121270,12 \pm 612,09$ \\
30 & 100 & $128014,16 \pm 524,14$ & $121421,40 \pm 543,26$ \\
30 & 150 & $129387,66 \pm 536,97$ & $124739,52 \pm 567,97$ \\
30 & 200 & $127936,64 \pm 498,20$ & $125794,95 \pm 605,76$ \\
30 & 250 & $130087,07 \pm 476,90$ & $129078,29 \pm 578,98$ \\
& & & \\
\hline
\end{tabular}


Tabela 5.16: Valores médios e desvios padrões para RREQ do Cenário A

\begin{tabular}{cccc}
\hline Falhas $(\%)$ & $\begin{array}{c}\text { Tempo de } \\
\text { pausa }(\mathrm{s})\end{array}$ & AODV $+\mathrm{G}+\mathrm{Q}$ & $\mathrm{AODV}+\mathrm{G}+\mathrm{Q}+\mathrm{PWSave}$ \\
\hline 7,5 & 0 & $85994,80 \pm 4341,84$ & $87492,43 \pm 4220,34$ \\
7,5 & 50 & $104356,45 \pm 4754,46$ & $111819,09 \pm 4330,78$ \\
7,5 & 100 & $102357,34 \pm 4951,69$ & $107890,88 \pm 4229,06$ \\
7,5 & 150 & $88799,36 \pm 5569,33$ & $92961,12 \pm 4450,87$ \\
7,5 & 200 & $98991,55 \pm 4908,64$ & $102754,77 \pm 5012,45$ \\
7,5 & 250 & $99994,80 \pm 4548,83$ & $104106,09 \pm 4556,70$ \\
15 & 0 & $49834,92 \pm 5206,41$ & $52283,99 \pm 4667,50$ \\
15 & 50 & $178864,20 \pm 4520,05$ & $188733,77 \pm 4778,44$ \\
15 & 100 & $149943,34 \pm 4373,37$ & $157150,33 \pm 4987,50$ \\
15 & 150 & $114351,00 \pm 4998,68$ & $118854,66 \pm 4443,69$ \\
15 & 200 & $121178,56 \pm 4164,55$ & $125795,94 \pm 4779,09$ \\
15 & 250 & $113403,18 \pm 4733,03$ & $117824,28 \pm 4880,34$ \\
22,5 & 0 & $60364,98 \pm 5198,81$ & $62504,73 \pm 5010,23$ \\
22,5 & 50 & $209723,80 \pm 5295,13$ & $219657,23 \pm 4980,78$ \\
22,5 & 100 & $180005,57 \pm 4295,86$ & $187042,45 \pm 4778,70$ \\
22,5 & 150 & $136405,60 \pm 4741,64$ & $141674,19 \pm 4872,12$ \\
22,5 & 200 & $146053,55 \pm 4147,53$ & $151656,89 \pm 4890,33$ \\
22,5 & 250 & $135382,76 \pm 4440,47$ & $140185,84 \pm 4479,23$ \\
30 & 0 & $72647,43 \pm 4851,00$ & $75243,89 \pm 4558,45$ \\
30 & 50 & $275650,50 \pm 4583,34$ & $286248,88 \pm 4665,31$ \\
30 & 100 & $216286,67 \pm 5948,16$ & $225025,99 \pm 5012,37$ \\
30 & 150 & $184230,50 \pm 5122,19$ & $190265,36 \pm 5001,01$ \\
30 & 200 & $171575,69 \pm 7664,08$ & $506370,42 \pm 4997,40$ \\
30 & 250 & $162484,33 \pm 8564,10$ & $170894,29 \pm 4675,40$ \\
\hline & & & \\
\hline & & & \\
& 0 & &
\end{tabular}


Tabela 5.17: Valores médios e desvios padrões para RREQ do Cenário B

\begin{tabular}{cccc}
\hline Falhas $(\%)$ & $\begin{array}{c}\text { Tempo de } \\
\text { pausa }(\mathrm{s})\end{array}$ & AODV $+\mathrm{G}+\mathrm{Q}$ & AODV $+\mathrm{G}+\mathrm{Q}+\mathrm{PWSave}$ \\
\hline 7,5 & 0 & $85994,61 \pm 4323,08$ & $87567,78 \pm 4220,54$ \\
7,5 & 50 & $104367,23 \pm 4754,12$ & $111654,23 \pm 4312,04$ \\
7,5 & 100 & $102362,33 \pm 4951,01$ & $107657,31 \pm 4243,33$ \\
7,5 & 150 & $98978,44 \pm 5503,12$ & $102917,76 \pm 4467,31$ \\
7,5 & 200 & $99981,67 \pm 4911,01$ & $103787,03 \pm 5034,65$ \\
7,5 & 250 & $99994,80 \pm 4550,12$ & $104212,15 \pm 4534,18$ \\
15 & 0 & $49827,75 \pm 5212,15$ & $52233,13 \pm 4654,13$ \\
15 & 50 & $178855,03 \pm 4529,13$ & $188613,14 \pm 4752,04$ \\
15 & 100 & $149938,12 \pm 4387,11$ & $157146,24 \pm 4987,50$ \\
15 & 150 & $114322,09 \pm 4970,04$ & $118633,45 \pm 4404,33$ \\
15 & 200 & $119186,73 \pm 4171,62$ & $123641,13 \pm 4734,21$ \\
15 & 250 & $113431,86 \pm 4711,54$ & $117713,76 \pm 4856,02$ \\
22,5 & 0 & $60358,13 \pm 5156,34$ & $62512,08 \pm 5027,83$ \\
22,5 & 50 & $209734,78 \pm 5256,65$ & $219631,14 \pm 4976,54$ \\
22,5 & 100 & $180033,91 \pm 4265,23$ & $187032,13 \pm 4767,34$ \\
22,5 & 150 & $136489,33 \pm 4734,12$ & $141643,32 \pm 4876,88$ \\
22,5 & 200 & $146044,66 \pm 4133,09$ & $151623,51 \pm 4878,24$ \\
22,5 & 250 & $135397,02 \pm 4426,74$ & $140173,15 \pm 4461,16$ \\
30 & 0 & $72634,99 \pm 4843,12$ & $75203,33 \pm 4534,65$ \\
30 & 50 & $275634,05 \pm 4555,02$ & $286232,18 \pm 4643,11$ \\
30 & 100 & $216286,67 \pm 5933,16$ & $225012,43 \pm 5003,00$ \\
30 & 150 & $184269,99 \pm 5112,08$ & $190243,12 \pm 5032,44$ \\
30 & 200 & $170556,04 \pm 7644,12$ & $505122,32 \pm 4944,09$ \\
30 & 250 & $162464,04 \pm 8509,44$ & $170854,56 \pm 4654,33$ \\
\hline & & & \\
\hline
\end{tabular}


Tabela 5.18: Valores médios e desvios padrões para consumo de energia do Cenário A em mWhr

\begin{tabular}{cccc}
\hline Falhas $(\%)$ & $\begin{array}{c}\text { Tempo de } \\
\text { pausa }(\mathrm{s})\end{array}$ & AODV $+\mathrm{G}+\mathrm{Q}$ & AODV $+\mathrm{G}+\mathrm{Q}+\mathrm{PWSave}$ \\
\hline 7,5 & 0 & $6003,99 \pm 0,49$ & $5372,23 \pm 0,43$ \\
7,5 & 50 & $6004,52 \pm 0,50$ & $5394,07 \pm 0,39$ \\
7,5 & 100 & $6003,26 \pm 0,47$ & $5382,15 \pm 0,45$ \\
7,5 & 150 & $6003,12 \pm 0,49$ & $5378,34 \pm 0,43$ \\
7,5 & 200 & $6002,54 \pm 0,49$ & $5377,03 \pm 0,29$ \\
7,5 & 250 & $6003,77 \pm 0,43$ & $5373,78 \pm 0,49$ \\
15 & 0 & $6004,40 \pm 0,47$ & $5371,09 \pm 0,49$ \\
15 & 50 & $6004,63 \pm 0,49$ & $5396,11 \pm 0,45$ \\
15 & 100 & $6003,25 \pm 0,44$ & $5379,33 \pm 0,43$ \\
15 & 150 & $6003,14 \pm 0,53$ & $5375,42 \pm 0,40$ \\
15 & 200 & $6003,67 \pm 0,44$ & $5373,21 \pm 0,39$ \\
15 & 250 & $6003,98 \pm 0,45$ & $5370,02 \pm 0,51$ \\
22,5 & 0 & $6000,87 \pm 0,51$ & $5367,35 \pm 0,45$ \\
22,5 & 50 & $6000,51 \pm 0,50$ & $5393,57 \pm 0,53$ \\
22,5 & 100 & $5999,67 \pm 0,47$ & $5378,16 \pm 0,45$ \\
22,5 & 150 & $6000,00 \pm 0,43$ & $5373,81 \pm 0,43$ \\
22,5 & 200 & $5999,66 \pm 0,50$ & $5369,02 \pm 0,45$ \\
22,5 & 250 & $6001,04 \pm 0,42$ & $5366,11 \pm 0,53$ \\
30 & 0 & $5998,70 \pm 0,40$ & $5365,74 \pm 0,50$ \\
30 & 50 & $5998,54 \pm 0,41$ & $5391,53 \pm 0,43$ \\
30 & 100 & $5998,59 \pm 0,48$ & $5375,40 \pm 0,51$ \\
30 & 150 & $5998,62 \pm 0,47$ & $5371,18 \pm 0,49$ \\
30 & 200 & $5998,80 \pm 0,48$ & $5366,53 \pm 0,49$ \\
30 & 250 & $5999,56 \pm 0,40$ & $5364,25 \pm 0,54$ \\
\hline
\end{tabular}




\section{Anexo C - Código fonte do algoritmo Gossip}

\section{Código original do AODV no Glomosim adaptado para esta dissertação}

\section{$\operatorname{aodv.h}$}

1

5 typedef struct

6\{

8

9

10

14 typedef struct glomo_network_aodv_str

15

16

18 GlomoRoutingAodv;

\section{aodv.pc}

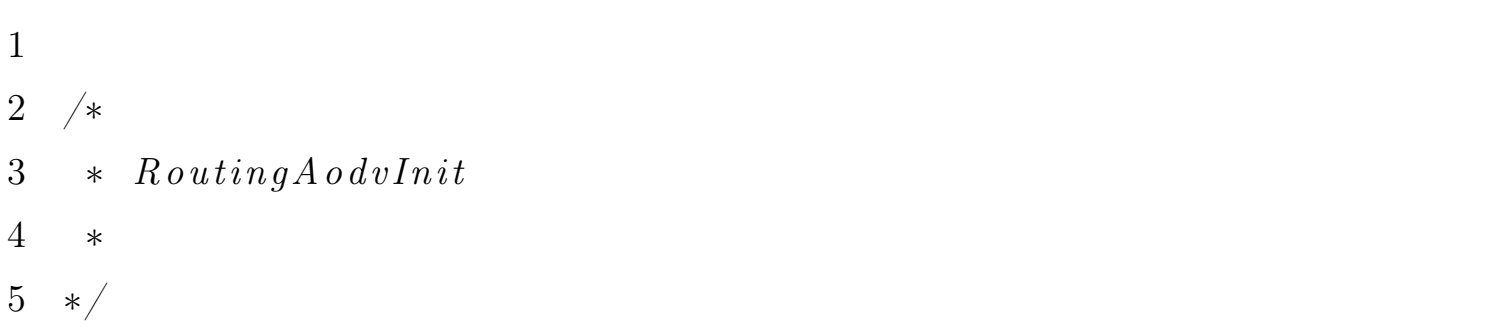


7 // Função de Inicialização para o protocolo AODV

8 // com a aplicação do Gossip

9

10 void RoutingAodvInit (

11 GlomoNode *node,

12 GlomoRoutingAodv **aodvPtr,

13 const GlomoNodeInput *nodeInput)

14\{

15

17 \} * RoutingAodvInit */

18

19

20

$21 / *$

$22 *$ RoutingAodvFinalize

$23 *$

$24 * /$

25

26 // Função chamada no fim da simulação

27 // para gerar relatório dos dados da vizinhança

$28 / /$ do Gossip

29

30 void RoutingAodvFinalize (GlomoNode *node)

31\{

32

//imprime lista de vizinhança de cada nó

34 current $=$ aodv $\rightarrow$ completeNbrTable.head;

35

36

37

while (current $!=$ NULL) \{

printf ( $\backslash \% \mathrm{~d}_{\llcorner} "$, current $\rightarrow$ destAddr $)$;

current $=$ current $\rightarrow$ next ;

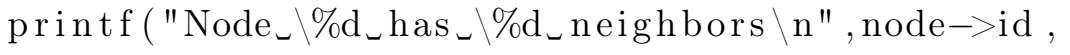
aodv $\rightarrow$ completeNbrTable.size );

$41\}$ /* RoutingAodvFinalize */ 


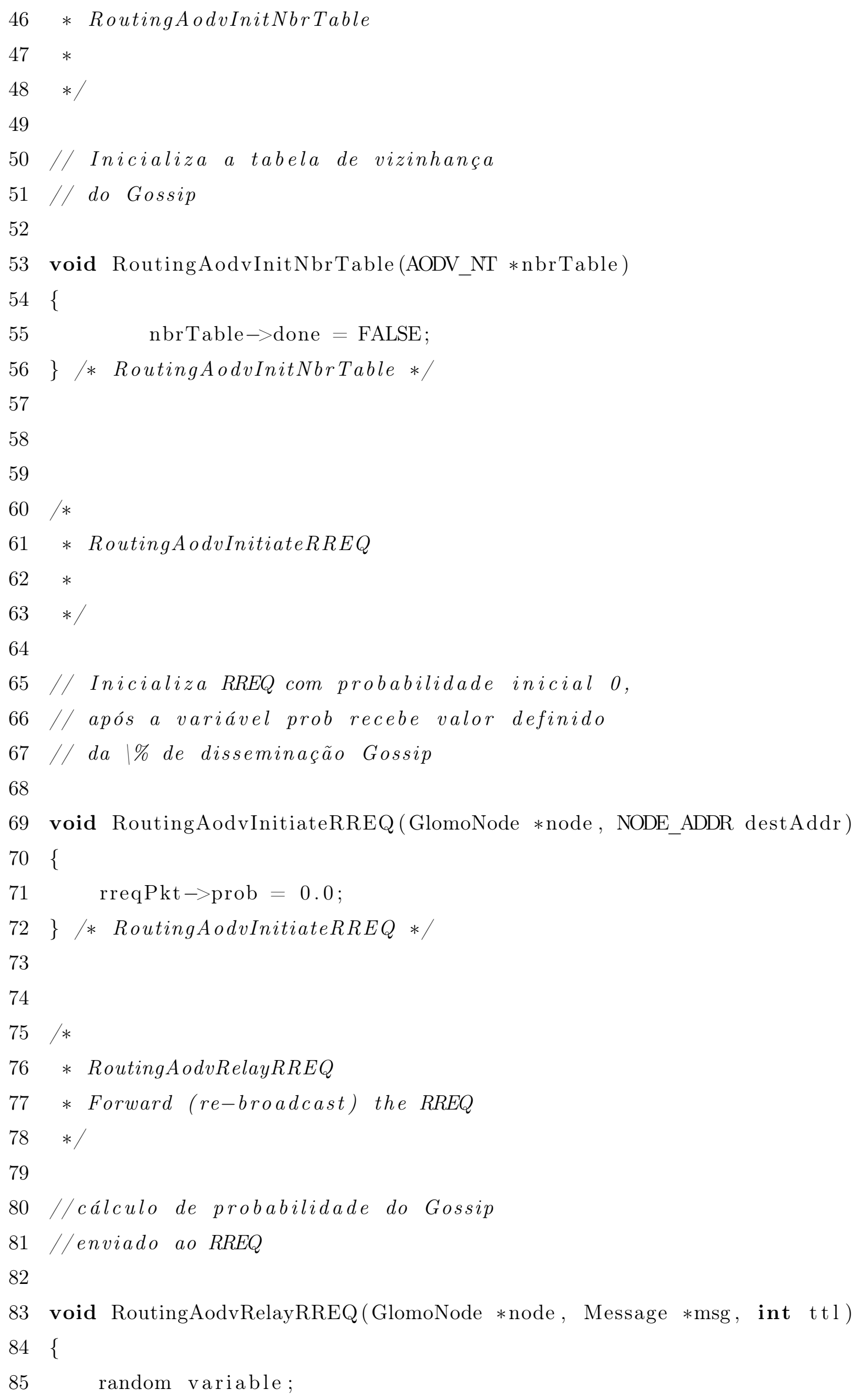




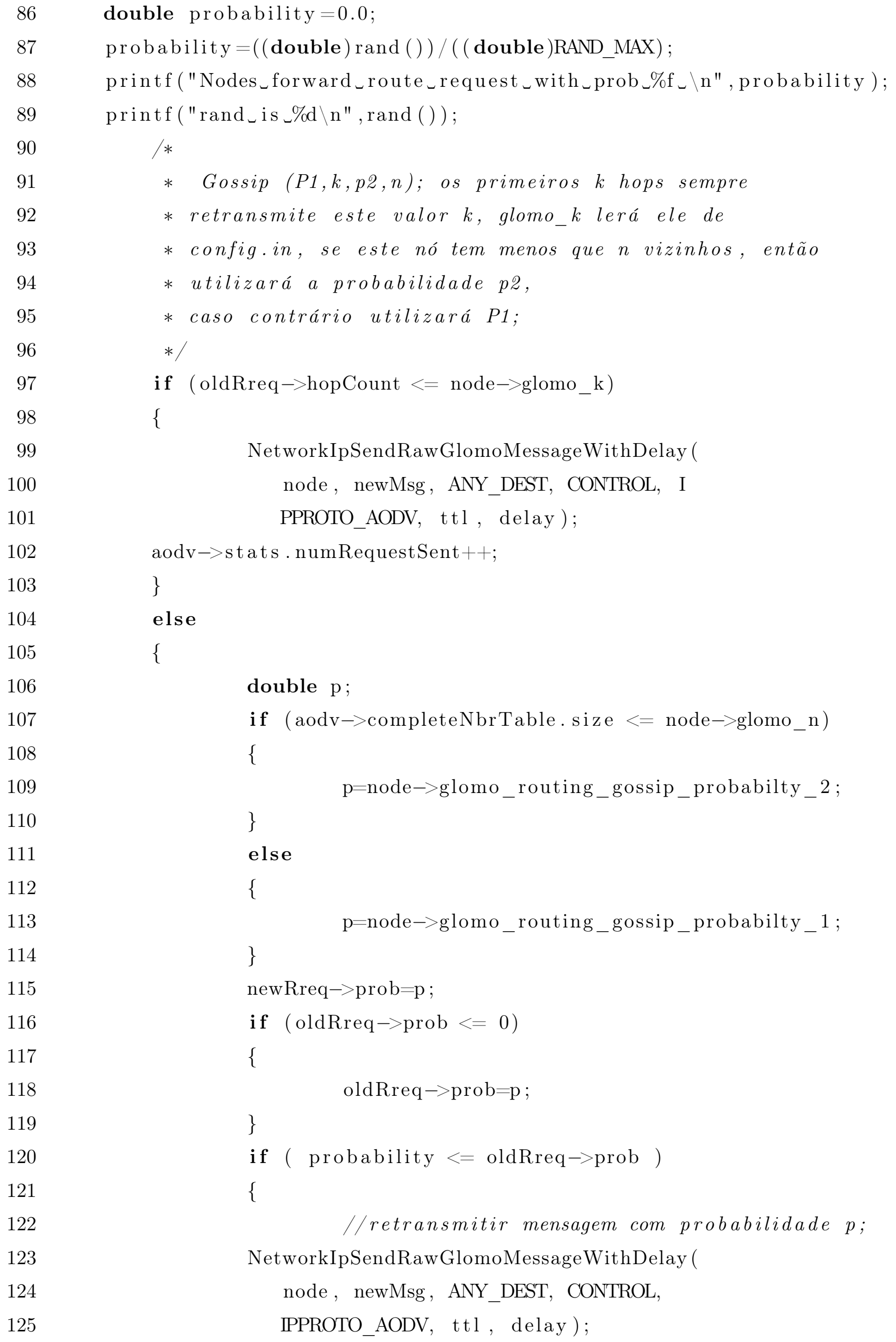


126

127

128

129

130 aodv $\rightarrow$ stats . numRequestSent ++ ;

\}

\}

GLOMO_MsgFree(node, msg);

\section{Código original do DSR no Glomosim adaptado para esta dissertação}

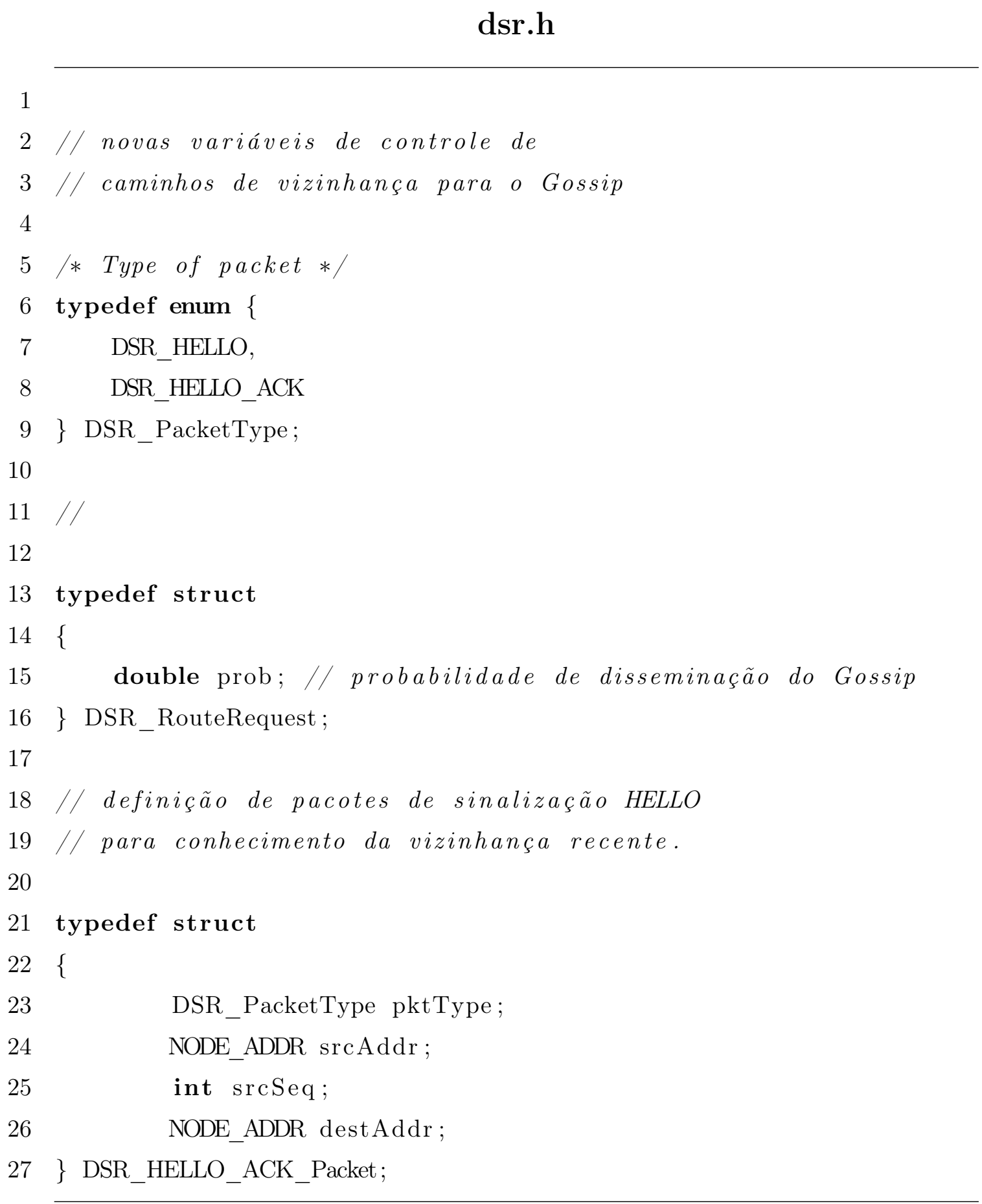




\section{dsr.pc}

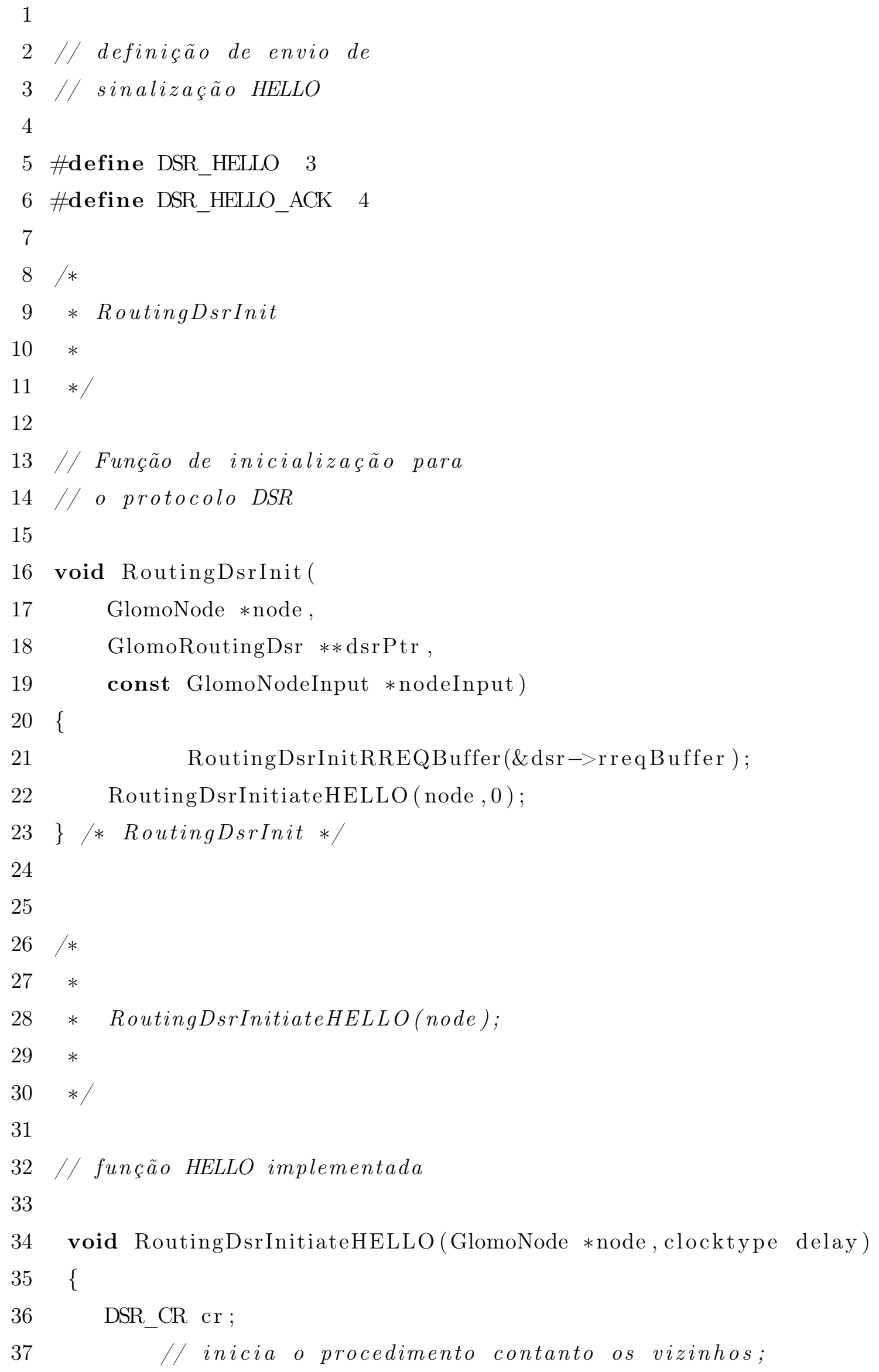


41
GlomoNetworkIp $*$ ipLayer $=($ GlomoNetworkIp $*)$ node $->$ networkData . networkVar;

GlomoRoutingDsr $* \mathrm{dsr}=($ GlomoRoutingDsr $*)$ ipLayer $\rightarrow$ routingProtocol;

Message *newMsg;

DSR_HELLO_Packet $*$ helloPkt;

char *pktPtr;

int pktSize $=$ sizeof(DSR_HELLO_Packet $)$;

int $\mathrm{ttl}$;

newMsg = GLOMO_MsgAlloc(node, GLOMO_MAC_LAYER,

0 , MSG_MAC_FromNetwork);

GLOMO_MsgPacketAlloc(node, newMsg, pktSize); pktPtr $=($ char $*)$ GLOMO_MsgReturnPacket (newMsg $)$; node, newMsg, ANY_DEST, CONTROL, IPPROTO_DSR, ttl, delay);

\}

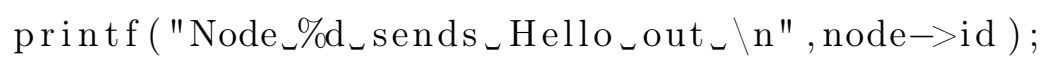

// timer para a tablea de vizinhos, reinicia

// a tabela depois de tempo esgotado

77 // por causa da mobilidade; 


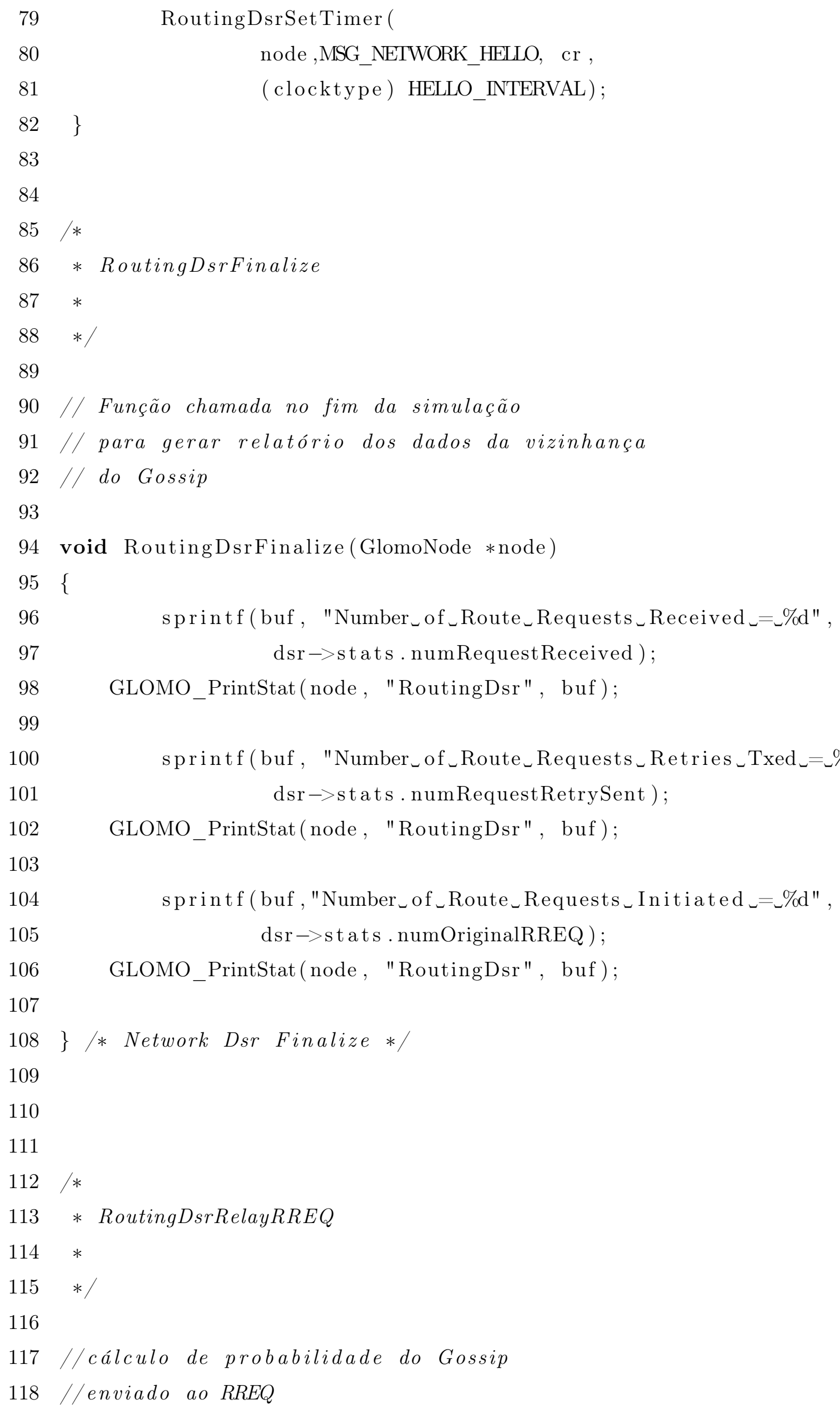


119

120 void RoutingDsrRelayRREQ(GlomoNode *node, Message *msg, int ttl)

121\{

122 probability $=(($ double $)$ rand ()$) /(($ double $)$ RAND_MAX $)$;

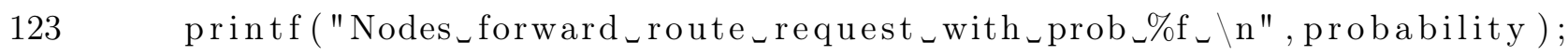

$124 \quad$ printf ( "rand is $\left._{\longleftarrow} \% \mathrm{~d} \backslash \mathrm{n} ", \operatorname{rand}()\right)$;

125

126

127

128

129

130

131

132

133

134

135

136

137

138

139

140

141

142

143

144

145

146

147

148

149

150

151

152

153

154

155

156

157

double $\mathrm{p}$;

NetworkIpSendRawGlomoMessageWithDelay ( node, newMsg, ANY_DEST, CONTROL, IPPROTO_DSR, ttl, delay);

dsr $\rightarrow$ stats . numRequestSent ++ ;

\}

else

\{

158

if $($ dsr $\rightarrow$ completeNbrTable.size $<=$ node $->$ glomo_n $)$ \{ $\mathrm{p}=$ node $->$ glomo_routing_gossip_probabilty_ $2 ;$

\}

else

\{

$\mathrm{p}=$ node $->$ glomo_routing_gossip_probabilty_ 1 ;

\}

newRreq $\rightarrow$ prob $=$ p;

if $($ oldRreq $->$ prob $<=0)$

\{

oldRreq $\rightarrow$ prob $=$ p ;

\}

if ( probability $<=$ oldRreq $->$ prob ) 
159

\{

160

//relay this message with probability $p$;

$161\} / *$ RoutingDsrRelayRREQ */ 


\section{Anexo D - Código fonte do sistema de Quorum}

\section{Código original do AODV adaptado para esta dissertação}

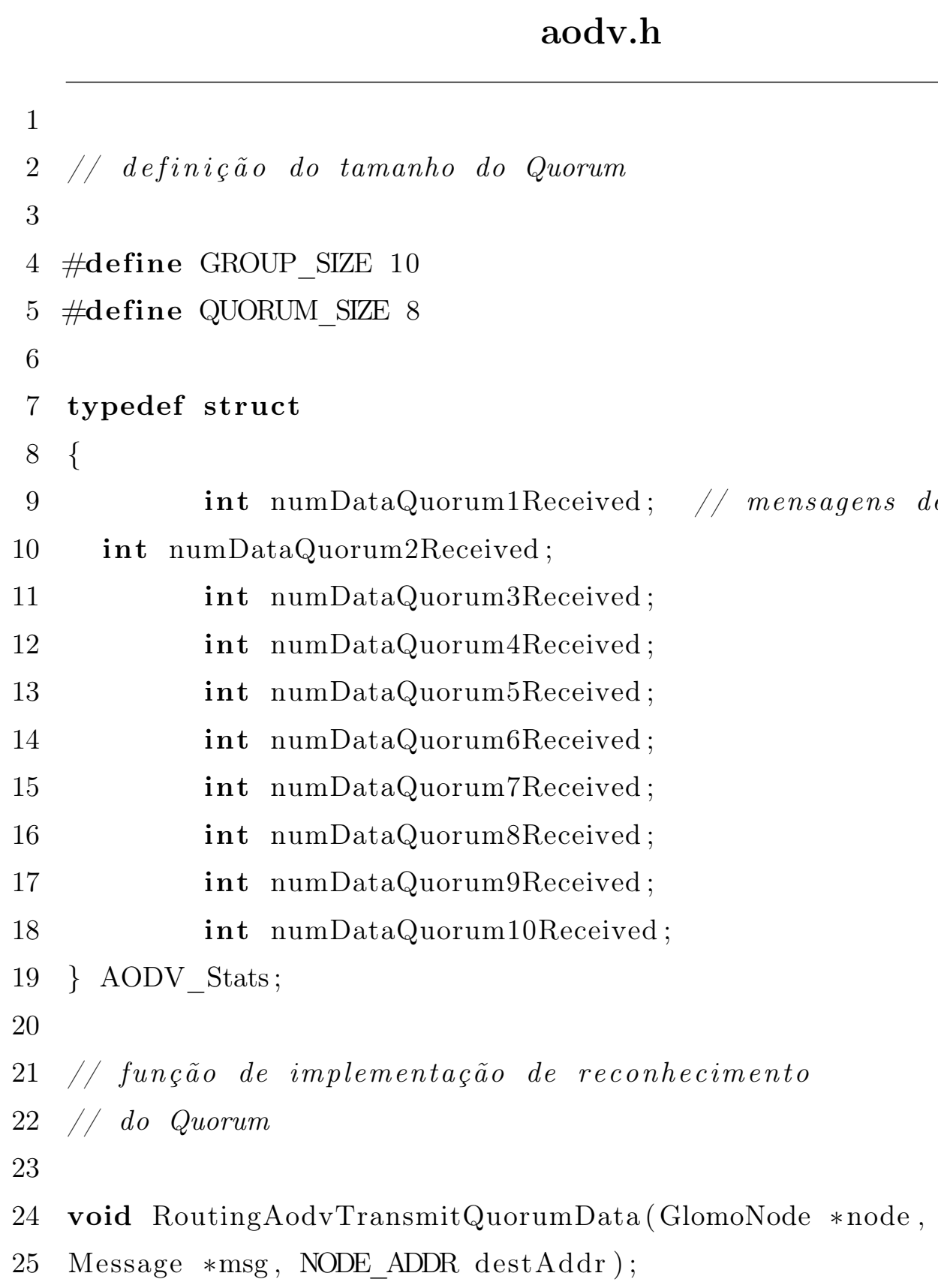




\section{aodv.pc}

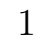

2 BOOL ls_on [80];

$3 / / 80$ nós dos quais 10 serão

4 //servidores backup para quorum

5

$6 / /$ int limite $=0$;

7

$8 / *$

$9 *$ RoutingAodvInit

$10 *$

11 * Função de inicialização do AODV

$12 * /$

13 void RoutingAodvInit (

14 GlomoNode *node,

15 GlomoRoutingAodv **aodvPtr,

16 const GlomoNodeInput *nodeInput)

17\{

18 GlomoRoutingAodv $* \operatorname{aodv}=$

19 (GlomoRoutingAodv *) checked_pc_malloc

$20 \quad(\operatorname{sizeof}($ GlomoRoutingAodv $))$;

$21 \quad(* \operatorname{aodvPtr})=\operatorname{aodv}$;

22 if $(\operatorname{aodv}=$ NULL $)$

23\{

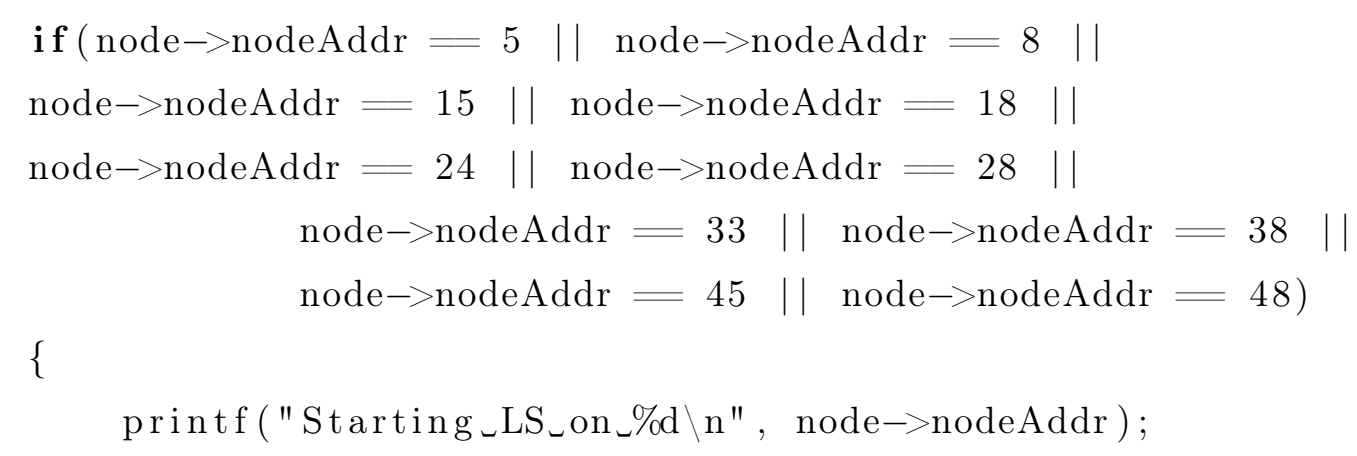


39

40

41

42

43

46

47

48

49

50

51

52

53

54

55

56

57

58

59

60

61

62

63

64

66 67

68

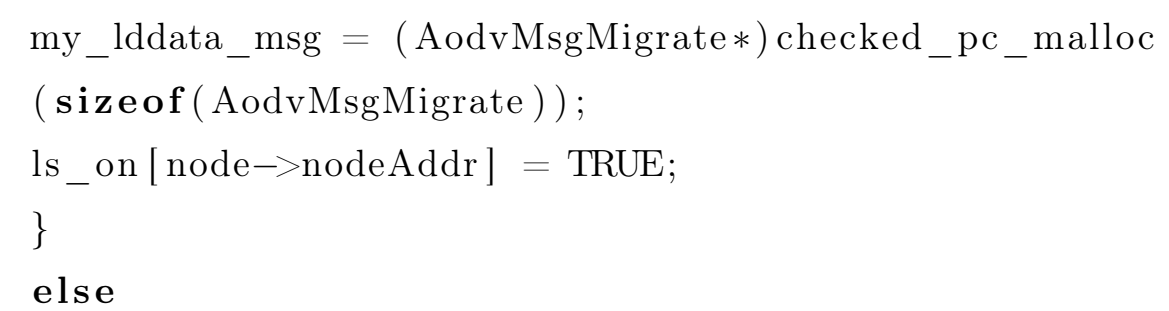
(1)

4

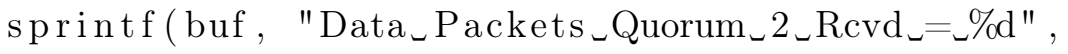
$($ aodv $\rightarrow$ stats . numDataQuorum2Received ) ); GLOMO_PrintStat(node, "RoutingAodv" , buf);

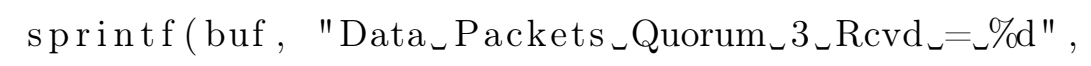
$($ aodv $\rightarrow$ stats.numDataQuorum3Received )); GLOMO_PrintStat(node, "RoutingAodv" , buf); $($ aodv $\rightarrow$ stats . numDataQuorum5Received ));

GLOMO_PrintStat(node, "RoutingAodv" , buf); 
79

80

81

82

83

84

100 \} /* RoutingAodvFinalize */

101

102

103

104

* RoutingAodvHandleData

$105 *$

$106 * /$

107

108 109

110 void RoutingAodvHandleData(GlomoNode *node,

111 Message $*$ msg, NODE_ADDR destAddr)

112\{

113

GLOMO_PrintStat (node, "RoutingAodv", buf);

GLOMO_PrintStat(node, "RoutingAodv", buf);

GLOMO_PrintStat(node, "RoutingAodv", buf);

GLOMO_PrintStat(node, "RoutingAodv", buf);

$$
\text { // armazenamento dos dados pelo Quorum }
$$

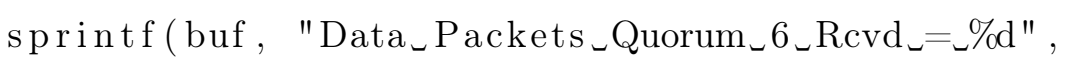
$($ aodv $\rightarrow$ stats . numDataQuorum6Received ));

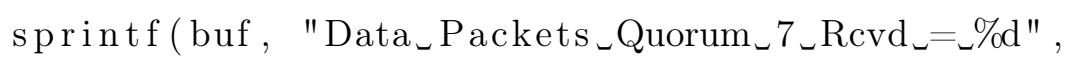$$
(\text { aodv } \rightarrow \text { stats . numDataQuorum7Received )); }
$$

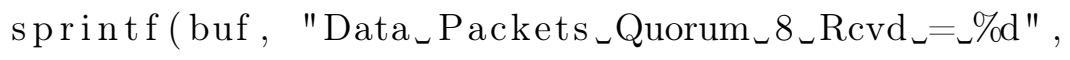
$($ aodv $\rightarrow$ stats . numDataQuorum8Received ));

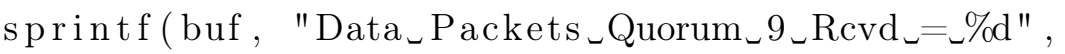
$($ aodv $\rightarrow$ stats . numDataQuorum9Received ));

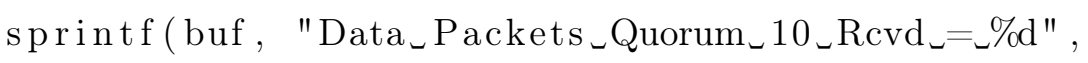
$($ aodv $\rightarrow$ stats . numDataQuorum10Received ) ); 


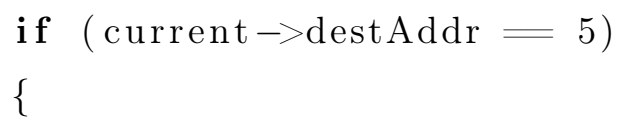

RoutingAodvTransmitQuorumData (node, msg, 8); aodv $\rightarrow$ stats . numDataReceived ++ ; aodv $\rightarrow$ stats. limite ++ ;

\}

else if (current $\rightarrow$ destAddr $=15)$

\{

RoutingAodvTransmitQuorumData (node, msg, 15); aodv $\rightarrow$ stats . numDataReceived ++ ; aodv $\rightarrow$ stats. limite ++ ;

\}

else if (current $\rightarrow$ destAddr $=18)$ \{

RoutingAodvTransmitQuorumData (node, msg, 18); aodv $\rightarrow$ stats . numDataReceived ++ ; aodv $\rightarrow$ stats . limite ++ ;

\}

else if (current $\rightarrow$ destAddr $=24$ ) \{ RoutingAodvTransmitQuorumData(node, msg, 24); aodv $\rightarrow$ stats . numDataReceived ++ ; aodv $\rightarrow$ stats . limite ++ ;

\}

else if (current $\rightarrow$ destAddr $=28$ )

\{ RoutingAodvTransmitQuorumData (node, msg, 28); aodv $\rightarrow$ stats . numDataReceived ++ ; aodv $\rightarrow$ stats. limite ++ ;

\} else if (current $\rightarrow$ destAddr $=33$ ) 
159

160

161

162

163

164

165

166

167

168

169

170

171

172

173

174

175

176

177

178

179

180

181

182

183

184

185

186

187

188

189

190

191

192

193

194

195 void Routing AodvInitStats (GlomoNode *node)

196

197

198

aodv $\rightarrow$ stats. limite ++ ;

\}

else if (current $\rightarrow$ destAddr $=38$ )

\{

aodv $\rightarrow$ stats . limite ++ ;

\}

else if (current $\rightarrow$ destAddr $=45)$

\{

aodv $\rightarrow$ stats. limite ++ ;

\}

else if (current $\rightarrow$ destAddr $=48$ )

\{

aodv $\rightarrow$ stats. limite ++ ;

\}

current $=$ current $\rightarrow$ next ;

\}

\}//if //

\}//if //

/* RoutingAodvHandleData */

* RoutingAodvInitStats

$*$

$* /$

//inicialização de variáveis do Quorum

\{

$\operatorname{aodv} \rightarrow$ stats. limite $=0$;

aodv $\rightarrow$ stats. numDataQuorum1Received $=0$;

RoutingAodvTransmitQuorumData(node, msg, 33 );

aodv $\rightarrow$ stats . numDataReceived ++ ;

RoutingAodvTransmitQuorumData (node, msg, 38); aodv $\rightarrow$ stats . numDataReceived ++ ;

RoutingAodvTransmitQuorumData (node, msg, 45);

aodv $\rightarrow$ stats . numDataReceived ++ ;

RoutingAodvTransmitQuorumData (node, msg, 48);

aodv $\rightarrow$ stats . numDataReceived ++ ; 


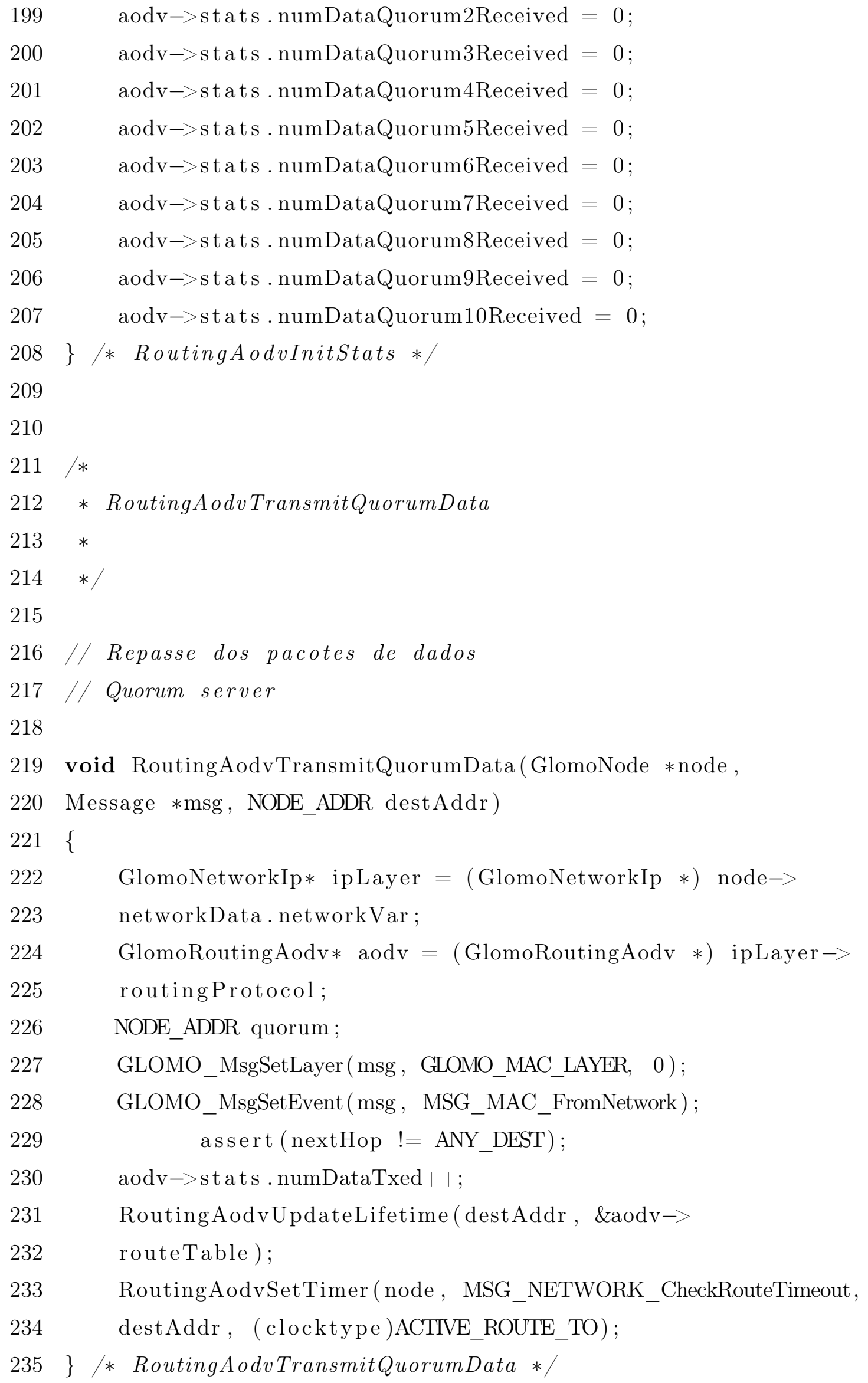




\section{Anexo E - Código fonte do sistema de Falhas}

\section{Código original do AODV adaptado para esta dissertação - Cenário A}

\section{$\operatorname{aodv} \cdot \mathrm{h}$}

1

2 // função para apagar dados do nó

3 // simulando a adormecimento

4

5 void AodvDeleteDataFaultA (GlomoNode *node, Message *msg,

6 NODE_ADDR destAddr, double prob);

7

$8 \backslash$ bend $\{$ lstlisting $\}$

9

$10 \backslash$ subsection $*\{\operatorname{aodv} \cdot \mathrm{pc}\}$

11

$12 \backslash$ begin $\{$ lstlisting $\}$

13

14

15

16

17

18

19 // função para apagar dados do nó

20 // simulando a adormecimento

21

22 void RoutingAodvInit (
25 \} /* RoutingAodvInit */

$$
\text { if timestamp }+=150\{/ / \text { cenário } A
$$

AodvDeleteDataFaultA (destAddr $\rightarrow$ current ,DATA);

26 
27

$$
28
$$

29

\section{0}$$
32
$$

\section{* AodvDeleteDataFaultA}

$* /$

BOOL AodvDeleteDataFaultA (NODE_ADDR destAddr,

AODV_DATA $*$ DATA, double prob) 
$\& \&($ DATA $>$ head $->$ destAddr $=74) \& \&($ DATA $>$ head $\rightarrow$ destAddr $=79))$ \{

toFree $=$ DATA $>$ head

DATA $>$ head $=$ toFree $\rightarrow$ next

pc_free (toFree);

$--($ DATA $>$ size $)$;

deleted = TRUE;

\}

$$
\text { \} }
$$

$/ /$ probabilidade de falha $=22,5 \mid \%$

$$
\text { else if prob }=22.5\{
$$

$(($ DATA $>$ head $->$ destAdd $r=1) \& \&($ DATA $>$ head $\rightarrow$ destAddr $=4)$ $\& \&($ DATA $>$ head $\rightarrow$ destAddr $=7) \& \&($ DATA $>$ head $\rightarrow$ destAddr $=11)$ $\& \&($ DATA $>$ head $\rightarrow$ destAddr $=14) \& \&($ DATA $>$ head $\rightarrow$ destAddr $=17)$ $\& \&($ DATA $>$ head $\rightarrow$ destAddr $=22) \& \&($ DATA $>$ head $\rightarrow$ destAddr $=26)$ $\& \&($ DATA $>$ head $\rightarrow$ destAdd $r=31) \& \&($ DATA $>$ head $\rightarrow$ destAddr $=34)$ $\& \&($ DATA $>$ head $\rightarrow$ destAddr $=39) \& \&($ DATA $>$ head $\rightarrow$ destAddr $=44)$ $\& \&($ DATA $>$ head $->$ destAddr $=47) \& \&($ DATA $>$ head $\rightarrow$ destAddr $=54)$ $\& \&($ DATA $>$ head $\rightarrow$ destAddr $=59)$ \&\& $($ DATA $>$ head $\rightarrow$ destAddr $=63)$ $\& \&($ DATA $>$ head $\rightarrow$ destAddr $=74) \& \&($ DATA $>$ head $\rightarrow$ destAddr $=79))$ \{ toFree $=$ DATA $>$ head

DATA $>$ head $=$ toFree $\rightarrow$ next pc_free(toFree); $-($ DATA $>$ size $)$; deleted = TRUE; \} \}

$/ /$ probabilidade de falh $a=30 \mid \%$

else if prob $=30\{$

$(($ DATA $>$ head $\rightarrow$ destAddr $=1)$ \&\& $($ DATA $>$ head $\rightarrow$ destAddr $=4)$ $\& \&($ DATA $>$ head $\rightarrow$ destAddr $=7) \& \&($ DATA $>$ head $\rightarrow$ destAddr $=9)$ $\& \&($ DATA $>$ head $\rightarrow$ destAddr $=11) \& \&($ DATA $>$ head $\rightarrow$ destAddr $=14)$ $\& \&($ DATA $>$ head $\rightarrow$ destAddr $=17) \& \&($ DATA $>$ head $\rightarrow$ destAddr $=19)$ $\& \&($ DATA $>$ head $\rightarrow$ destAddr $=22) \quad \& \&($ DATA $>$ head $\rightarrow$ destAddr $=26)$ $\& \&($ DATA $>$ head $\rightarrow$ destAddr $=29) \quad \& \&($ DATA $>$ head $\rightarrow$ destAddr $=31)$ 


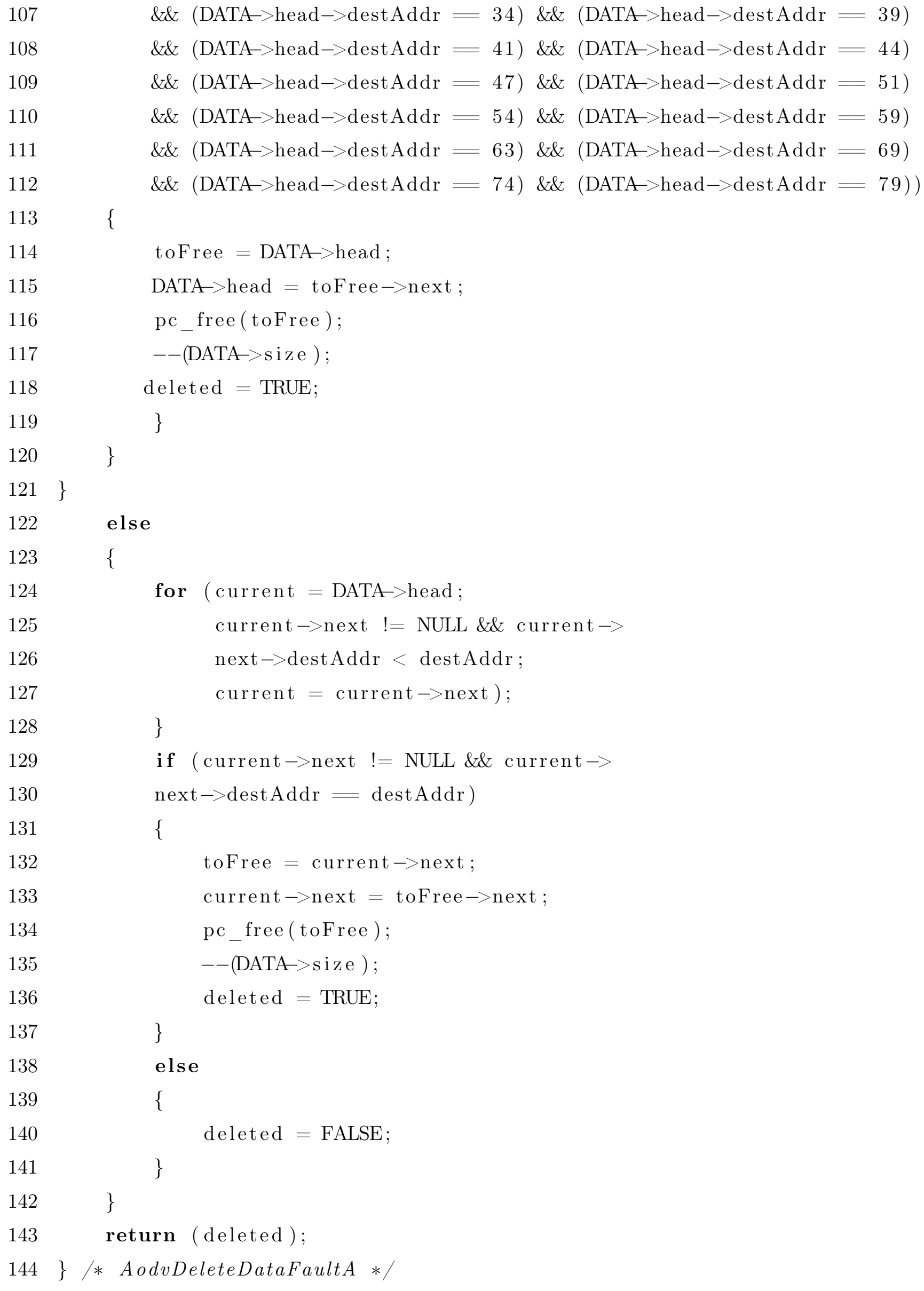




\section{Código original do AODV adaptado para esta dissertação - Cenário B}

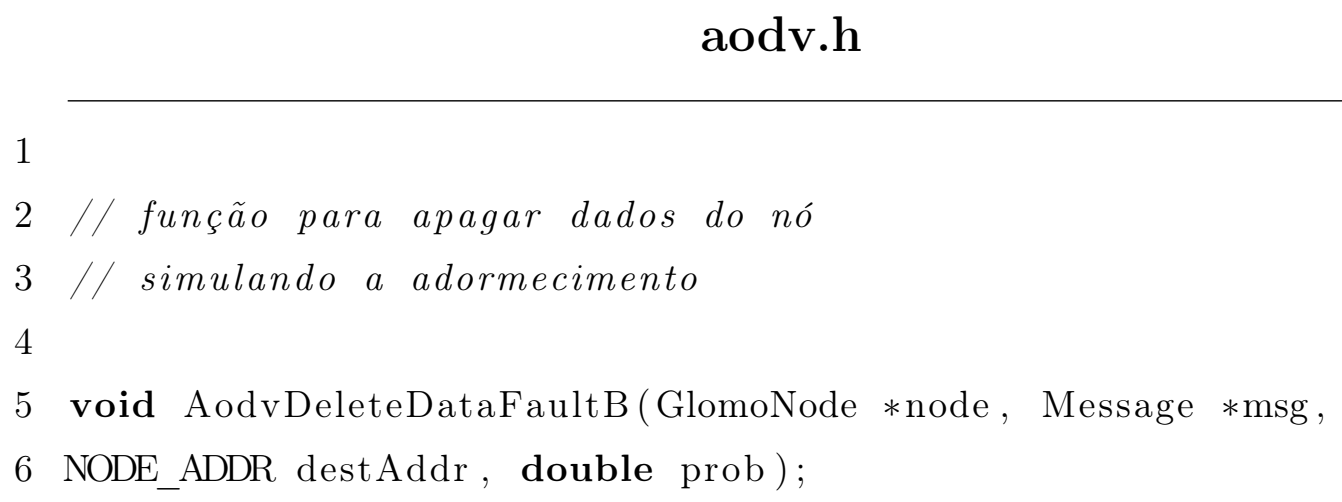

$\operatorname{aodv} \cdot \mathrm{pc}$

$2 / *$

$3 *$ RoutingAodvInit

$4 *$

$5 * /$

6

7 // função para apagar dados do nó

8 // simulando a adormecimento

9

10 void RoutingAodvinit ( current,DATA);

15 \} * RoutingAodvInit */

16

$18 *$ AodvDeleteDataFaultB

$19 * /$

20 BOOL AodvDeleteDataFaultB (NODE_ADDR destAddr,

21 AODV_DATA $*$ DATA, double prob)

22\{

23 AODV_DATA_Node $*$ toFree;

24 AODV_DATA_Node $*$ current;

25 double prob;

$26 \quad$ BOOL deleted; 


\section{time timestamp $=0$; \\ timestamp $=\operatorname{simclock}()$;}

for timestamp $<\mathrm{F} 1$

$$
\{
$$

$($ DATA $>$ size $=0)$;

deleted $=$ FALSE;

$$
\text { else if prob }=7.5\{
$$

// nós falhos selecionados pelo software $R$

$(($ DATA $>$ head $\rightarrow$ destAddr $=1) \& \&($ DATA $>$ head $\rightarrow$ destAddr $=14))$ \{

toFree $=$ DATA $>$ head

DATA $>$ head $=$ toFree $\rightarrow$ next ;

pc_free(toFree);

$--($ DATA $>$ size $)$;

deleted = TRUE;

\}

\}

$$
\text { else if prob }=15\{
$$

$(($ DATA $>$ head $->$ destAddr $=31) \& \&($ DATA $>$ head $\rightarrow$ destAddr $=39)$

$\& \&($ DATA $>$ head $\rightarrow$ destAddr $=47)$ \&\& $($ DATA $>$ head $\rightarrow$ destAddr $=54)$

$\& \&($ DATA $>$ head $\rightarrow$ destAddr $=63) \& \&($ DATA $>$ head $\rightarrow$ destAddr $=74)$

$\& \&($ DATA $>$ head $\rightarrow$ destAddr $=79))$

\{

toFree $=$ DATA $>$ head

DATA $>$ head $=$ toFree $\rightarrow$ next ;

pc_free(toFree);

$--($ DATA $>$ size $)$;

deleted = TRUE;

\}

$$
\text { \} }
$$

else if prob $=22.5\{$

$(($ DATA $>$ head $->$ destAddr $=31) \quad \& \&($ DATA $>$ head $->$ destAddr $=34)$ $\& \&($ DATA $>$ head $\rightarrow$ destAddr $=39) \& \&($ DATA $>$ head $\rightarrow$ destAddr $=44)$ $\& \&($ DATA $>$ head $\rightarrow$ destAddr $=47) \& \&($ DATA $>$ head $\rightarrow$ destAddr $=54)$ $\& \&($ DATA $>$ head $\rightarrow$ destAddr $=59) \& \&($ DATA $>$ head $\rightarrow$ destAddr $=63)$ $\& \&($ DATA $>$ head $->$ destAddr $=74) \& \&($ DATA $>$ head $\rightarrow$ destAddr $=79))$ \{ toFree $=$ DATA $>$ head $;$ 


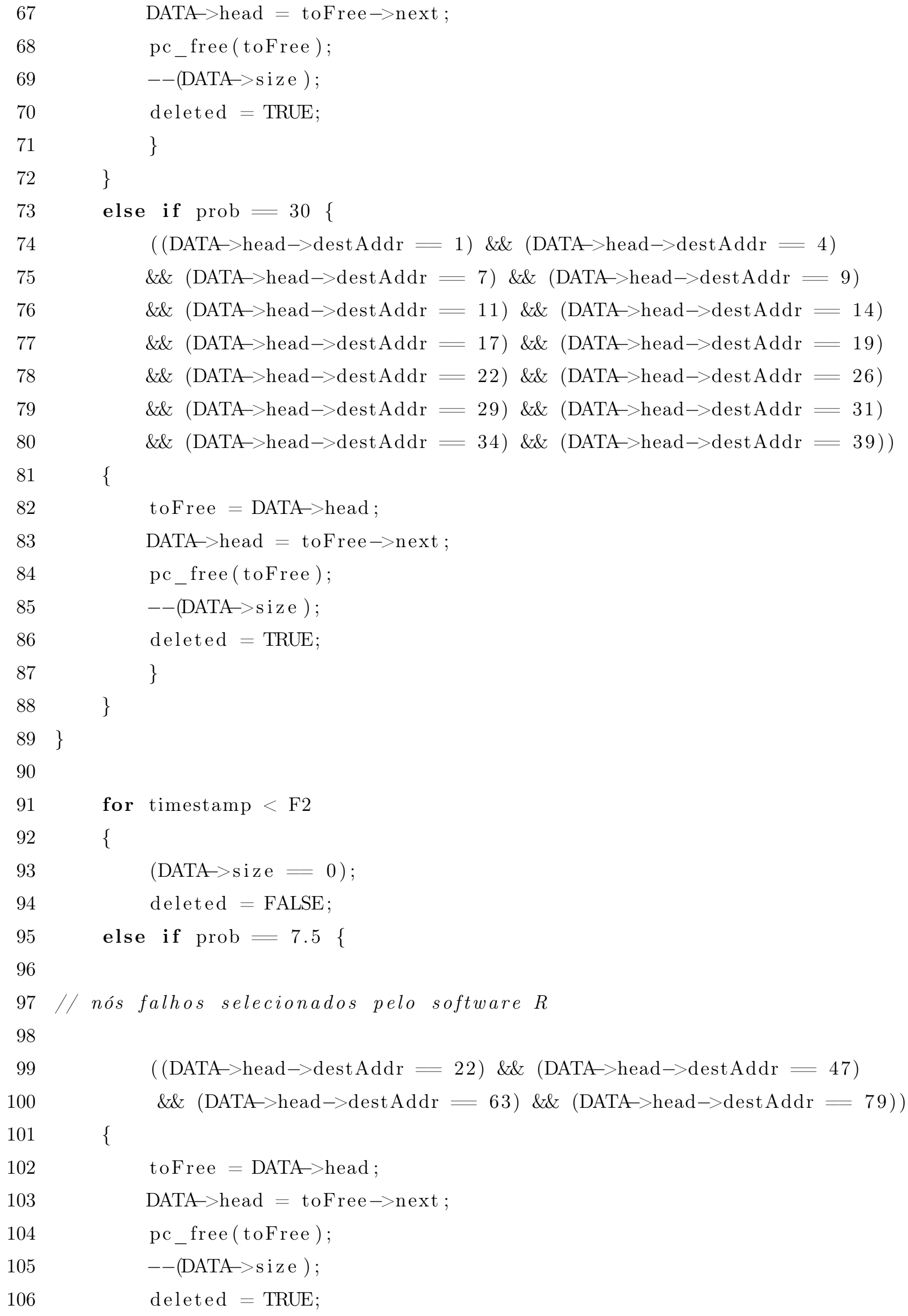




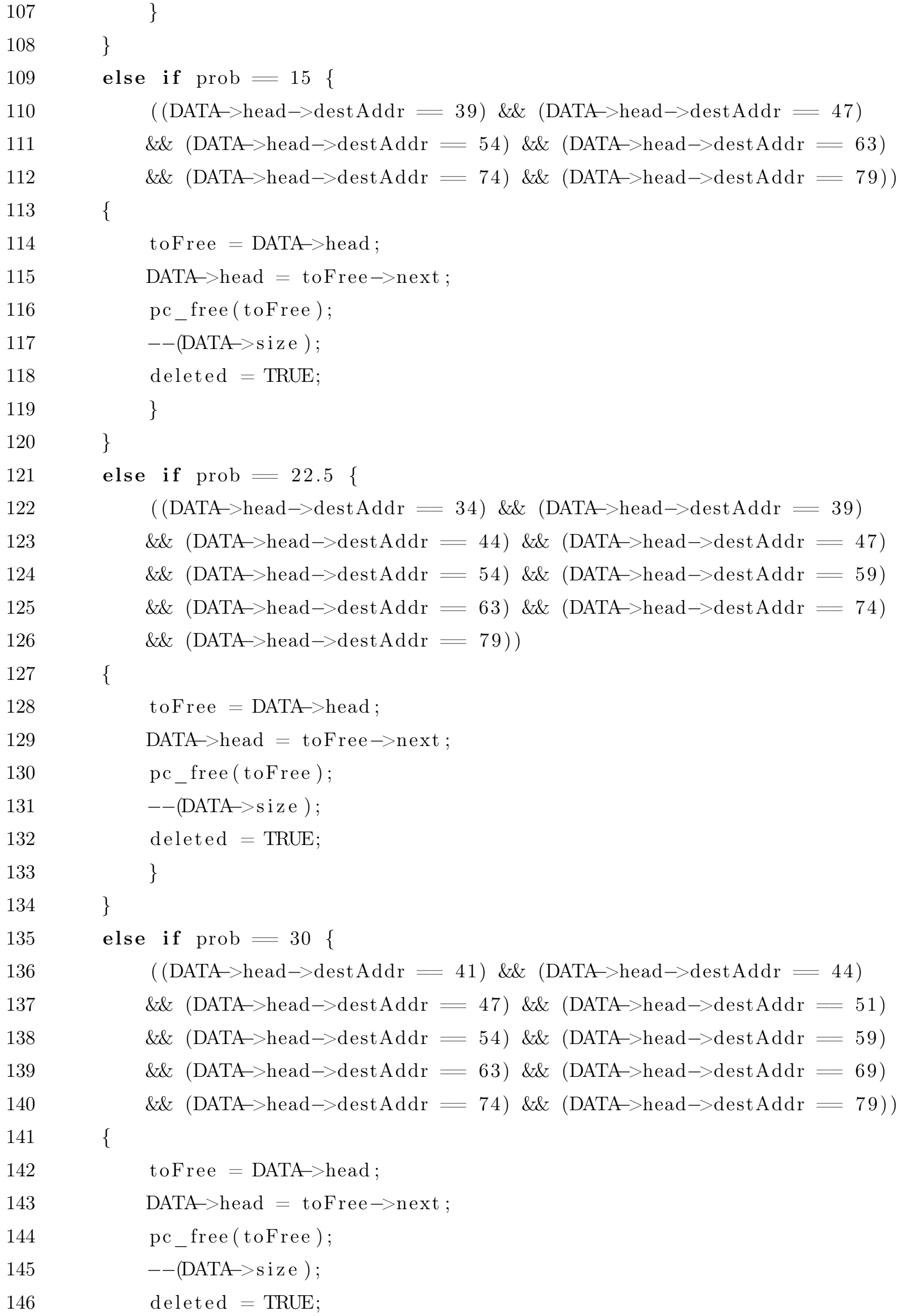




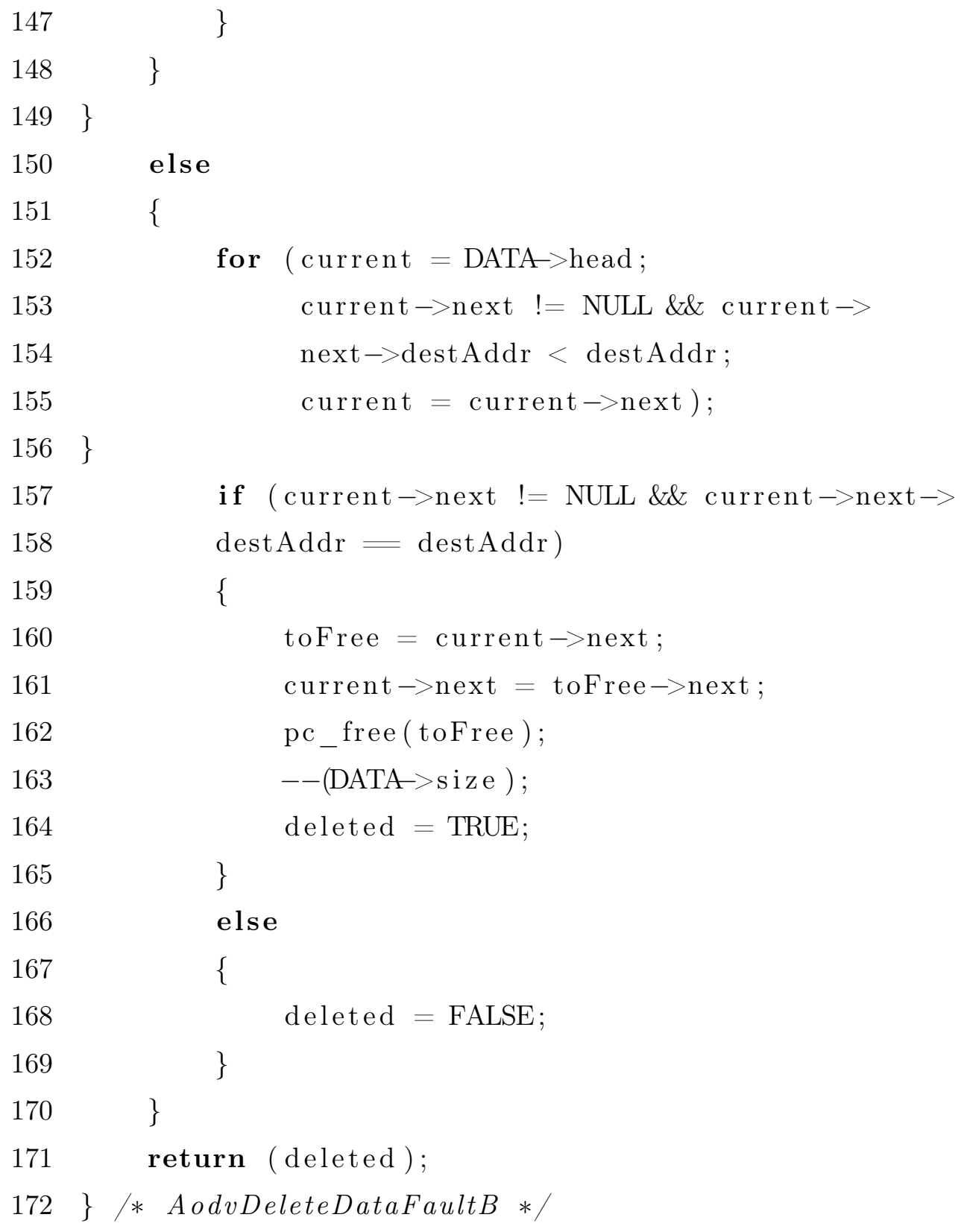




\section{Anexo F - Código fonte do algoritmo PWSave}

\section{Código original do AODV adaptado para esta dissertação}

\section{$\operatorname{aodv.h}$}

1

2 \#define estado

3

4 // função de associação ao do nó ao

5 // Quorum

6

7 void Associationnode(GlomoNode *node, Message *msg,

8 NODE_ADDR destAddr);

9

10 // função de acordar o nó

11

12 void acorda(GlomoNode $*$ node, Message $*$ msg, int ttl);

13

14

15

16 void dorme (GlomoNode $*$ node, Message $*$ msg, int ttl);

17

18 // função de associação do Quorum ao nó

19

20 void AssociacaoQuorumData(GlomoNode *node, Message *msg); 


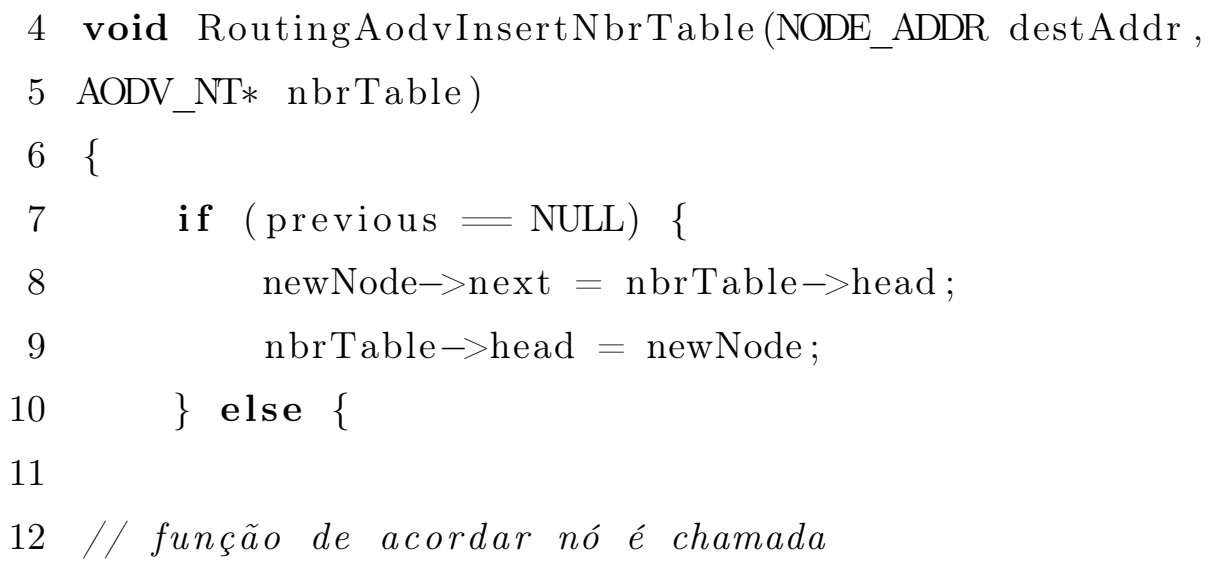
13

18 \} * RoutingAodvInsertNbrTable */

26 // Procedimento quando o RREQ é

$27 / /$ recebido

29 void RoutingAodvHandleRequest (GlomoNode *node,

30 Message $*$ msg, int $\mathrm{ttl}$ )

31\{

$33 / /$ se o nó estiver adormecido

$34 / /$ e tiver esgotado o tempo

35 // então o nó acorda 
51

52

\&aodv $\rightarrow$ seen Table ))

RoutingAodvInsertSeenTable( node, $\operatorname{rreqPkt} \rightarrow$ srcAddr, rreqPkt $\rightarrow$ bcastId, \&aodv $\rightarrow$ seen Table );

* Atualização de tabela de vizinhança

if (! RoutingAodvCheckNbrExist(rreqPkt-> lastAddr, Gaodv->nbrTable))

\{

RoutingAodvInsertNbrTable (rreqPkt->

lastAddr, Gaodv->nbrTable);

RoutingAodvIncreaseSeq(node);

\}

/ O nó é destino da rota */

if $($ node $->$ nodeAddr $=$ rreqPkt $\rightarrow$ destAddr)

\{

Routing AodvReplaceInsert RouteTable ( rreqPkt $\rightarrow$ srcAddr, rreqPkt $\rightarrow$ srcSeq, rreqPkt $\rightarrow$ hopCount, rreqPkt $\rightarrow$ lastAddr, simclock () + ACTIVE_ROUTE_TO, TRUE, TRUE, \&aodv $\rightarrow$ routeTable );

Routing AodvSetTimer ( node, MSG_NETWORK_CheckRouteTimeout, rreqPkt $\rightarrow$ srcAddr, (clocktype)ACTIVE_ROUTE_TO);

\section{* Send a Route Reply */}

Routing AodvInitiateRREP (node, msg);

\} /* if dest */

else

\{

* Não existe rota até o nó */

if (! RoutingAodvCheckRouteExist (rreqPkt $\rightarrow$

destAddr, \&aodv $\rightarrow$ routeTable)) \{

Routing AodvReplaceInsertRouteTable( rreqPkt $\rightarrow$ srcAddr, rreqPkt $\rightarrow$ srcSeq, rreqPkt $\rightarrow$ hopCount, 


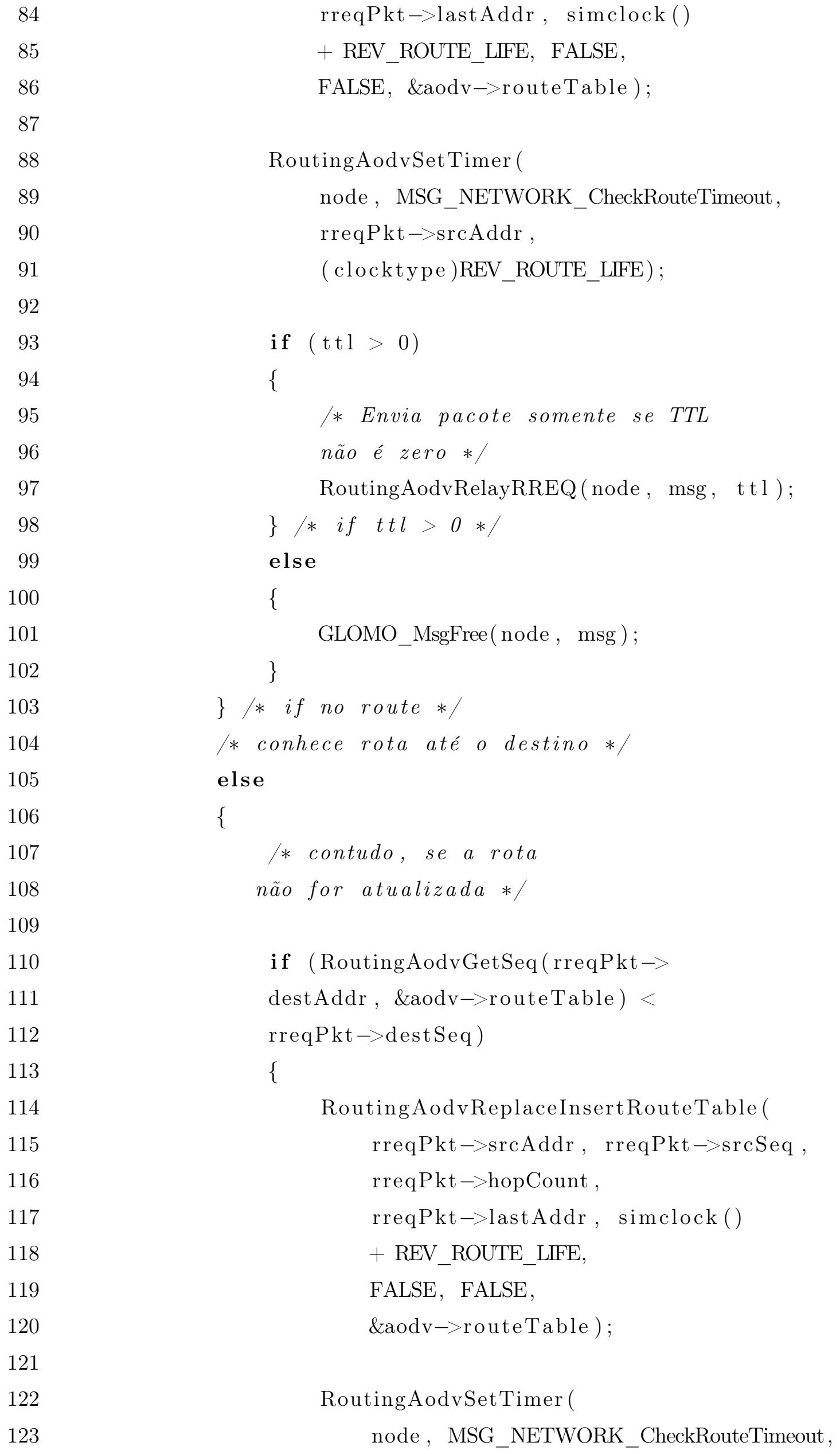


126

127

128

129

130

131

132

133

134

135

136

137

138

139

140

141

142

143

144

145

146

147

148

149

150

151

152

153

154

155

156

157

158

159

160

161

162

163
rreqPkt $\rightarrow$ srcAddr ,

( clocktype)REV_ROUTE_LIFE);

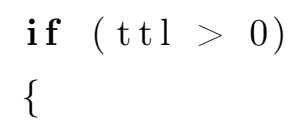

RoutingAodvRelayRREQ (node, msg, ttl);

\} $/ *$ if $t t l>0 * /$

else

\{

GLOMO_MsgFree(node, msg);

\}

\}

/* tem uma rota atualizada */

else

\{

Routing AodvReplaceInsertRouteTable ( rreqPkt $\rightarrow$ srcAddr, rreqPkt $\rightarrow$ srcSeq, rreqPkt $\rightarrow$ hopCount, rreqPkt $\rightarrow$ lastAddr, simclock ()

+ ACTIVE_ROUTE_TO,TRUE, FALSE, \&aodv $\rightarrow$ routeTable );

RoutingAodvSetTimer ( node, MSG_NETWORK_CheckRouteTimeout, rreqPkt $\rightarrow$ srcAddr, ( clocktype )ACTIVE_ROUTE_TO );

/* Envia Route Reply*/

Routing AodvInitiateRREPbyIN (node, msg);

\}$/ *$ else */

\} $/ *$ else */

\} $/$ else (nao dest) */

\} $/$ se novo pkt */

else

\{

GLOMO_MsgFree(node, msg); 


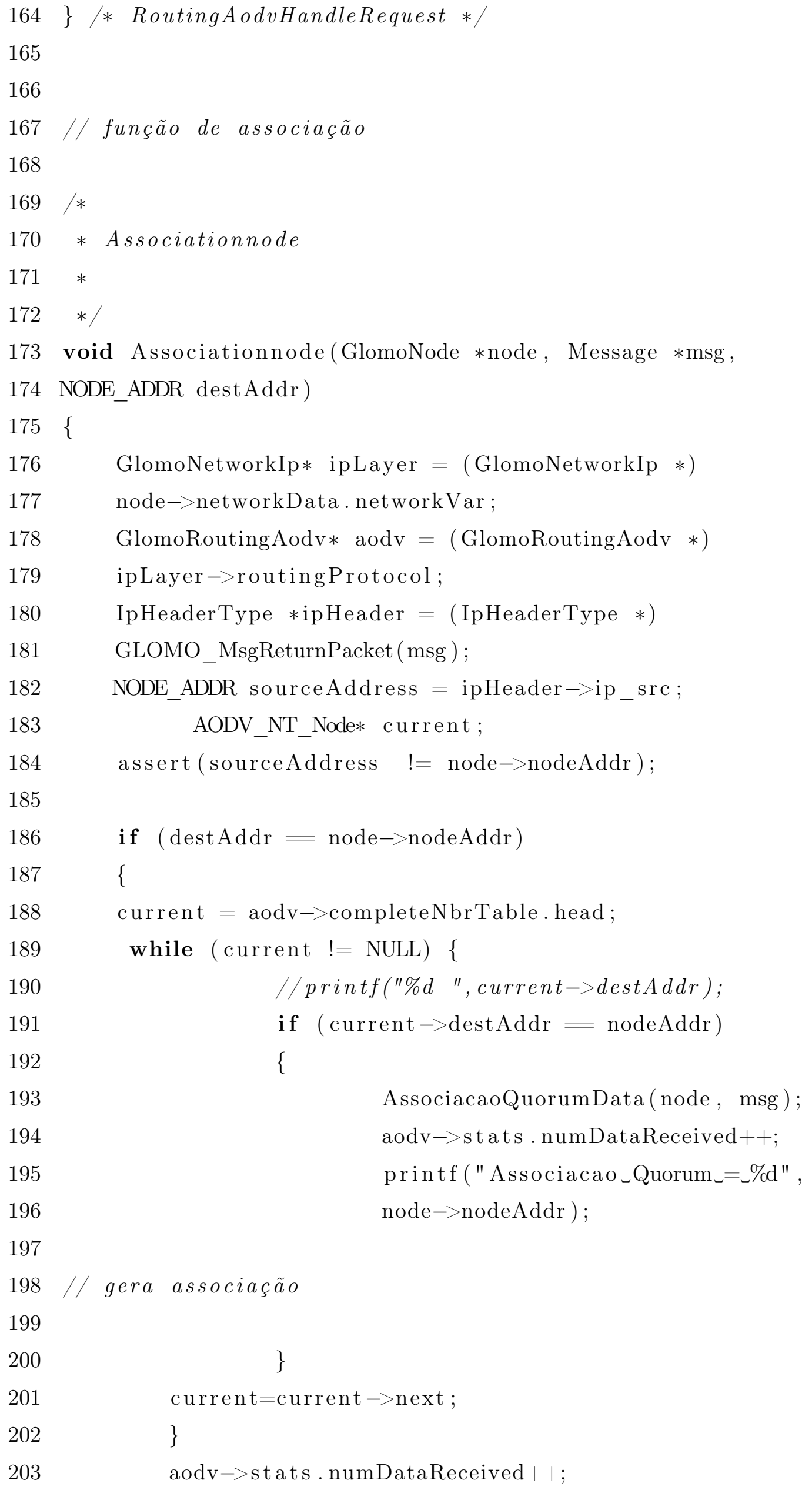

(1)


RoutingAodvUpdateLifetime (sourceAddress, \&aodv $\rightarrow$ routeTable);

RoutingAodvSetTimer(node, MSG_NETWORK_CheckRouteTimeout, sourceAddress, (clocktype)ACTIVE_ROUTE_TO);

$$
\}
$$


AODV_RREQ_Packet $*$ rreqPkt $=($ AODV_RREQ_Packet $*)$

GLOMO_MsgReturnPacket(msg);

aodv $\rightarrow$ stats . numRequestReceived ++ ;

$$
\text { * Process only if the packet is not a duplicate */ }
$$

if (! RoutingAodvLookupSeenTable (

rreqPkt $\rightarrow$ srcAddr, rreqPkt $\rightarrow$ bcastId, \&aodv $\rightarrow$ seenTable))

RoutingAodvInsertSeenTable( node, rreqPkt $\rightarrow$ srcAddr, rreqPkt $\rightarrow$ bcastId, \&aodv $\rightarrow$ seenTable );

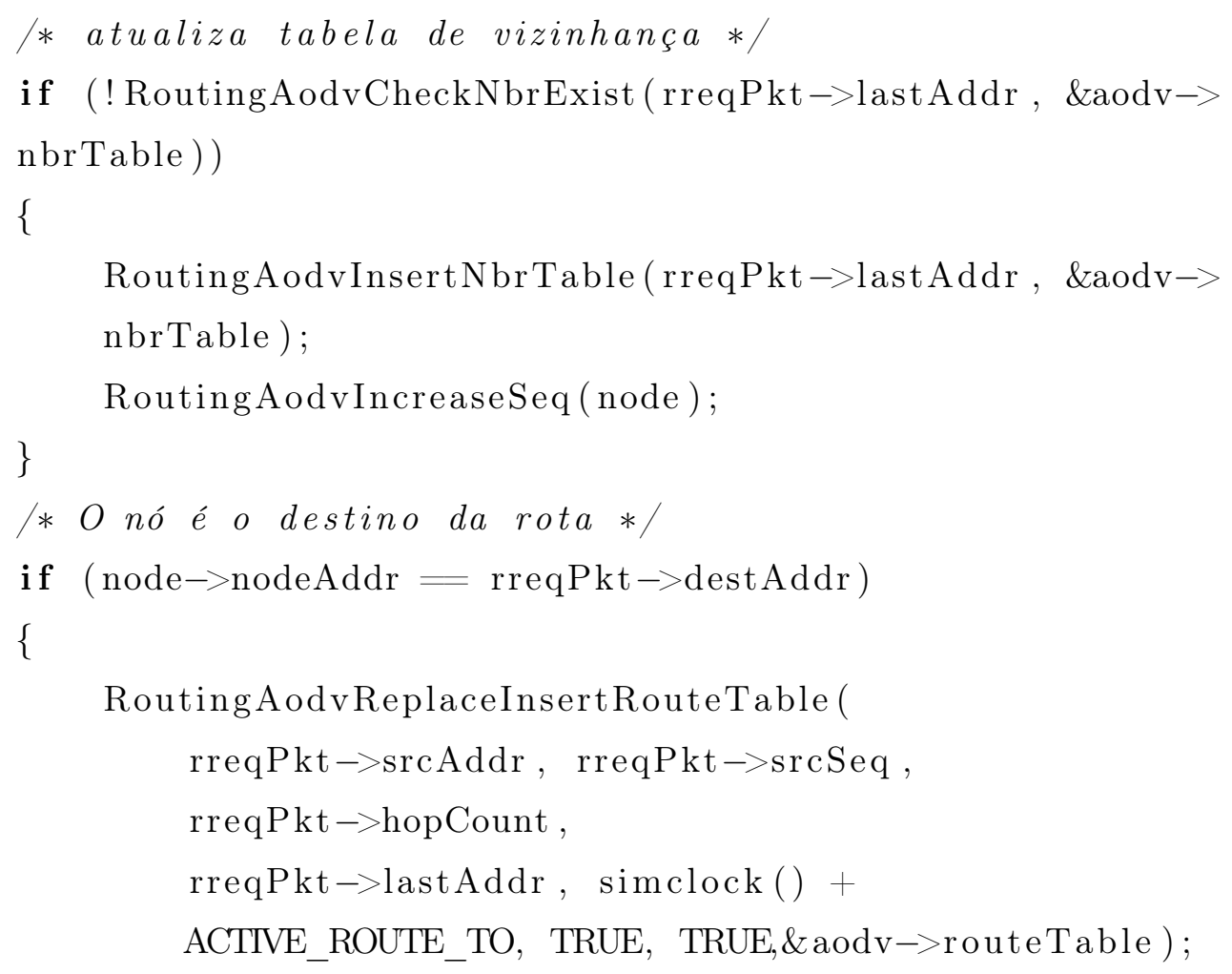


284

285

286

287

288

289

290

291

292

293

294

295

296

297

298

299

300

301

302

303

304

305

306

307

308

309

310

$311\} / * \operatorname{acorda} * /$

312

313

314

315

316

317

318 if (! RoutingAodvCheckRouteExist (rreqPkt $\rightarrow$ destAddr, \&aodv->routeTable))

\{

Routing AodvReplaceInsertRouteTable (

rreqPkt $\rightarrow$ srcAddr, rreqPkt $\rightarrow$ srcSeq ,

rreqPkt $\rightarrow$ hopCount,

rreqPkt $\rightarrow$ lastAddr, simclock ()$+$

REV_ROUTE_LIFE, FALSE,

FALSE, \&aodv $\rightarrow$ routeTable );

RoutingAodvSetTimer (

node, MSG_NETWORK_CheckRouteTimeout, rreqPkt $\rightarrow$ srcAddr

( clocktype)REV_ROUTE_LIFE); 
324

326

327

328

330

331

332

333

335

336

337

338

339

340

341

342

343

344

345

346

347

348

349

350

351

352

353

354

355

356

358

359

360

361

362

363

routingProtocol;

AODV_RREQ_Packet $*$ rreqPkt $=($ AODV_RREQ_Packet $*)$

GLOMO_MsgReturnPacket (msg);

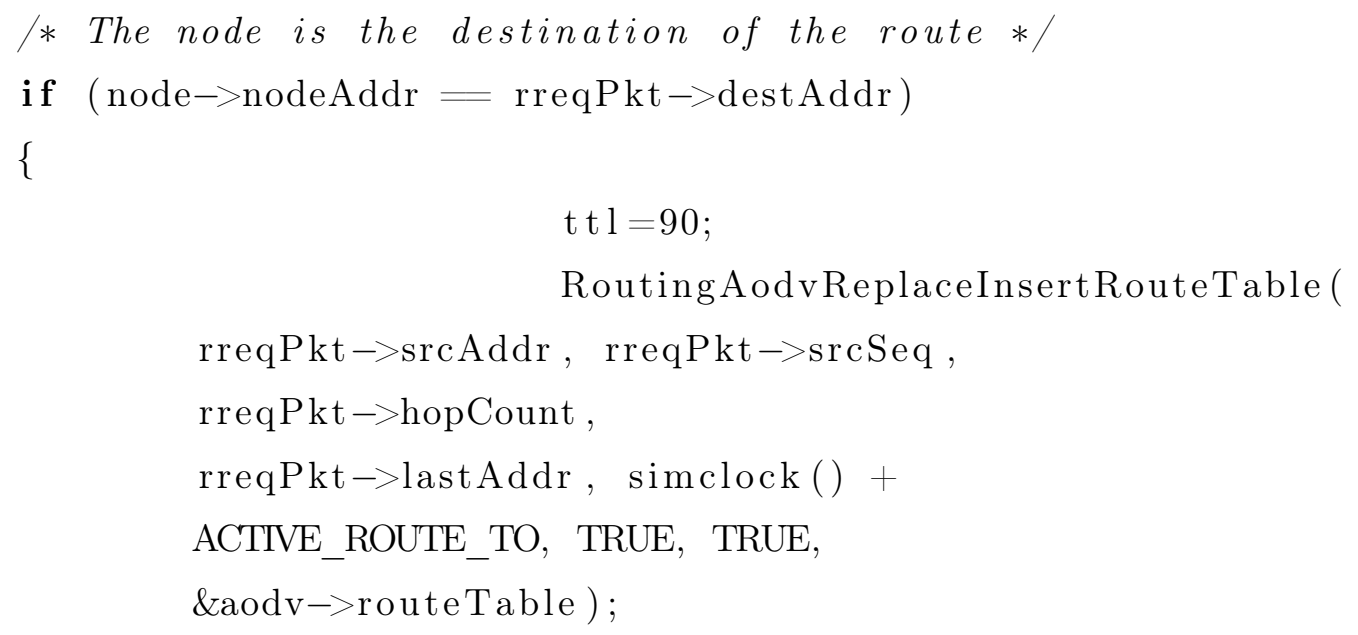

Routing AodvSetTimer (

node, MSG_NETWORK_CheckRouteTimeout, rreqPkt $\rightarrow$ srcAddr , (clocktype)ACTIVE_ROUTE_TO);

* Send a Route Reply */

RoutingAodvInitiateRREP (node, msg);

\} /* if dest */

else

\{

* checa a rota */

if (! Routing AodvCheckRouteExist (rreqPkt $\rightarrow$ destAddr, \&aodv $\rightarrow$ routeTable))

\{

Routing AodvReplaceInsertRouteTable (

rreqPkt $\rightarrow$ srcAddr, rreqPkt $\rightarrow$ srcSeq,

rreqPkt $\rightarrow$ hopCount,

rreqPkt $\rightarrow$ lastAddr, simclock () +

REV_ROUTE_LIFE, FALSE,

FALSE, \&aodv $\rightarrow$ routeTable);

RoutingAodvSetTimer (

node, MSG_NETWORK_CheckRouteTimeout, rreqPkt $\rightarrow$ srcAddr , ( clocktype)REV_ROUTE_LIFE); 


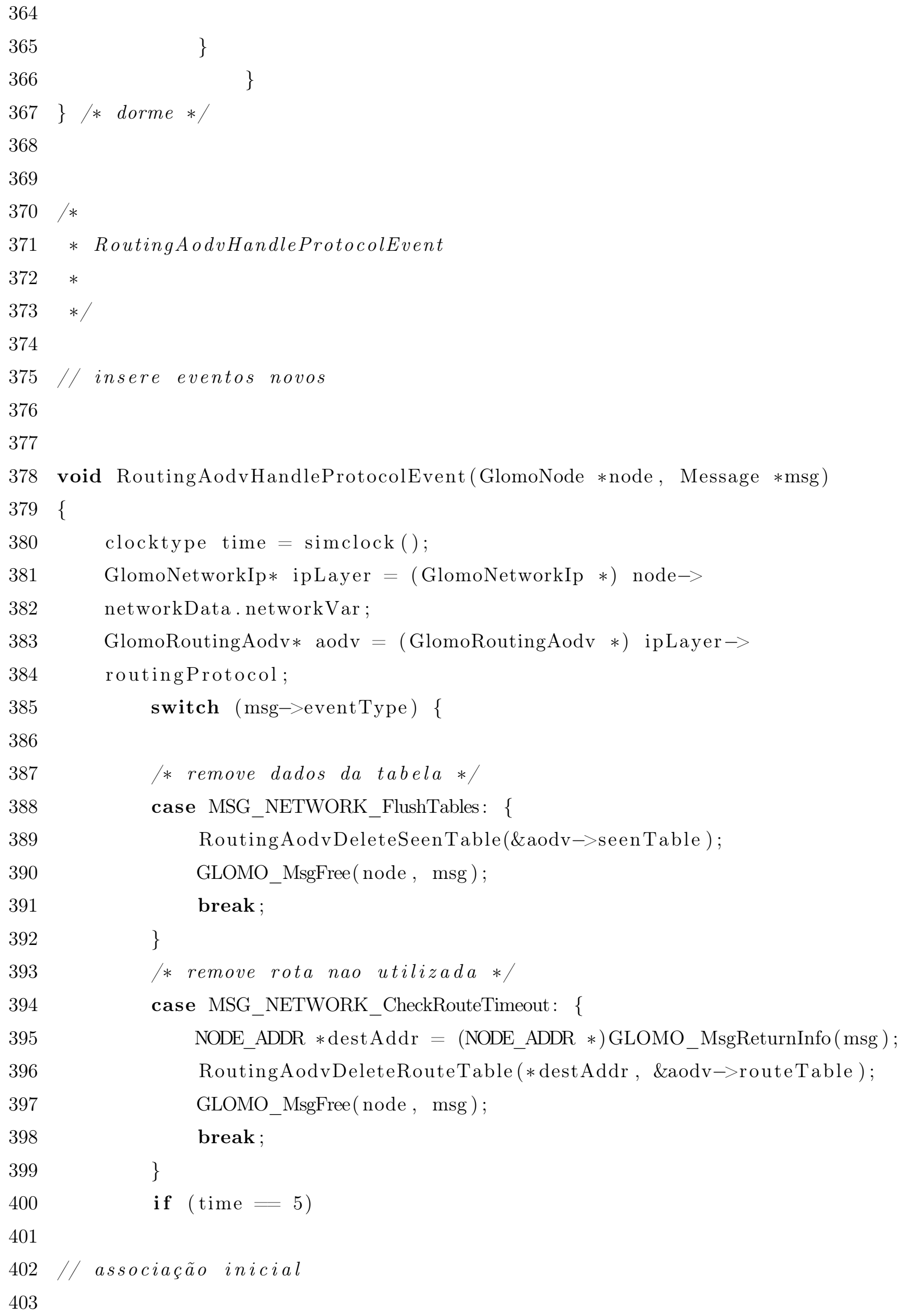




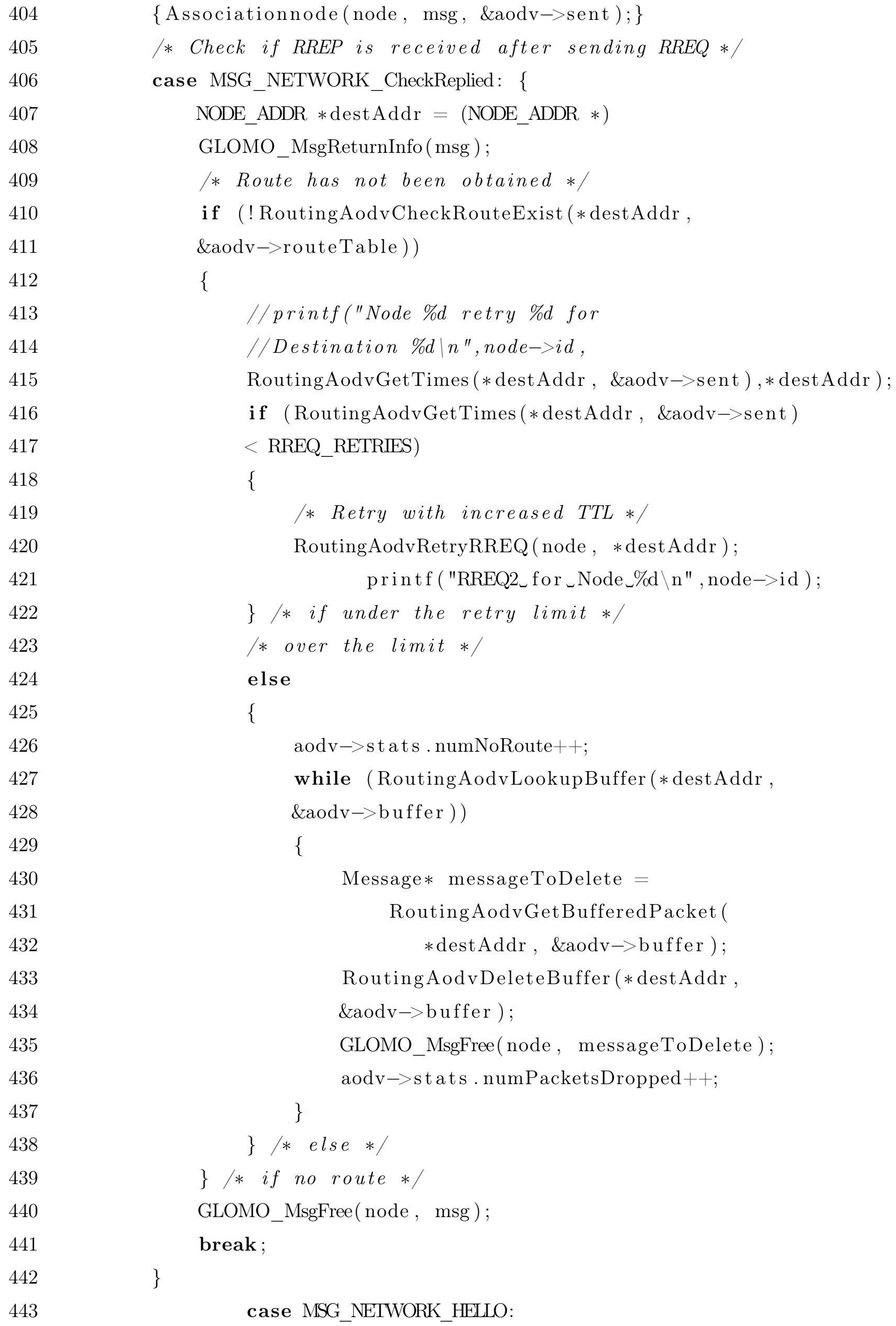

\{Associationnode(node, msg, \&aodv $\rightarrow$ sent $) ;\}$

/* Check if RREP is received after sending RREQ */

case MSG_NETWORK_CheckReplied: \{

$$
\begin{aligned}
& \text { GLOMO_MsgReturnInfo(msg); } \\
& / * \text { Route has not been obtained */ } \\
& \text { if (! RoutingAodvCheckRouteExist (*destAddr, }
\end{aligned}
$$$$
\text { NODE_ADDR } * \text { destAddr }=(\text { NODE_ADDR } *)
$$
\&aodv $\rightarrow$ routeTable))

//printf("Node \%d retry \%d for

$/ /$ Destination $\% d \mid n^{\prime \prime}$, node->id,

RoutingAodvGetTimes (*destAddr, \&aodv $\rightarrow$ sent), *destAddr);

if (RoutingAodvGetTimes (*destAddr, \&aodv $\rightarrow$ sent)

$<$ RREQ_RETRIES)

\{

/ Retry with increased TTL */

RoutingAodvRetryRREQ (node, *destAddr); printf ( "RREQ2 $\_$for $\_$Node $\_$d $\backslash \mathrm{n}$ ", node $->$ id ) ;

\} / * if under the retry limit */ /* over the limit */

else

aodv $\rightarrow$ stats . numNoRoute ++ ;

while (RoutingAodvLookupBuffer (*destAddr , \&aodv $\rightarrow$ buffer ))

\{

Message $*$ messageToDelete $=$ RoutingAodvGetBufferedPacket ( *destAddr, \&aodv $\rightarrow$ buffer ); Routing AodvDeleteBuffer (*destAddr , \&aodv $\rightarrow$ buffer ); GLOMO_MsgFree(node, messageToDelete); aodv $\rightarrow$ stats . numPacketsDropped ++ ;

\} $/ *$ else */

\} / * if no route */

GLOMO_MsgFree(node, msg);

break ;

case MSG_NETWORK_HELLO: 


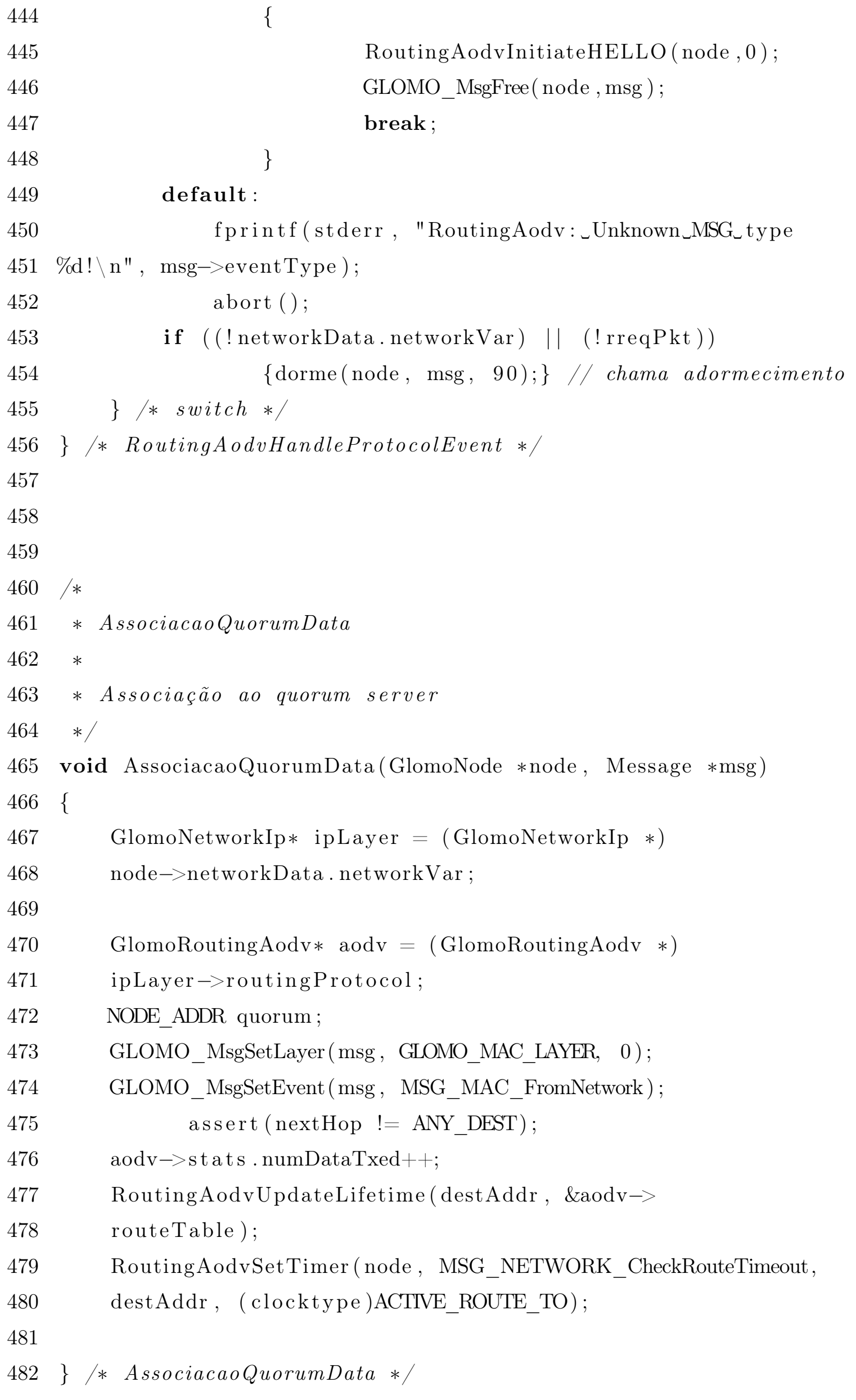

